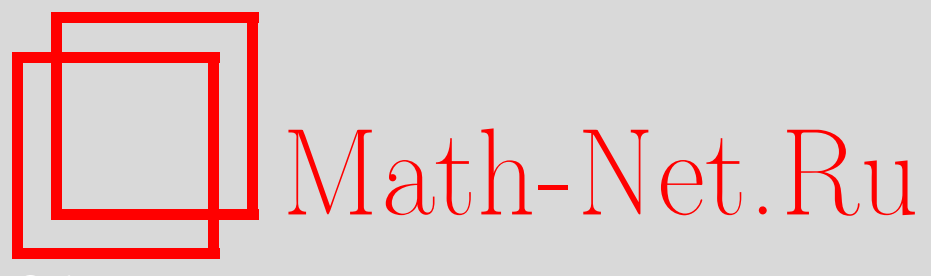

М. В. Бабич, О канонической параметризации фазовых пространств уравнений изомонодромных деформаций фуксовых систем размерности $2 \times 2$. Вывод уравнения Пенлеве VI, УМH, 2009, том 64, выпуск 1, 51-134

DOI: https://doi.org/10.4213/rm9261

Использование Общероссийского математического портала Math-Net.Ru подразумевает, что вы прочитали и согласны с пользовательским соглашением http://www . mathnet.ru/rus/agreement

Параметры загрузки:

IP : 44.207 .124 .84

26 апреля 2023 г., 13:29:55

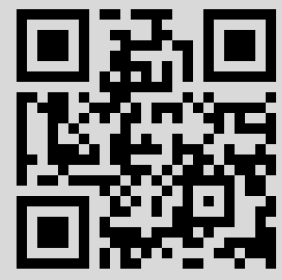




\title{
О канонической параметризации фазовых пространств уравнений изомонодромных деформаций фуксовых систем размерности $2 \times 2$. Вывод уравнения Пенлеве VI
}

\begin{abstract}
М. В. Бабич
Рассматривается факторизация, по линейным заменам искомой вектор-функции, многообразия матричных $2 \times 2$ линейных дифференциальных уравнений первого порядка с простыми полюсами в правой части. Показано, как при параметризации таких фактормногообразий естественным образом возникают уравнения Гарнье-Пенлеве VI, а также связанные с ними алгебро-геометрические конструкции - поверхность Окамото и алгебраический атлас координат Дарбу на ней.

Библиография: 42 названия.
\end{abstract}

СОДЕРЖАНИЕ

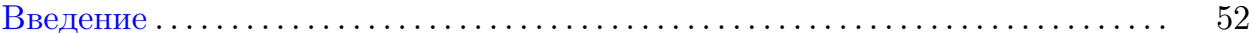

1. Системы с фуксовыми особыми точками.................... 60

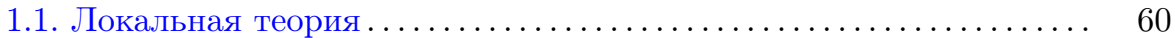

1.2. Глобальная теория, изомонодромные деформации............ 62

2. Подмногообразия изомонодромных слоев. Система Шлезингера ...... 67

3. Гамильтонов формализм и пуассонова динамика на $\operatorname{sl}(2, \mathbb{C}) \ldots \ldots \ldots \ldots .69$

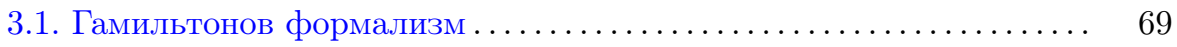

3.2. Скобка Ли-Пуассона .................................. 71

3.3. Пуассонова структура системы Шлезингера................ 74

4. Построение многообразия $M_{a_{\bar{i}}}-$ фазового пространства гамильтоновой системы Гарнье -Пенлеве VI . . . . . . . . . . . . . . . . . . . . . 77

4.1. Орбиты коприсоединенного представления $\mathrm{SL}(2, \mathbb{C}) \ldots \ldots \ldots \ldots . . \ldots 7$

4.2. Гамильтонова (симплектическая) редукция............... 80

5. Построение рациональных координат Дарбу на $M_{a_{\overline{i \imath}}} \ldots \ldots \ldots \ldots \ldots \ldots \ldots . \ldots 8$

5.1. Аналитическая геометрия в терминах $\operatorname{sl}(2, \mathbb{C}) \ldots \ldots \ldots \ldots \ldots . . \ldots 9$

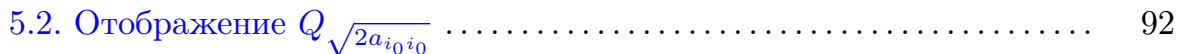

5.3. Базис, сопутствующий $A^{(i)}, A^{(j)}, A^{(k)}$; условия его существования 93

Работа выполнена при поддержке РФФИ (грант № 06-01-00451).

(C) М. В. БАБич, 2009 
5.4. Симплектичность пространства $M_{a_{\bar{i}}}$, построение сопутствую-

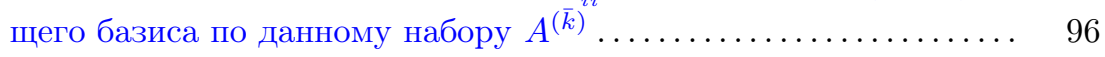

6. Система Гарнье, уравнение Пенлеве VI . . . . . . . . . . . . . . . . . 100

6.1. Рациональная (традиционная, классическая) форма $\mathrm{P}^{\mathrm{VI}} \ldots \ldots . .103$

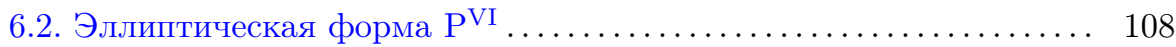

7. Поверхность начальных данных $\mathrm{P}^{\mathrm{VI}}$ (поверхность Окамото) и опреде-

ляющее многообразие ......................... 117

7.1. Симплектическая склейка квадрик - связь между координатами, построенными с помощью разных сопутствующих базисов . 117

7.2. Поверхность Окамото. Определяющее многообразие ........... 122

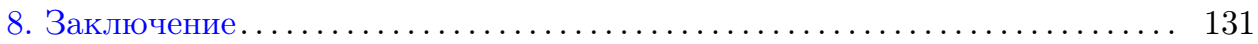

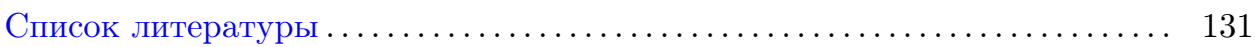

\section{Введение}

Решения уравнений класса Пенлеве претендуют на роль нелинейных специальных функций (см. [1]-[4]).

Особое место в списке этих уравнений занимает уравнение Пенлеве VI ( $\left.\mathrm{P}^{\mathrm{VI}}\right)$ наиболее общее (наименее вырожденное) из всех, и в этом смысле - наиболее фундаментальное. Выводится оно, т. е. связывается с остальным миром математики вообще и дифференциальных уравнений в частности, двумя способами - во-первых, как уравнение, решения которого обладают свойством Пенлеве, и, во-вторых, как уравнение, которое управляет коэффициентами линейного уравнения при специальных, “изомонодромных" деформациях (этого линейного уравнения). Свойство Пенлеве - специфическую зависимость общего решения от начальных данных - мы более упоминать не будем. Статья посвящена только исследованию многообразия линейных уравнений.

Получение собственно уравнения $\mathrm{PVI}$ - задача непростая как "технически", так и "идеологически". В статье решается эта и смежные с ней проблемы, в частности, строится “всё" фазовое пространство гамильтоновой системы Пенлеве VI - так называемая поверхность Окамото.

Изложение рассчитано на читателя с “формульным" (не абстрактно-алгебраическим) взглядом на математику. Предполагаемая подготовка - курс математики физического факультета университета (не обязательно Московского или Петербургского), т. е. уровень “потребителя специальных функций”.

Текст представляет собой попытку дать подробное и максимально наглядное (геометризованное) изложение связи между задачей изомонодромной деформации фуксовой системы $2 \times 2$ и уравнением $\mathrm{P}^{\mathrm{VI}}$.

Большое влияние на работу - на выбор точки зрения, материала, стиля изложения, оказали статьи [5]-[12].

Чтобы ввести в курс дела - пояснить, о чем идет речь, рассмотрим "игрушечный пример" - скалярный (коммутативный) случай:

$$
\frac{d}{d \lambda} \Psi=\sum_{k=1}^{n} \frac{A^{(k)}}{\lambda-\lambda_{k}} \Psi,
$$


здесь

$$
A^{(k)} \in \operatorname{gl}(1, \mathbb{C})=\mathbb{C}, \quad \Psi \in \mathrm{GL}(1, \mathbb{C})=\mathbb{C} \backslash\{0\}, \quad \lambda, \lambda_{k} \in \mathbb{C} .
$$

Это множество уравнений естественно рассматривать как многообразие

$$
M^{\Lambda}:=\operatorname{gl}(1, \mathbb{C})^{n} \times \mathbb{C}^{n}=\mathbb{C}^{n} \times \mathbb{C}^{n},
$$

представляющее собой декартово произведение многообразия вычетов

$$
A^{(1)}, \ldots, A^{(n)} \in \operatorname{gl}(1, \mathbb{C})^{n}=M
$$

и многообразия параметров $\lambda_{1}, \ldots, \lambda_{n} \in \mathbb{C}^{n}=\Lambda$. В основном тексте вместо $\operatorname{gl}(1, \mathbb{C})$ и $\mathrm{GL}(1, \mathbb{C})$ будут рассматриваться $\mathrm{sl}(2, \mathbb{C})$ и $\mathrm{SL}(2, \mathbb{C})$, кроме того, вместо комплексной плоскости $\mathbb{C}$ будет сфера Римана $\mathbb{C P}^{1}$ и индекс $k$, нумерующий полюсы, будет меняться не от 1 до $n$, а от 0 до $n$; будет строиться локальная по параметрам и глобальная по $\lambda$ теория, параметры $\lambda_{k}$ будут меняться каждый в своем, не пересекающемся с остальными, диске $\Lambda_{k} \subset \mathbb{C P}^{1}$.

Решение уравнения (^) можно рассматривать как многозначную функцию

$$
\Psi=c\left(\lambda-\lambda_{1}\right)^{A^{(1)}} \cdots\left(\lambda-\lambda_{n}\right)^{A^{(n)}},
$$

имеющую точки ветвления при $\lambda=\lambda_{k}, k=1, \ldots, n$. Константа $c=c\left(\lambda_{\bar{k}}\right)$ произвольно зависит от параметров.

ОБозНАчЕниЕ 1. На протяжение всего текста черта над индексом будет означать набор таких величин со всевозможными (из контекста) значениями индекса. Например, сейчас

$$
c\left(\lambda_{\bar{k}}\right)=c\left(\lambda_{1}, \ldots, \lambda_{n}\right)
$$

далее будет

$$
A^{\overline{(i)}}=A^{(0)}, A^{(1)}, \ldots, A^{(n)}
$$

и т. п.

Введем основное понятие всей теории, понятие монодромии - характеристики неоднозначности решения. Пусть переменная $\lambda$ обойдет какую-нибудь из точек $\lambda_{k}$ и вернется к своему исходному значению. Функция $\Psi$ получит не зависящий от $\lambda$ множитель $M_{k}:=e^{2 \pi i A^{(k)}} \in \mathbb{C} \backslash\{0\}$, он и называется множителем монодромии, соответствующим тому контуру, по которому $\lambda$ обошла $\lambda_{k}$.

"Набор данных монодромии" в скалярном случае - это множество чисел $e^{2 \pi i A^{(1)}}, \ldots, e^{2 \pi i A^{(n)}}$. Он локально однозначно определяет вычеты $A^{(k)}$. Оговорка "локально" необходима, потому что только при непрерывных изменениях (деформациях) число $M_{k}$ однозначно определяет $A^{(k)}=(2 \pi i)^{-1} \log M_{k}$, так как нужно фиксировать ветвь логарифма. В матричном случае тоже возможны дискретные преобразования уравнения (вычетов $A^{(k)}$ ), не меняющие набора $M_{\bar{k}}$, - это так называемые преобразования Шлезингера.

Итак, набор данных монодромии является важнейшей характеристикой линейного уравнения. Рассмотрим множество всех уравнений, обладающих одинаковыми наборами данных монодромии. Назовем изомонодромным слоем 
связную компоненту этого множества. Все пространство уравнений расслоится на такие изомонодромные слои; требуется описать это изомонодромное слоение.

В нашем "игрушечном" скалярном примере задача решается тривиально монодромии $M_{k}=\exp \left\{2 \pi i A^{(k)}\right\}$ от положения полюсов вообще не зависят, так что искомые уравнения, выделяющие $n$-мерные слои в $2 n$-мерном пространстве $\operatorname{gl}(1, \mathbb{C})^{n} \times \mathbb{C}^{n}$, неинтересны: $\partial A^{(k)} / \partial \lambda_{j}=0$ для любых $k, j$. Эта же задача в матричном случае становится нетривиальной, так как у монодромии появляется зависимость от положения полюсов $\lambda_{k}$ - изомонодромные слои “искривляются", сходят с декартова сомножителя $\mathbb{C}^{n} \ni \lambda_{1}, \ldots, \lambda_{n}$.

Рассмотрим усложнения, появляющиеся в матричном случае. Пусть в уравнении $(\star)$ вычеты $A^{(k)}$ принадлежат $\operatorname{gl}(m, \mathbb{C})$, и пусть $\Psi \in \mathrm{GL}(m, \mathbb{C})$ - какоенибудь решение (фундаментальная матрица, составленная из вектор-функций решений). Так же как и в скалярном случае, аналитическое продолжение решений по замкнутому циклу, обходящему точку $\lambda_{k}$, приводит к появлению у $\Psi$ множителя, но это уже матричный множитель: $\Psi \rightarrow \Psi M_{k} \in \mathrm{GL}(m, \mathbb{C})$. Следующим осложняющим моментом является то, что теперь монодромия не определяется уравнением - разные решения одного уравнения, если они отличаются правым множителем $\Psi(\lambda) \rightarrow \Psi(\lambda) \Phi_{\mathrm{r}}$, имеют разные наборы монодромий

$$
M_{1}, \ldots, M_{n} \rightarrow \Phi_{r}^{-1} M_{1} \Phi_{r}, \ldots, \Phi_{r}^{-1} M_{n} \Phi_{r} .
$$

Самим уравнением набор $M_{\bar{k}}$ определяется лишь с точностью до общего сопряжения. С другой стороны, теперь и функции $\Psi(\lambda)$, отличающиеся постоянным множителем (матричным, левым), удовлетворяют уже разным уравнениям домножение $\Psi(\lambda) \rightarrow \Phi_{l} \Psi(\lambda)$, т. е. линейная замена "искомой функции", приводит к преобразованию подобия коэффициентов уравнения

$$
A_{1}, \ldots, A_{n} \rightarrow \Phi_{l}^{-1} A_{1} \Phi_{l}, \ldots, \Phi_{l}^{-1} A_{n} \Phi_{l} .
$$

Возникает необходимость рассмотрения классов эквивалентности по одновременному сопряжению обоих наборов. Именно этим и создается (основная) трудность, приводящая к печально-известной громоздкости вывода уравнения Пенлеве VI. Ввести удобные координаты на фактормногообразии оказывается нелегко еще и потому, что они должны быть каноническими для некоторой гамильтоновой структуры. Структура же эта, хотя и “пришла из мира матриц", но "ничего не знает" о нашем желании рассматривать наборы и факторизовать их по общему сопряжению.

Вернемся к задаче описания изомонодромного слоя в $\operatorname{gl}(m, \mathbb{C})^{n} \times \mathbb{C}^{n}$, т. е. не проводя, пока, никаких факторизаций. Рассмотрим какую-нибудь конкретную изомонодромную деформацию $\Psi\left(\lambda ; \lambda_{1}, \ldots, \lambda_{n}\right)$, т. е. $n$-параметрическое семейство функций $\Psi(\lambda)$, каждая из которых имеет один и тот же набор монодромий $M_{1}, \ldots, M_{n}$ и только простые полюсы в $\lambda_{1}, \ldots, \lambda_{n}$ у логарифмической производной $(d \Psi / d \lambda) \Psi^{-1}$.

Такая $\Psi$ определяет $n$-мерное подмногообразие в $\left(m^{2} n+n\right)$-мерном пространстве уравнений (наборов $\left.A^{(\bar{k})}, \lambda_{\bar{k}}\right)$; назовем ее “кривой” ( $n$-мерной). Домножение справа $\Psi\left(\lambda ; \lambda_{\bar{k}}\right) \rightarrow \Psi\left(\lambda ; \lambda_{\bar{k}}\right) \Phi_{r}\left(\lambda_{\bar{k}}\right)$ будет менять монодромию, но не изменит 
этой кривой (уравнений). Домножение слева $\Psi\left(\lambda ; \lambda_{\bar{k}}\right) \rightarrow \Phi_{l}\left(\lambda_{\bar{k}}\right) \Psi\left(\lambda ; \lambda_{\bar{k}}\right)$ будет изгибать эту кривую, но так, что она не будет сходить с интересующего нас изомонодромного слоя (домножение слева не меняет монодромий).

При всевозможных таких изгибах каждая точка кривой, т. е. набор вычетов $A^{(1)}, \ldots, A^{(n)}$, превратится в $\left(m^{2}-1\right)$-мерное ${ }^{1}$ многообразие, в орбиту

$$
\bigcup_{g \in \operatorname{GL}(m, \mathbb{C})} g^{-1} A^{(1)} g, \ldots, g^{-1} A^{(n)} g .
$$

Интересующий нас изомонодромный слой окажется $\left(m^{2}-1+n\right)$-мерной поверхностью в $\left(m^{2} n+n\right)$-мерном пространстве, заметенной $n$-мерными “кривыми" при калибровочных “изгибах" $\Phi_{l}\left(\lambda_{\bar{k}}\right)$.

Предлагается следующая иллюстрация. Евклидово трехмерное пространство (это аналог пространства уравнений) некоторым условием (условием изомонодромности) наслоено на поверхности (изомонодромные слои), переходящие в себя при вращении вокруг оси $O Z$ (это действие калибровочной группы $\mathrm{GL}(m, \mathbb{C})$ на $\Psi$-функцию слева). Роль параметров деформации $\lambda_{\bar{k}}$ в этом примере играет координата на оси $O Z$. Иными словами, пространство некоторым специальным (искомым) образом наслоено на поверхности вращения вокруг оси $O Z$. Любая кривая, лежащая на какой-нибудь из этих поверхностей вращения, будет обладать нужным свойством (ее точки-уравнения имеют одинаковые наборы монодромий), причем через любую точку можно провести бесконечно много разнообразных кривых (размерность слоя $m^{2}-1+n$ больше $n$ - числа параметров $\left.\lambda_{1}, \ldots, \lambda_{n}\right)$. В данной статье решается задача описания этих слоевискомых поверхностей вращения.

Естественным способом описания поверхности вращения является задание какой-нибудь направляющей, один раз пересекающей все окружности (орбиты действия калибровочной группы $\mathrm{GL}(m, \mathbb{C}))$. Можно, например, задать направляющую плоской кривой в пространстве - пересечением поверхности вращения плоскостью, содержащей ось, - тогда в уравнениях движения точки трехмерного пространства по этой кривой не будет участвовать угловая переменная, и сразу после введения координат на кривой эти уравнения можно интерпретировать как искомые уравнения изомонодромных деформаций (уравнения Гарнье-Пенлеве VI). Эта плоская направляющая кривая является (задается) решением системы уравнений Шлезингера, которой удовлетворяют изомонодромные деформации $\Psi\left(\lambda ; \lambda_{\bar{k}}\right)$, обладающие дополнительным (наложенным "руками") свойством:

$$
\lim _{\lambda \rightarrow \infty} \Psi\left(\lambda ; \lambda_{\bar{k}}\right)=\text { const } \in \operatorname{GL}(m, \mathbb{C}) \quad \forall \lambda_{k} .
$$

Обычно точки этой же линии (решение уравнений Шлезингера) и используют для параметризации "образующих окружностей” (введения координат на фактормногообразии по действию калибровочной группы). Этот путь известен весьма тяжеловесными вычислениями, не освещенными какой бы то ни было

\footnotetext{
${ }^{1}$ Размерность не $m^{2}$, а $m^{2}-1$, на единицу меньше размерности $\operatorname{GL}(m, \mathbb{C})$, так как пропорциональные матрицы одинаково преобразуют наборы $A^{(\bar{k})}$.
} 
геометрической моделью (см. [1]); кроме того, рассуждения для общего положения при некоторых наборах параметров не работают, необходимо рассматривать различные случаи. Заметим в этой связи, что аналитическая сложность всей теории связана еще и с тем, что мы изначально имеем дело с многообразиями очень высоких размерностей. Например, для уравнения Пенлеве $n=3$, $m=2$, так что размерность пространства уравнений $m^{2} n+n=15$ (!), в то время как истинная размерность задачи равна трем (!) - именно трехмерное расширенное фазовое пространство оказывается наслоенным на одномерные подмногообразия, решения уравнения Пенлеве VI. Для такого уменьшения размерности приходится прибегать к множеству ухищрений, затуманивающих нарисованную выше схематическую картину, - переходить от группы GL к SL и от алгебры gl к sl (деление на скаляр $\Psi \rightarrow \Psi / \sqrt{\operatorname{det} \Psi}$ не меняет локального постоянства монодромий), к подмногообразиям матриц с фиксированными собственными числами, факторизовать по действию калибровочной группы и т. д.

Заметим здесь, что изрядную часть этой работы можно было бы опустить отнести на счет общей теории, - перейдя на более адекватный, но более абстрактный язык теории связностей в расслоениях. Именно на этом языке вся теория выглядит наиболее естественной, на нем были получены наиболее тонкие результаты теории изомонодромных деформаций, решена 21-я проблема Гильберта [13], [14].

K сожалению, как и в художественной литературе, смена языка означает смену читательской аудитории, поэтому мы не будем давать определений и сколько-то последовательного изложения, а лишь на словах поясним, о чем идет речь - что такое векторное расслоение со связностью, в чем сходство и различие изомонодромных деформаций уравнений и связностей (подробнее см. [14], [15]).

Расслоение - это многообразие, которое снабжено некоторой дополнительной структурой. В нашем случае систем $2 \times 2$ линейных уравнений на $\mathbb{C P}^{1}$ нужны расслоения с базой $\mathbb{C P}^{1}$, слоем $\mathbb{C}^{2}$ и структурной группой $\mathrm{GL}(2, \mathbb{C})$. Этот случай и будем рассматривать.

Пространство расслоения $E$ - это трехмерное многообразие, на котором задана проекция на базу $\mathrm{pr}: E \rightarrow \mathbb{C P}^{1}$. Прообраз $\mathrm{pr}^{-1}(\lambda)$ каждой точки $\lambda \in \mathbb{C P}^{1}$ диффеоморфен слою $\mathbb{C}^{2}$. Структура расслоения "склеивает" эти, изначально никак не связанные, слои-прообразы в трехмерное многообразие $E$ : база покрывается окрестностями $\bigcup_{\alpha} D_{\alpha}=\mathbb{C P}^{1}$, и в каждом прообразе $\operatorname{pr}^{-1}\left(D_{\alpha}\right) \subset E$ вводятся координаты прямого произведения $\phi_{\alpha}: \operatorname{pr}^{-1}\left(D_{\alpha}\right) \rightarrow D_{\alpha} \times \mathbb{C}^{2}$ так, чтобы возникающие на перекрытиях $D_{\alpha} \cap D_{\beta} \neq \varnothing$ автоморфизмы кроме стандартных условий (коцикла), обеспечивающих корректность построения $E$ как многообразия, еще имели дополнительные свойства:

- естественная проекция $\operatorname{pr}_{\alpha}: D_{\alpha} \times \mathbb{C}^{2} \rightarrow D_{\alpha}$ на первый декартов сомножитель согласована с проекцией $\mathrm{pr}: \mathrm{pr}=\mathrm{pr}_{\alpha} \circ \phi_{\alpha}$, т. е. слой отображается в слой;

- индуцированные в пересечениях $D_{\alpha} \cap D_{\beta}$ автоморфизмы слоев $\mathbb{C}^{2} \rightarrow \mathbb{C}^{2}$ принадлежат структурной группе $\mathrm{GL}(2, \mathbb{C})$. 
Связностъю в расслоении называется отображение, которое каждому пути $l:[0,1] \rightarrow \mathbb{C P}^{1}$ на базе ставит отображение между слоями над конечной и начальной точками, принадлежащее структурной группе. При этом кроме непрерывности и независимости от параметризации должно быть выполнено условие аддитивности: если какая-нибудь кривая разбита на две так, что конец одной совпадает с началом другой, то соответствующее этой составной кривой отображение слоев должно совпадать с композицией отображений, определенных ее частями.

После введения локальных координат связность может быть задана системой дифференциальных уравнений:

$$
d \Psi(\lambda)=\omega(\lambda) \Psi(\lambda)
$$

Здесь $\Psi=\left(\psi_{1}, \psi_{2}\right)^{T}$ - координаты в слое над точкой базы $\lambda$. Координаты эти определены базисом $\left(\vec{e}_{1}, \vec{e}_{2}\right)=\left(\vec{e}_{1}, \vec{e}_{2}\right)(\lambda)$, выбранным в слое при введении координат (сама точка слоя - это $\left.\left(\vec{e}_{1}, \vec{e}_{2}\right) \Psi\right)$.

Эта система уравнений, конечно, определена толъко там, где определены эти локальные координаты. Если координаты прямого произведения на расслоении можно ввести глобально, то такое расслоение называется тривиальным. Именно тривиальные расслоения эквивалентны системам уравнений, заданным на базе.

Матричнозначная $(2 \times 2)$-форма

$$
\omega=\left(\begin{array}{ll}
\omega_{11} d \lambda & \omega_{12} d \lambda \\
\omega_{21} d \lambda & \omega_{22} d \lambda
\end{array}\right), \quad \omega_{i j}=\omega_{i j}(\lambda),
$$

называется формой связности. Она, в координатах $\left(\vec{e}_{1}, \vec{e}_{2}\right)$, задает преобразование слоя над $\lambda$ в слой над $\lambda+d \lambda$ при инфинитезимальном сдвиге $d \lambda$ из точки $\lambda$.

В зависимости от свойств $\omega_{i j}(\lambda)$ различают гладкие, аналитические, мероморфные и прочие связности. Для фуксовых систем $\omega_{i j}(\lambda)$ - это мероморфные формы на $\mathbb{C P}^{1}$ с только простыми полюсами.

Замена базиса $\left(\vec{e}_{1}, \vec{e}_{2}\right)=\left(\vec{e}_{1}^{\prime}, \vec{e}_{2}^{\prime}\right) \Phi$ в слое над каждой точкой $\lambda$ приводит к замене (искомой, зависимой) переменной - домножению $\Psi=\Psi(\lambda)$ слева на $\Phi=\Phi(\lambda)$, т. е. к калибровочному преобразованию уравнения:

$$
d \Psi^{\prime}=\left(d \Phi \Phi^{-1}+\Phi \omega \Phi^{-1}\right) \Psi^{\prime}
$$

Таким образом, исследуя связность, мы сразу исследуем целый класс уравнений, связанных линейными заменами искомой функции.

Понятия монодромии и изомонодромной деформации естественно формулируются на этом языке. Монодромия связности - это автоморфизм слоя, задаваемый замкнутой кривой на базе. При изомонодромных деформациях эти автоморфизмы не должны меняться.

Итак, на языке теории связностей в расслоениях, основная часть данного обзора посвящена специальному выбору базисов $\left(\vec{e}_{1}, \vec{e}_{2}\right)$ в слоях тривиального расслоения над $\mathbb{C P}^{1}$. 
Вернемся к дифференциальным уравнениям. В работах японской школы [9], [10], [16] была продемонстрирована эффективность методов теории гамильтоновых систем в задачах изомонодромной деформации. С этой точки зрения исследуемые фуксовы системы являются точками фазового пространства, а их изомонодромные деформации - это траектории соответствующей гамильтоновой системы.

То, что координатами Дарбу для многих гамильтоновых систем, имеющих представление нулевой кривизны, являются положения полюсов блоховской функции и собственные значения $L$-оператора в этих точках, впервые установлено в работе [17] и, в значительно более общем виде, в [18] (приложение к теории Виттена-Зайберга см. [19], [20]). В конкретном случае фуксовых систем, отвечающих уравнению Пенлеве VI, эти координаты представляют собой пару функций $p, q$ - положение корня антидиагонального члена правой части уравнения $(\star)$ и значение диагонального члена в этой точке:

$$
q:\left(\sum_{k=1}^{3} \frac{A^{(k)}}{q-\lambda_{k}}\right)_{12}=0, \quad p=\left(\sum_{k=1}^{3} \frac{A^{(k)}}{q-\lambda_{k}}\right)_{11} .
$$

Тут представитель семейства калибровочно-эквивалентных друг другу уравнений выбирается так, чтобы вычет $A^{(0)}$ в бесконечно удаленной точке $\lambda_{0}=\infty$ был диагонален, такую калибровку будем называть традиционной.

В данной статье предлагается другая калибровка, ее можно было бы назвать треугольной. Прежде всего она отличается тем, что для выбора уравненияпредставителя используется не одна (это была $\lambda_{0}=\infty$ ), а три различных точки, в тексте они обозначены $\infty_{+}, \infty_{-}$и $\infty_{\star}$ (см. с. 68$)$.

Вообще идея треугольной нормировки функции $\Psi$ одновременно в разных точках не нова. В работе [21] И. М. Кричевер использовал аналогичную нормировку решения вспомогательной линейной системы при исследовании двухточечной функции Бейкера-Ахиезера. В окрестности точки $\lambda=0$ матрица $\Psi$ выбиралась верхнетреугольной, с единицами на диагонали, а в окрестности точки $\lambda=\infty$ матрица $\Psi$ была нижнетреугольной. Предлагаемая в данной статье нормировка (16), кроме наличия третьей нормировочной точки, от нормировки И. М. Кричевера отличается тем, что треугольный вид имеет не сама функция $\Psi$, т. е. не решение линейной системы, а логарифмическая производная $\left(\frac{\partial}{\partial \lambda} \Psi\right) \Psi^{-1}$ или, в точке ветвления $\Psi$, вычет этой логарифмической производной. Это более слабое, чем треугольность, ограничение на $\Psi$, оно эквивалентно условию линейной зависимости между строкой и ее производной.

Нормированная таким образом система обладает замечательным свойством: если для калибровки использовать, скажем, вычеты $A^{(0)}, A^{(2)}$ и $A^{(3)}$, то естественная проекция подмногообразия уравнений на орбиту $\mathscr{O}$, заметаемую вычетом $A^{(1)}$ :

$$
A^{(0)}, A^{(1)}, A^{(2)}, A^{(3)} \rightarrow A^{(1)},
$$

окажется каноническим отображением. Соответственно любая пара канонически-сопряженных переменных $p, q$ на орбите $\mathscr{O}$ - стандартном симплектическом пространстве - даст каноническую параметризацию многообразия уравнений. 
Несколько слов по поводу общности наших построений.

Имеется подсемейство уравнений ( $)$, все вычеты которых могут быть одновременно приведены к верхнетреугольному виду. Такие наборы вычетов мы назовем одновременно-треугольными. "Одновременно-треугольный” случай много проще общего и не нуждается в теории, изложению которой посвящена эта статья.

Действительно, приведем набор $A^{(\bar{k})}$ к верхнетреугольному виду $A_{21}^{(k)}=0$ для любого $k$; поскольку собственные числа $A_{11}^{(k)}$ вычетов фиксируются монодромиями, то соответствующие наборы параметризуются просто отношением $A_{12}^{(0)}: A_{12}^{(1)}: \cdots: A_{12}^{(n)}-$ точками проективного пространства. Соответствующее изомонодромное подмногообразие будет задаваться системой линейных уравнений на $A_{12}^{(k)}$; в случае Пенлеве $n=3$ эта система сводится к гипергеометрическому уравнению.

УСловие. Мы исключаем из рассмотрения случай, когда все матрицы $A^{(i)}$, $i=0, \ldots, n$, могут быть одновременно приведены $\kappa$ верхнетреугольному виду.

Назовем это условием одновременной нетреугольности. Заметим, что если компонента такая существует, то из необходимости равенства нулю суммы всех вычетов $\sum A^{(k)}$ следует, что сумма параметров - собственных чисел - равна нулю, т. е. "одновременно-треугольная" компонента существует только при специальных значениях параметров. Предложенный способ параметризации (линейных уравнений) позволяет единообразно рассматривать всё пространство линейных уравнений, кроме одновременно-треугольного его подмногообразия. Итак, это одновременно-треугольное множество удалено из всех рассматриваемых многообразий.

На параметры исследуемого изомонодромного слоя (параметры Пенлеве $\alpha$, $\beta, \gamma, \delta)$, иначе - на собственные числа вычетов $A^{(k)}$, не накладывается никаких ограничений.

Отметим теперь следующий тонкий момент. Множество уравнений с одинаковыми монодромиями устроено не просто. Прежде всего, имеется компонента, существующая при всех значениях параметров (собственных чисел матриц-вычетов $\left.A^{(k)}\right)$. Эту компоненту можно назвать регулярной или илезингеровой. Именно она описывается уравнением Пенлеве VI, и вычеты $A^{(k)}$, не покидающие эту компоненту при деформациях, удовлетворяют (при надлежащей калибровке) системе уравнений Шлезингера (15). Данная статья посвящена описанию именно этой - шлезингеровой - компоненты.

В случае общего положения - отсутствия натуральных разностей между собственными числами $A^{(k)}$ - этой компонентой все и исчерпывается. Однако при некоторых значениях параметров имеются и другие семейства уравнений с совпадающими монодромиями, осложняющие картину и являющиеся причиной множества оговорок при формулировках. Эти сложные случаи обычно называют резонансами. Случай резонансов является предметом интенсивных исследований [22], [14], [23], однако лежит далеко за пределами данной статьи. Мы будем исследовать толъко деформации, описываемые уравнением Пенлеве VI. 
В заключение, однако, заметим, что хотя уравнения, описывающие резонансные изомонодромные деформации, в статье и не обсуждаются, но сами точки этих исключительных (резонансных) семейств автоматически оказываются параметризованными (кроме уже оговоренных одновременно-треугольных) вместе со всеми остальными точками $M_{a_{\bar{i}}}$.

Возвращаясь к системе Шлезингера, укажем, что она потребуется нам только для получения уравнений изомонодромных деформаций. Параметризовать же орбиты (вводить необходимые для дальнейшего исследования канонические координаты) предлагается другим образом (см. [24]-[26]), ему соответствует другая “направляющая", заданная уравнениями (13), (14), где $R$ приведено в (17), но в дальнейшем эти уравнения не потребуются. В результате естественным образом появляется многообразие $M_{a_{\bar{i}}}$ - фазовое пространство гамильтоновой системы, задающей листы изомонодромного слоения (системы Гарнье-Пенлеве VI); $M_{a_{\overline{i \imath}}}$ строится как симплектическое алгебраическое многообразие - объединение аффинных квадрик (орбит коприсоединенного представления $\mathrm{SL}(2, \mathbb{C}))$ с алгебраическими (даже бирациональными) функциями переклейки, сохраняющими симплектическую структуру. Это многообразие является частью так называемой поверхности Окамото, или поверхности начальных данных уравнения $\mathrm{P}^{\mathrm{VI}}$ (см. [16], [8]). Для построения всей поверхности Окамото к $M_{a_{\overline{i i}}}$ нужно будет добавить (см. раздел 7) еще одну линию (дивизор, см. [27]), точки которой соответствуют решениям, обратившимся в этот момент в бесконечность.

Венцом-итогом развиваемой теории является доказательство теоремы, принадлежащей К. Окамото и утверждающей, что на соответствующем расширенном фазовом пространстве нет другого поля направлений (алгебраического, т. е. направляющие косинусы которого были бы алгебраически связаны с координатами на многообразии), кроме как задаваемого уравнением Пенлеве VI. Теорема эта интересна тем, что позволяет свести исследование важного дифференциального уравнения к изучению геометрии некоторого алгебраического многообразия.

\section{1. Системы с фуксовыми особыми точками}

Объектом нашего исследования является семейство (матричных) линейных дифференциальных уравнений

$$
\frac{d}{d \lambda} \Psi=\sum_{k=0}^{n} \frac{A^{(k)}}{\lambda-\lambda_{k}} \Psi, \quad \Psi \in \mathrm{SL}(2, \mathbb{C}), \quad A^{(k)} \in \mathrm{sl}(2, \mathbb{C}) .
$$

Системы такого вида, т. е. с полюсами только первого порядка, называются фуксовыми (в конце ХIX века их исследовал Л. Фукс [28], современные изложения см. в [1], [3], [4]).

1.1. Локальная теория. Данная статья посвящена исследованию деформаций такого уравнения, т. е. будет изучаться зависимость коэффициентов $A^{(k)}$ и решения $\Psi$ от параметров $\lambda_{0}, \lambda_{1}, \ldots, \lambda_{n}$, но пока пусть все параметры $\lambda_{k} \oint u \kappa$ cuрованъ. Как ведет себя $\Psi(\lambda)$ в окрестности особой точки $\lambda=\lambda_{k}$ ? 
Введем координату

$$
x:=\lambda-\lambda_{k} .
$$

Локальные исследования уравнения

$$
\frac{d}{d x} \Psi=\left(\frac{A}{x}+\mathbf{O}(1)\right) \Psi,
$$

где $\mathbf{O}(1)$ аналитична в некоторой окрестности нуля, показывают (см. [29]), что решение $\Psi$ может быть представлено в виде

$$
\Psi=\Psi_{\mathrm{reg}}(x) \Psi_{\mathrm{sing}}(x) \Psi_{\mathrm{mdr}},
$$

где $\Psi_{\text {reg }}(x)$ регулярна (в частности - обратима), $\Psi_{\mathrm{mdr}}$ постоянна (не зависит от $x)$, а $\Psi_{\text {sing }}(x)$ имеет некоторый стандартный вид. Именно, $\Psi_{\text {sing }}$ является негладкой и обеспечивает особенность (простой полюс) у $d \Psi \Psi^{-1}$ в точке $x=0$.

Введем понятие локальной монодромии. Рассмотрим маленькую окружность с центром в нуле и аналитически продолжим вдоль нее $\Psi(x)$ - решение (3). Обозначим результат этого аналитического продолжения $\Psi\left(x e^{2 \pi i}\right)$. Поскольку $A x^{-1}+\mathbf{O}(1)$ - однозначная функция, $\Psi\left(x e^{2 \pi i}\right)$ является решением того же уравнения, а любые два решения одного линейного уравнения отличаются постоянным правым множителем:

$$
\Psi\left(x e^{2 \pi i}\right)=\Psi(x) M .
$$

Эта матрица $M$ и называется локальной монодромией решения $\Psi$ в точке $x=0$.

Домножение $\Psi$ справа на постоянную матрицу приводит к преобразованию подобия $M$ :

$$
\Psi(x) \rightarrow \Psi(x) \Phi_{r} \quad \Rightarrow \quad M \rightarrow \Phi_{r}^{-1} M \Phi_{r},
$$

и сохраняет уравнение; домножение $\Psi$ слева на постоянную матрицу приводит к преобразованию подобия коэффициентов уравнения (3), в частности вычета $A$, - меняет уравнение:

$$
\Psi(x) \rightarrow \Phi_{l} \Psi(x) \quad \Rightarrow \quad A \rightarrow \Phi_{l}^{-1} A \Phi_{l},
$$

и сохраняет монодромию.

Таким образом, имеет смысл рассматривать не “индивидуальное" соответствие $A \leftrightarrow M$, не выдерживающее калибровочных преобразований $\Psi(x) \rightarrow$ $\Phi_{l} \Psi(x) \Phi_{r}$, а соответствие между классами эквивалентности (по сопряжению)

$$
\bigcup_{\Phi \in \operatorname{SL}(2, \mathbb{C})} \Phi^{-1} A \Phi \leftrightarrow \bigcup_{\Phi \in \operatorname{SL}(2, \mathbb{C})} \Phi^{-1} M \Phi .
$$

Такие классы можно характеризовать нормальной жордановой формой содержащихся в них матриц. Мы выберем $\Psi_{\text {sing }}$ так, что

а) вычет $d \Psi_{\text {sing }} \Psi_{\text {sing }}^{-1}$ имеет нормальную жорданову форму:

$$
\frac{d}{d x} \Psi_{\text {sing }}=\left(\frac{A_{\mathrm{J}}}{x}+\mathbf{O}(1)\right) \Psi_{\text {sing }},
$$

$A_{\mathrm{J}}$ - нормальная жорданова форма матрицы $A$ в (3); 
б) локальная монодромия $\Psi_{\text {sing }}(x)$ тоже имеет нормальную жорданову форму:

$$
\Psi_{\text {sing }}\left(x e^{2 \pi i}\right)=\Psi_{\text {sing }}(x) M_{\mathrm{J}},
$$

$M_{\mathrm{J}}$ - нормальная жорданова форма матрицы $M$ в (5).

Пусть $\pm a-$ собственные значения $A$. Если $2 a \notin \mathbb{Z}$, то $A_{\mathrm{J}}$ и $M_{\mathrm{J}}$ диагональны, и, как-то зафиксировав ветвь $x^{a}$, получаем набор

$$
A_{\mathrm{J}}=\left(\begin{array}{cc}
a & 0 \\
0 & -a
\end{array}\right), \quad M_{\mathrm{J}}=\left(\begin{array}{cc}
e^{2 \pi i a} & 0 \\
0 & e^{-2 \pi i a}
\end{array}\right), \quad \Psi_{\mathrm{sing}}=\left(\begin{array}{cc}
x^{a} & 0 \\
0 & x^{-a}
\end{array}\right) .
$$

ЗАмЕчАНИЕ 1. В общем случае, и для полуцелых $a$, локальные исследования (см. [29], [23], [12], [30]) показывают, что $\Psi_{\text {sing }}$ можно выбрать так, что

$$
\Psi_{\text {sing }}=\left(\begin{array}{cc}
x^{a} & 0 \\
0 & x^{-a}
\end{array}\right)\left(\begin{array}{cc}
1 & \tilde{\psi}_{\text {sing }}\left(x \mid M_{\mathrm{J}}\right) \\
0 & 1
\end{array}\right),
$$

где $\tilde{\psi}_{\operatorname{sing}}\left(x \mid M_{\mathrm{J}}\right)$ - функция, определяемая монодромией. Она полиномиально зависит от $\log x$.

1.2. Глобальная теория, изомонодромные деформации. Нашей целью является изучение таких деформаций уравнения, что его монодромии не меняются. Из предыдущего замечания следует, что всегда

$$
\text { "собственное число" } A_{\mathrm{J}}=\frac{1}{2 \pi i} \log \text { “собственное число" } M_{\mathrm{J}} \text {, }
$$

т. е. хорошо известное утверждение:

УтВеРЖДЕНИЕ 1. При непрерывных изомонодромных деформаииях собственные числа вычетов $A^{(k)}$ не меняются - это параметры деформации.

Вернемся к уравнению (1). Рассмотрим его глобально (на $\overline{\mathbb{C}}$ ) по $\lambda$ и локально по параметрам $\lambda_{k}$. Удобна следующая точка зрения и обозначения.

Рассматривается $\mathbb{C P}^{1}$ и набор попарно непересекающихся дисков

$$
\Lambda_{0}, \Lambda_{1}, \ldots, \Lambda_{n} \subset \mathbb{C P}^{1}
$$

Независимой переменной будет положение точки $P \in \mathbb{C P}^{1}$, а параметрами положение точек $P_{k} \in \Lambda_{k}, k=0,1, \ldots, n$.

Мы уже знаем, что в точках $P=P_{k}, k=0,1, \ldots, n$, функция

$$
\Psi=\Psi\left(P ; P_{0}, P_{1}, \ldots, P_{n}\right)
$$

определена не будет (это ее точки ветвления). Обозначим $\Lambda:=\Lambda_{0} \times \cdots \times \Lambda_{n}$ и $\Delta_{\Lambda}$ множество, состоящее из таких $\left(P, P_{0}, \ldots, P_{n}\right)$, что $P$ совпадает с какойнибудь из $P_{k}$. Зададим область определения $\left(\mathbb{C P}^{1} \times \Lambda\right) \backslash \Delta_{\Lambda}$ функции $\Psi$, выбросив из $\mathbb{C P}^{1} \times \Lambda$ множество $\Delta_{\Lambda}$.

ЗАмечание 2 . Матричнозначная функция $\Psi$ как функция $P$ при фиксированных $P_{k}, k=0, \ldots, n$, имеет ветвление в $P_{k}$, так что, на самом деле, для каждого набора $P_{0}, \ldots, P_{n}$ функция $\Psi$ определена не на самой $\mathbb{C P}^{1} \backslash\left\{P_{0}, \ldots, P_{n}\right\}$, 
а на ее универсальной накрывающей. Однако для упрощения речи мы будем пользоваться лексикой многозначных функиий на $\mathbb{C P}^{1}$, подразумевая естественную проекцию на $\mathbb{C P}^{1}$ достаточно малой окрестности обсуждаемой точки.

Введем на $\mathbb{C} \mathbf{P}^{1} \times \Lambda$, как на декартовом произведении многообразий ${ }^{2}$, операторы частного дифберенцирования $d_{\downarrow}$ и $d_{\uparrow}$.

Для любой параметризации $\lambda: \mathbb{C P}^{1} \stackrel{\sim}{\rightarrow} \overline{\mathbb{C}}$ положим $\lambda:=\lambda(P), \lambda_{k}:=\lambda\left(P_{k}\right)$ и для любой функции $f=f\left(\lambda ; \lambda_{0}, \ldots, \lambda_{n}\right)$ определим выражения $d_{\downarrow} f$ и $d_{\uparrow} f$ :

$$
d_{\downarrow} f:=\left.\frac{\partial}{\partial \lambda}\right|_{\lambda_{k}=\text { const }} f d \lambda, \quad d_{\uparrow} f:=\left.\sum_{k=0}^{n} \frac{\partial}{\partial \lambda_{k}}\right|_{\substack{\lambda=\text { const } \\ \lambda_{i}=\text { const } \\ i \neq k}} f d \lambda_{k} .
$$

Они не зависят от параметризации $\lambda$ и корректно определяют операторы "частного дифференцирования", удовлетворяющие соотношениям:

$$
d_{\downarrow}+d_{\uparrow}=d, \quad d_{\downarrow} \wedge d_{\downarrow}=d_{\uparrow} \wedge d_{\uparrow}=0=d \wedge d, \quad d_{\downarrow} \wedge d_{\uparrow}=-d_{\uparrow} \wedge d_{\downarrow} .
$$

Уравнение (1) может быть переписано в форме $d_{\downarrow} \Psi=\omega_{\downarrow} \Psi$, где

$$
\omega_{\downarrow}:=\sum_{k=0}^{n} A^{(k)} d_{\downarrow} \log \left(\lambda(P)-\lambda\left(P_{k}\right)\right)
$$

- мероморфный дифференциал на $\mathbb{C P}^{1}$, все особенности которого - это простые полюсы в $P_{k}$ с вычетами $A^{(k)} \in \operatorname{sl}(2, \mathbb{C})$.

Рассмотрим какое-нибудь решение $\Psi(P)$ этого уравнения (локально, в какой-нибудь области вне дисков $\left.\Lambda_{k}\right)$. Его, как и решение уравнения $(3)$, можно аналитически продолжать вдоль любого пути $\mathscr{L}$, не проходящего через особенности $P_{k}$. Если этот путь окажется замкнутым, мы снова получим два решения одного уравнения (дифференциал $\omega_{\downarrow}$ однозначен); эти решения отличаются постоянным (не зависящим от $P$ ) правым множителем $M_{\mathscr{L}}$ - монодромией, что можно было бы обозначить как $\Psi\left(P e^{2 \pi i \mathscr{L}}\right)=\Psi(P) M_{\mathscr{L}}$.

Нас будут интересовать решения $\Psi$, монодромии которых вдоль всех циклов на $\mathbb{C P}^{1} \backslash \Lambda$ постоянны, не зависят от вариации параметров $P_{k}$ внутри своих $\Lambda_{k}$.

Поскольку решение $\Psi(P)$ определяется уравнением (вычетами $A^{(k)}$ ) с точностью до произвольного правого множителя, поставим вопрос следующим образом:

ЗАДАЧА ИЗОМОНОДРОМНОЙ ДЕФОРМАЦИИ. Как могут непрерывно зависеть вычеты $A^{(k)}$ дифференциала $\omega_{\downarrow}$ от параметров $P_{k}$ (коэффициенты уравнения (1) от параметров $\left.\lambda_{k}\right)$, чтобъ существовала $\Psi\left(P ; P_{0}, \ldots, P_{n}\right)$, монодромии $M_{\mathscr{L}}$ которой вдоль всех ииклов $\mathscr{L}$ на $\mathbb{C P}^{1} \backslash \Lambda$ одни и те же, не меняются при вариации параметров $P_{k} \in \Lambda_{k}$ ?

Любое такое семейство решений $\Psi(P)$ (вместе с соответствующим семейством уравнений), гладко зависящее от параметров $P_{k}$, назовем изомонодромной деформаиией.

\footnotetext{
${ }^{2}$ Потом их, конечно же, ограничим на $\left(\mathbb{C} \mathbf{P}^{1} \times \Lambda\right) \backslash \Delta_{\Lambda}$.
} 
ЗАмечАниЕ 3. Можно доказать (см. [23]), что любая непрерывная изомонодромная деформация может быть параметризована аналитически.

ЗАмечание 4. Существуют еще дискретные преобразования $\Psi$, не меняющие монодромии, но нетривиально меняющие уравнение, это так называемые преобразования Шлезингера. Поскольку целью данной статьи является построение координат на связном гладком многообразии - компоненте изомонодромного подмногообразия, то дискретные преобразования (Шлезингера) намеренно исключены из рассмотрения.

Из (4) следует, что монодромия $\Psi$ при обходе точки $P_{k}$ имеет представление

$$
M^{(k)}=\left(\Psi_{\mathrm{mdr}}^{(k)}\right)^{-1} M_{\mathrm{J}} \Psi_{\mathrm{mdr}}^{(k)} .
$$

Оставим в стороне сложный случай целой разницы собственных значений $a-(-a)=2 a$ матрицы $A_{\mathrm{J}}$, при ненулевых $a$ порождающий дополнительные резонансные компоненты и далеко выходящий за рамки данной статьи (см. [13], [14]). Для него мы просто сформулируем конечный результат (теорема 1 ниже). Сейчас рассмотрим представление (4), где матрица $\Psi_{\text {sing }}$ задана в (6). Если монодромия постоянна, то $\Psi_{\mathrm{mdr}}$ тоже постоянна с точностью до левого диагонального множителя. Однако $\Psi_{\mathrm{mdr}}$ и определена с точностью до такого множителя, поскольку его можно “проносить" через диагональную $\Psi_{\text {sing }}$, относя к $\Psi_{\text {reg. }}$ Так что при изомонодромной деформации (в нерезонансном случае) всегда можно выбрать

$$
\Psi_{\mathrm{mdr}}=\text { const } \in \mathrm{SL}(2, \mathbb{C}) .
$$

Мы получим следующее равенство (10), известное еще Л. Фуксу и лежащее в основе теории шлезингеровых деформаций.

УТВЕРЖДЕНИЕ 2. Если собственные значения вычета матрии, $A^{(k)}$ не полуцелье, то функиия $\Psi:\left(\mathbb{C P}^{1} \times \Lambda\right) \backslash \Delta_{\Lambda} \rightarrow \mathrm{SL}(2, \mathbb{C})$ является решением уравнения (1) и имеет постоянные монодромии тогда и только тогда, когда она регулярна по всем аргументам и в окрестности выколотых точек $P_{k}$ может быть представлена в виде

$$
\Psi=\Psi_{\text {reg }}^{(k)}\left(P ; P_{\bar{i}}\right) \Psi_{\text {sing }}^{(k)}\left(\lambda(P)-\lambda\left(P_{k}\right)\right) \Psi_{\text {mdr }}^{(k)}, \quad P \in \Lambda_{k},
$$

где $\lambda$ - какой-нибудъ голоморфный параметр на $\mathbb{C} \mathbf{P}^{1}, \Psi_{\mathrm{reg}}^{(k)}$ регулярна, $\Psi_{\mathrm{mdr}}^{(k)}$ постоянна, a $\Psi_{\text {sing }}^{(k)}(x)$ - многозначная функиия одной комплексной переменной (6).

ДокАзАтЕЛьство. Необходимость нами доказана, а достаточность очевидна, так как $d_{\downarrow} \Psi \Psi^{-1}$ имеет только простые полюсы и, по построению, все локальные монодромии такой $\Psi$ постоянны.

Для $a=0$ аналогичное утверждение тоже верно, хотя $\Psi_{\operatorname{sing}}$ уже не имеет диагональной формы и доказательство чуть более громоздко, однако для случая $2 a \in \mathbb{N}$ изомонодромная деформация уже не обязана иметь вид (10), хотя 
дебормации (10) все равно остаются изомонодромными. Это мы примем без доказательства, не желая отвлекаться на исследование сложной формы $\Psi_{\text {sing }}$ и ее монодромии в этом случае.

Чтобы выписать уравнение, которому удовлетворяет функция $\Psi$ по параметрам - переменным $\lambda_{k}$, отметим два важных обстоятельства.

Во-первых, в точке $\lambda=\lambda_{k}$ сингулярный множитель не зависит от остальных параметров $\lambda_{i}, i \neq k$, вся зависимость от них сосредоточена в $\Psi_{\mathrm{reg}}$.

Во-вторых, $\Psi_{\operatorname{sing}}$ зависит только от разности $\lambda-\lambda_{k}=x$, так что обе матричнозначные функции - как $\left(\frac{\partial}{\partial \lambda_{k}} \Psi\right) \Psi^{-1}$, так и $\left(\frac{\partial}{\partial \lambda} \Psi\right) \Psi^{-1}$, имеют в $\lambda=\lambda_{k}$ простой полюс с тем же самым вычетом $A^{(k)}$.

Итак, зафиксируем $P$ и все $P_{i}$, кроме одного, кроме $P_{k}$. Рассмотрим

$$
\left.\frac{\partial}{\partial \lambda_{k}}\right|_{\substack{\lambda=\text { const } \\ \lambda=\text { const } \\ i \neq k}} \Psi\left(\lambda ; \lambda_{0}, \ldots, \lambda_{n}\right)=: \frac{\partial}{\partial \lambda_{k}} \Psi(\lambda)
$$

как функцию (одной) комплексной переменной $\lambda$. Она регулярна везде на $\overline{\mathbb{C}}$, кроме, быть может, точек $\lambda_{i}, i=0, \ldots, n$.

ЗАмечаниЕ 5. Особо отметим, что функция $\frac{\partial}{\partial \lambda_{k}} \Psi$ регулярна и в $\infty_{\lambda} \in$ $\mathbb{C P}^{1}$ - той точке, где (произвольно выбранный) параметр $\lambda$ обращается в бесконечность. Эта точка ничем не выделена для $\Psi\left(P ; P_{0}, \ldots, P_{n}\right)$.

Какая сингулярность у $\left(\frac{\partial}{\partial \lambda_{k}} \Psi\right) \Psi^{-1}$ в точках $P_{i}$ ? Рассмотрим представление $(10)$; заметим, что сингулярный член $\Psi_{\operatorname{sing}}^{(i)}\left(\lambda-\lambda_{i}\right) \Psi_{\operatorname{mdr}}^{(i)}$ при $i \neq k$ вообще от $\lambda_{k}$ не зависит, а при $i=k$ зависит от разности $\lambda-\lambda_{k}$. Мы видим, что единственная особенность $\left(\frac{\partial}{\partial \lambda_{k}} \Psi\right) \Psi^{-1}$ как функции $\lambda$ на всей сфере Римана - это простой полюс в $P_{k}$ и с тем же самым вычетом, что и у $\left(\frac{\partial}{\partial \lambda} \Psi\right) \Psi^{-1}$ (см. [31;
уравнение $(14)$, с. 103]):

$$
\frac{\partial}{\partial \lambda_{k}} \Psi=\left(\frac{1}{\lambda_{k}-\lambda} A^{(k)}+R^{(k)}\right) \Psi
$$

где $R^{(k)}=R^{(k)}\left(P_{0}, P_{1}, \ldots, P_{n}\right)$ - какая-то не зависящая от $P$ матрица - значение $\left(\frac{\partial}{\partial \lambda_{k}} \Psi\right) \Psi^{-1}$ в точке $\infty_{\lambda}$.

ТЕорема 1 (Л. Фукс, Л. Шлезингер). Для изомонодромности дебормаици $\Psi$ достаточно выполнения уравнений (11).

В нерезонансном случае, т. е. если отсутствуют натуральные разности собственных значений вычетов $A^{(k)}$, это условие является и необходимым.

ЗАмечАниЕ 6. Несмотря на кажущуюся симметрию (11) и (1), еще раз отметим, что имеется принципиальная разница. Зависимость $d \Psi \Psi^{-1}$ от $\lambda_{k}$ локальна и трансцендентна: $\lambda_{k} \in \lambda\left(\Lambda_{k}\right), A^{(k)}=A^{(k)}\left(\lambda_{0}, \ldots, \lambda_{n}\right)$, в то время как от $\lambda$ обе функции $\left(\frac{\partial}{\partial \lambda_{k}} \Psi\right) \Psi^{-1}$ и $\left(\frac{\partial}{\partial \lambda} \Psi\right) \Psi^{-1}$ зависят рачионально. 
Перепишем уравнения на $\Psi$ в дифференциалах:

$$
\begin{gathered}
d \Psi=\sum_{k=0}^{n}\left(A^{(k)} d \log \left(\lambda-\lambda_{k}\right)+R^{(k)} d \lambda_{k}\right) \Psi \stackrel{\text { def }}{=} \omega \Psi, \quad \omega=\omega_{\downarrow}+\omega_{\uparrow}, \\
\omega_{\downarrow}=\sum_{k=0}^{n} A^{(k)} \frac{d \lambda}{\lambda-\lambda_{k}}, \quad \omega_{\uparrow}=\sum_{k=0}^{n}\left(A^{(k)} \frac{1}{\lambda_{k}-\lambda}+R^{(k)}\right) d \lambda_{k} .
\end{gathered}
$$

Обозначим $R:=\sum_{k=0}^{n} R^{(k)} d \lambda_{k}$. Сразу видно, что

$$
R=d_{\uparrow} \Psi \Psi^{-1}\left(\infty_{\lambda} ; P_{0}, \ldots, P_{n}\right),
$$

поэтому $R$ - плоская связность на $\Lambda_{0} \times \cdots \times \Lambda_{n}=\Lambda$.

С другой стороны, $R$ - любая плоская связность. Действительно, домножение $\Psi$ слева на любую функцию, не зависящую от $P$, монодромии (в частности ее постоянства) не меняет, так что $\Psi\left(\infty_{\lambda} ; P_{0}, \ldots, P_{n}\right)$ можно выбрать любой, и, стало быть, $R$ - любая плоская.

Выпишем уравнения на $A^{(k)}$ и $R^{(k)}$, обеспечивающие разрешимость (12). Их выполнение повлечет существование изомонодромной $\Psi$, принадлежность уравнения изомонодромному слою, т. е. соответствующие $A^{(k)}$ будут являться решением задачи изомонодромной деформации.

Поскольку $\Psi$ - локально однозначная функция, т. е. у любой точки ее области определения (точки $P_{k}$ там не содержатся, они выколоты) есть окрестность, в которой можно выделить однозначную ветвь, то имеют место равенства

$$
\frac{\partial^{2}}{\partial \lambda \partial \lambda_{k}} \Psi=\frac{\partial^{2}}{\partial \lambda_{k} \partial \lambda} \Psi, \quad \frac{\partial^{2}}{\partial \lambda_{i} \partial \lambda_{j}} \Psi=\frac{\partial^{2}}{\partial \lambda_{j} \partial \lambda_{i}} \Psi .
$$

На языке внешних форм эти равенства записываются так: $d^{2} \Psi=0$; подставляя сюда $d \Psi=\omega \Psi$, получаем уравнения $d \omega+\omega \wedge \omega=0$ :

$$
\begin{gathered}
\sum_{k=0}^{n} d A^{(k)} \wedge d \log \left(\lambda-\lambda_{k}\right)+\sum_{i, j=0}^{n}\left(A^{(i)} A^{(j)} d \log \left(\lambda-\lambda_{i}\right) \wedge d \log \left(\lambda-\lambda_{j}\right)\right. \\
\left.+R^{(i)} A^{(j)} d \log \lambda_{i} \wedge d \log \left(\lambda-\lambda_{j}\right)\right)=0, \\
d R+R \wedge R=0 .
\end{gathered}
$$

Приравняв нулю суммарный вычет в каждом полюсе, получим

$$
\begin{gathered}
d A^{(k)}+\left[\sum_{i=0}^{n}\left(A^{(i)} d \log \left(\lambda_{k}-\lambda_{i}\right)+R^{(i)} d \lambda_{i}\right), A^{(k)}\right]=0, \\
\frac{\partial}{\partial \lambda_{k}} R^{(i)}-\frac{\partial}{\partial \lambda_{i}} R^{(k)}+\left[R^{(i)}, R^{(k)}\right]=0 ;
\end{gathered}
$$

здесь использовано обозначение $d \log \left(\lambda_{k}-\lambda_{k}\right) \stackrel{\text { def }}{=} 0$, т. е. слагаемое суммы при $i=k-$ это $R^{(k)} d \lambda_{k}$. Для нерезонансного случая доказана (Л. Шлезингер, [31]), а в резонансном принимается без доказательства следующая теорема. 
ТеОРема 2. Чтобъ члены семейства уравнений (1), непрерывно параметризованного $\lambda_{k}$, имели одинаковые наборы монодромий, достаточно, а в нерезонансном случае необходимо и достаточно, чтобы коэффициенты $A^{(k)}$ удовлетворяли (13) для каких-нибудъ $R^{(k)}$, удовлетворяющих (14).

\section{2. Подмногообразия изомонодромных слоев. Система Шлезингера}

Далее мы будем исследовать только деформации, описываемые уравнениями (13)-(14), назовем их деформациями шлезингеровского типа. В нерезонансном случае все изомонодромные деформации ими и исчерпываются.

Из вида уравнений (13)-(14) следует, что размерность многообразия их решений, т. е. уравнений, имеющих одинаковую монодромию, больше (на три единицы) числа параметров $(n+1)$. Это следует из-за наличия в них $R-$ произвольной плоской $\operatorname{sl}(2, \mathbb{C})$-значной формы на $\Lambda_{0} \times \cdots \times \Lambda_{n}$. Размерность многообразия таких форм именно трu $(3=\operatorname{dim} \operatorname{SL}(2, \mathbb{C}))$, так как $R=d_{\uparrow} \Psi \Psi^{-1}\left(\infty_{\lambda}\right)$, где $\Psi\left(\infty_{\lambda}\right)$ - любая $\mathrm{SL}(2, \mathbb{C})$-значная функция $n+1$ комплексного параметра $P_{k}$.

Уравнения, задающие все многообразие таких изомонодромных деформаций, получаются исключением $R$ из (13)-(14); они, следовательно, не могут иметь вида $d A^{(k)}=F_{k}\left(A^{(0)}, \ldots, A^{(n)}\right)$, где $F_{k}$ - выражение, зависящее от матричных элементов $A^{(i)}, P_{k}$ и $d P_{k}$. Эти уравнения (Гарнье-Пенлеве VI) имеют вид

$$
d G_{k}\left(A^{(0)}, \ldots, A^{(n)}\right)=F_{k}\left(A^{(0)}, \ldots, A^{(n)}\right),
$$

где $G_{k}$ - некоторые функции матричных элементов $A^{(i)}$. По значениям $G_{k}\left(A^{(0)}\right.$, $\left.\ldots, A^{(n)}\right)$ невозможно восстановить сами $A^{(i)}$, только некоторые специфические характеристики набора $A^{(0)}, \ldots, A^{(n)}$ (например, следы всевозможных произведений $\left.A^{(i)} A^{(j)}\right)$.

Уравнения Гарнье-Пенлеве VI будут выведены позже - в разделе 6 , а сейчас выпишем уравнения (Шлезингера) - более сильные - именно вида

$$
d A^{(k)}=F_{k}\left(A^{(0)}, \ldots, A^{(n)}\right), \quad k=0, \ldots, n,
$$

из них будет следовать изомонодромность и выполнение (13). Для получения таких уравнений можно, каким угодно образом, задать матрицы

$$
R^{(k)}=R^{(k)}\left(A^{(0)}, \ldots, A^{(n)}\right),
$$

удовлетворяющие (14). Один из естественных способов - это просто положить $R=0$. Уравнения на $A^{(k)}$, получившиеся в результате такого выбора $R$, называются системой уравнений Шлезингера:

$$
d A^{(k)}+\sum_{\substack{i=0 \\ i \neq k}}^{n}\left[A^{(i)}, A^{(k)}\right] d \log \left(\lambda_{i}-\lambda_{k}\right)=0, \quad k=0, \ldots, n .
$$

Им удовлетворяют деформации шлезингеровского типа, обладающие дополнительным свойством, а именно: те решения этих уравнений, что обладают одинаковыми монодромиями, принимают одно, общее для всех, значение $\Psi\left(\infty_{\lambda}\right)=$ const $\in \operatorname{SL}(2, \mathbb{C})$ в некоторой фиксированной точке, в $\infty_{\lambda} \in \mathbb{C P}^{1}$. 
Положить $R=0$ - не единственно возможный выбор, укажем еще один (кроме дающего систему Шлезингера) способ, который окажется удобным в дальнейшем, при построении канонических координат.

Выберем три различные точки $\infty_{+}, \infty_{-}, \infty_{\star}$ на $\mathbb{C} \mathbf{P}^{1}$. Пусть (пока) они не совпадают ни с одной из $P_{k}$.

Потребуем, чтобы первые строки $\Psi\left(\infty_{+}\right)$и $d_{\downarrow} \Psi\left(\infty_{+}\right)$были линейно зависимы; также пусть линейно зависимы вторые строки $\Psi\left(\infty_{-}\right)$и $d_{\downarrow} \Psi\left(\infty_{-}\right)$.

Такое условие эквивалентно обращению в нуль матричных элементов

$$
\left(d_{\downarrow} \Psi \Psi^{-1}\right)_{12}\left(\infty_{+}\right) \quad \text { и } \quad\left(d_{\downarrow} \Psi \Psi^{-1}\right)_{21}\left(\infty_{-}\right) .
$$

Далее мы убедимся, что в нашем, “одновременно-нетреугольном", случае всегда можно выбрать точки $\infty_{ \pm}$maк, что эти условия выполняются.

Калибровочные преобразования матричнозначной функции $\Psi$ приводят к преобразованию подобия $d_{\downarrow} \Psi \Psi^{-1}(P)$ одной и той же матрицей для всех $P$. Преобразования, не разрушающие ни верхней треугольности (в $\left.P_{+}\right)$, ни нижней треугольности (в $\left.P_{-}\right)$, - это преобразования диагональными матрицами. Зафиксируем диагональный множитель, потребовав постоянства и неравенства нулю (для всех значений параметров $\lambda_{k}$ ) какого-нибудь недиагонального элемента матрицы $\left(\frac{d_{\downarrow}}{d \lambda} \Psi\right) \Psi^{-1}$ в точке $\infty_{\star}$.

Итак, пусть при всех значениях параметров $P_{k}$

$$
\begin{gathered}
\left.\left(d_{\downarrow} \Psi \Psi^{-1}\right)_{12}\right|_{\infty_{+}}=\left.\left(d_{\downarrow} \Psi \Psi^{-1}\right)_{21}\right|_{\infty_{-}}=0, \\
\left.\left(\left(\frac{d_{\downarrow}}{d \lambda} \Psi\right) \Psi^{-1}\right)_{12}\right|_{\infty_{\star}}=1
\end{gathered}
$$

для некоторой параметризации $\lambda$.

Можно показать, что из этих условий определяются, т. е. выражаются через $A^{(k)}$, все матричные элементы $R_{i j}^{(k)}$. Мы этого делать не будем. Приведем только конечную формулу, тот ее вариант, что получится, если поместить $\infty_{+}$, $\infty_{-}, \infty_{\star}$ в точки ветвления $P_{0}, P_{n-1}, P_{n}$ (что и будет сделано ниже). В этом случае вместо треугольности и постоянства недиагонального элемента у $d \Psi \Psi^{-1}$ калибровка однозначно фиксируется наличием этих же свойств у соответствующих вычетов $A^{(k)}$. Уравнения (12) на $\Psi$ принимают вид

$$
\begin{aligned}
d \Psi \Psi^{-1}= & \sum_{k=0}^{n}\left(A^{(k)} d \log \left(\lambda-\lambda_{k}\right)\right. \\
& \left.-\left(\begin{array}{cc}
\frac{A_{11}^{(k)}}{\lambda_{k}-\lambda_{n}}+A_{11}^{(n)} A_{12}^{(k)}\left(\frac{1}{\lambda_{k}-\lambda_{n}}-\frac{1}{\lambda_{k}-\lambda_{0}}\right) & \frac{A_{12}^{(k)}}{\lambda_{k}-\lambda_{0}} \\
\frac{A_{21}^{(k)}}{\lambda_{k}-\lambda_{n-1}} & -(\cdots)
\end{array}\right) d \lambda_{k}\right),
\end{aligned}
$$

здесь опять используем символ $d \log \left(\lambda_{k}-\lambda_{k}\right):=0=: \frac{1}{\lambda_{k}-\lambda_{k}}$. 
3. Гамильтонов формализм и пуассонова динамика на $\operatorname{sl}(2, \mathbb{C})$

Дальнейшее изложение требует введения некоторых понятий теории гамильтоновых систем. Приведем необходимые формулы и дадим пояснения. Утверждения и теоремы ближайших пунктов имеются в учебниках [1], [15], [32].

3.1. Гамильтонов формализм. Пусть $M$ - симплектическое многообразие; на нем задана симплектическая (т.е. внешняя, гладкая, замкнутая, не обращающаяся в нуль) 2-форма:

$$
\omega=\sum_{i, j} \Pi_{i j} d x^{i} \wedge d x^{j}, \quad \Pi_{i j}=-\Pi_{j i} .
$$

Симплектическое многообразие является пуассоновым - скобка Пуассона, кососимметрическое произведение ковекторов (дифференциалов функций)

$$
\{f, g\}=\sum_{i, j} \Pi^{i j} \frac{\partial f}{\partial x^{i}} \frac{\partial g}{\partial x^{j}}, \quad \Pi^{i j}=-\Pi^{j i},
$$

задается обратной матрицей

$$
\left(\Pi_{\ldots}\right)^{-1}=\Pi^{\cdots}: \sum_{k} \Pi_{i k} \Pi^{k j}=\delta_{i}^{j} .
$$

Координаты $x^{1}=p, x^{2}=q$ называются каноническими, если $\omega=d p \wedge d q$, т. e.

$$
\begin{array}{ll}
\Pi \ldots=\left(\begin{array}{cc}
0 & 1 \\
-1 & 0
\end{array}\right), & \Pi_{12}=-\Pi_{21}=1, \\
\Pi^{\cdots}=\left(\begin{array}{cc}
0 & -1 \\
1 & 0
\end{array}\right)=-\Pi \ldots, & \Pi^{12}=-\Pi^{21}=-1,
\end{array}
$$

и, значит, для канонических координат

$$
\left\{x^{1}, x^{2}\right\}=\Pi^{12}=\{p, q\}=-1 .
$$

Система уравнений Гамильтона - (гамильтоново) векторное поле на $M-$ в канонических координатах имеет вид

$$
\left\{\begin{array}{l}
\dot{q}=\frac{\partial}{\partial p} H \\
\dot{p}=-\frac{\partial}{\partial q} H
\end{array}\right.
$$

где $H$ - функция на $M$ (гамильтониан). Система (18) может быть записана по-разному:

а) симплектическая формулировка:

как условие того, что направление $d p: d q: d t$ в расширенном фазовом пространстве $M^{\Lambda}:=M \times \Lambda \stackrel{\pi_{M}}{\longrightarrow} M$ является направлением вырождения 2-формы $\Omega$ :

$$
\Omega=\pi_{M}^{*} \omega-d H \wedge d t=d p \wedge d q-d H \wedge d t
$$


б) пуассонова формулировка:

векторное поле можно задать, указав, как по его направлению будут дифференцироваться всевозможные скалярные поля (функции).

Гамильтоново векторное поле с гамильтонианом $H$ дифференцирует по своему направлению произвольную функцию $f$ следующим образом:

$$
\dot{f}=-\{H, f\},
$$

именно при таком выборе знака для координатных функций $x^{1}=p, x^{2}=q$ будем иметь каноническую систему (18):

$$
\begin{aligned}
& \dot{q}=\dot{x}^{2}=-\left\{H, x^{2}\right\}=-\Pi^{i j} \frac{\partial H}{\partial x^{i}} \frac{\partial x^{2}}{\partial x^{j}}=-\Pi^{12} \frac{\partial H}{\partial x^{1}}=\frac{\partial}{\partial p} H, \\
& \dot{p}=\dot{x}^{1}=-\left\{H, x^{1}\right\}=-\Pi^{i j} \frac{\partial H}{\partial x^{i}} \frac{\partial x^{1}}{\partial x^{j}}=-\Pi^{21} \frac{\partial H}{\partial x^{2}}=-\frac{\partial}{\partial q} H .
\end{aligned}
$$

Немного усложним ситуацию. Пусть теперь матрица $\Pi^{i j}$ необратима, $M$ - пуассоново, но не симплектическое многообразие; тогда форма $\omega$ из (19) не может быть задана с помощью элементов обратной матрицы $\Pi_{i j}$ (она не существует), но (20) тем не менее имеет смысл и задает гамильтоново векторное поле.

В этом случае имеется набор нетривиальных функций (Казимира), скобка Пуассона с которыми равна нулю всегда (следствие необратимости $\Pi^{i j}$ ). Интегральные линии поля (20) в этом случае лежат на поверхностях уровня функций Казимира.

Опять изменим ситуацию. Пусть снова $M$ симплектическое (и пуассоново), но теперь имеется не одно время " $t$ ", а несколько "времен" - переменных $\lambda_{0}, \lambda_{1}, \ldots, \lambda_{n}$, являющихся координатными функциями на многообразии $\Lambda$. Пусть зависимость от них задается гамильтонианами $H_{0}, H_{1}, \ldots, H_{n}$, зависящими как от времен $\lambda_{i}$, так и от координат на $M$. Нам требуются новые понятия; введем их, следуя [15], [1].

Слоением ( $k$-мерным) какого-либо $N$-мерного многообразия $M^{\Lambda}$ называется такая совокупность $k$-мерных поверхностей (слоев слоения) $\mathscr{F} \chi \subset M^{\Lambda}$, что через каждую точку многообразия $M^{\Lambda}$ проходит одна и только одна гладкая $k$-мерная поверхность $\mathscr{F} \chi$, гладко зависящая от точки многообразия:

$$
M^{\Lambda}=\coprod_{\chi} \mathscr{F} \chi
$$

значок $\chi$ нумерует слои.

Распределением ( $k$-мерным) называется гладкое поле $k$-мерных касательных направлений, т. е. функция, ставящая в соответствие каждой точке $x \in M^{\Lambda}$ $k$-мерное подпространство $\mathscr{L}(x)$ касательного пространства $T_{x} M^{\Lambda} \supset \mathscr{L}(x)$.

Распределение называется интегрируемым, если имеется слоение, касательные $\mathscr{L}$ к слоям $\mathscr{F} \chi$ которого $\left(\mathscr{L}\right.$ - линейные подмногообразия $T M^{\Lambda}$ размерности $\operatorname{dim} \mathscr{L}=\operatorname{dim} \mathscr{F}_{\chi}=k$ ) образуют это распределение.

ОБОЗНАчЕНИЕ 2. Обозначать распределения будем строчной буквой в квадратных скобках $-[h]$. 
В предыдущем, одномерном, случае слои $\mathscr{F} \chi-$ это интегральные линии гамильтонова поля - решения

$$
\bigcup_{t}(p(t), q(t), t) \subset M^{\Lambda}
$$

(одномерные) подпространства $\mathscr{L}(x)$ - это прямые, задаваемые уравнениями Гамильтона, т. е.

$$
(d p: d q: d t)=(-\{H, p\},-\{H, q\}, 1) .
$$

Одномерное распределение всегда интегрируемо.

Пусть $M^{\Lambda}=M \times \Lambda$ - расширенное фазовое пространство гамильтоновой системы с фазовым пространством $M$ и $(n+1)$-мерным $\Lambda \ni\left(\lambda_{0}, \ldots, \lambda_{n}\right)-$ пространством "времен”.

Распределение $[h]$ в $M^{\Lambda}$ может быть задано 1-формой $h \in T^{*} M^{\Lambda}$ вида

$$
h=\sum_{j=0}^{n} H_{j} d \lambda_{j}
$$

Пусть $M$ - симплектическое многообразие с формой $\omega \in \bigwedge^{2} T^{*} M$. Плоскости $((n+1)$-мерные) распределения $[h]$ могут быть заданы аналогично формуле (19) - как подпространства, на которых форма $\Omega$,

$$
\Omega=\pi_{M}^{*} \omega-d h=\pi_{M}^{*} \omega-\sum_{j=0}^{n} d H_{j} \wedge d \lambda_{j},
$$

вырождена, т. е.

$$
\vec{\xi}_{h} \in[h] \quad \Leftrightarrow \quad \Omega\left(\vec{\xi}, \vec{\xi}_{h}\right)=0 \forall \vec{\xi} \in T M^{\Lambda}
$$

Распределения, заданные гамильтонианами $H_{k}, k=0,1, \ldots, n, n>0$, интегрируемы (задают слоение) тогда и только тогда, когда потоки, задаваемые разными $H_{k}$, коммутируют. Это происходит, когда $H_{k}$ удовлетворяют соотношениям (уравнениям нулевой кривизны):

$$
\left\{\frac{\partial}{\partial \lambda_{j}} H_{i}-\frac{\partial}{\partial \lambda_{i}} H_{j}+\left\{H_{i}, H_{j}\right\}, f\right\}=0 \quad \forall f,
$$

т. е. $\left(H_{i}\right)_{\lambda_{j}}-\left(H_{j}\right)_{\lambda_{i}}+\left\{H_{i}, H_{j}\right\}-$ функция Казимира, например, нуль.

3.2. Скобка Ли-Пуассона. Важнейшим примером пространств, обладающих пуассоновой структурой, являются пространства, двойственные к какойнибудь алгебре Ли. Сначала скобка определяется на линейных функциях (на этих двойственных пространствах), а уж потом, по линейности и правилу Лейбница, распространяется и на всевозможные функции.

Продемонстрируем, как эта схема действует в случае алгебры Ли $\operatorname{sl}(2, \mathbb{C})$. Мы апеллируем к геометрической интуиции и изоморфизму алгебр Ли $\mathrm{su}(2) \simeq$ $\left(\mathbb{R}^{3},[\cdot, \cdot]\right)$, позволяющему рассматривать $\operatorname{sl}(2, \mathbb{C}) \supset \mathrm{su}(2)$ как комплексификацию алгебры Ли вещественных трехмерных векторов с коммутатором - векторным произведением $[\cdot, \cdot]$. Будем называть элементы $\mathrm{sl}(2, \mathbb{C})$ векторами, а элементы $\mathrm{sl}^{*}(2, \mathbb{C})$, т. е. линейные функции на $\mathrm{sl}(2, \mathbb{C}),-$ ковекторами. 
Кроме коммутатора - "векторного произведения", на $\operatorname{sl}(2, \mathbb{C})$ определено и скалярное произведение (Киллинга):

$$
\langle A, B\rangle=\operatorname{Tr} A B
$$

Пользуясь тем, что оно невырождено - только нулевой вектор ортогонален всему пространству:

$$
\langle\sigma, A\rangle=0 \forall A \Rightarrow \sigma=0,
$$

можно отождествить $\operatorname{sl}(2, \mathbb{C})$ и $\mathrm{sl}^{*}(2, \mathbb{C})$. Линейный изоморфизм между ними строится так: вектору $A \in \operatorname{sl}(2, \mathbb{C})$ ставится в соответствие ковектор $|A\rangle \in$ $\mathrm{sl}^{*}(2, \mathbb{C})$ скалярного умножения на этот $A$ :

$|A\rangle$ - линейное отображение $\operatorname{sl}(2, \mathbb{C}) \rightarrow \mathbb{C}$, которое каждому $B \in \operatorname{sl}(2, \mathbb{C})$ ставит в соответствие $\langle B, A\rangle \in \mathbb{C}$; изоморфность следует из невырожденности $\langle\cdot, \cdot\rangle$.

Элементы второго сопряженного пространства $\mathrm{sl}^{* *}(2, \mathbb{C})$ - это линейные функции на $\mathrm{sl}^{*}(2, \mathbb{C})$ - элементы самого $\mathrm{sl}(2, \mathbb{C}) \simeq \mathrm{sl}^{* *}(2, \mathbb{C})$ :

$A \in \mathrm{sl}(2, \mathbb{C})$, как элемент $\mathrm{sl}^{* *}(2, \mathbb{C})$, является функцией на $\mathrm{sl}^{*}(2, \mathbb{C})$, которая каждому $\widetilde{A} \in \mathrm{sl}^{*}(2, \mathbb{C})-$ функционалу $\operatorname{sl}(2, \mathbb{C}) \rightarrow \mathbb{C}-$ ставит в соответствие число - значение этого функционала на $A$ :

$$
A \in \mathrm{sl}^{* *}(2, \mathbb{C}): \mathrm{sl}^{*}(2, \mathbb{C}) \ni \widetilde{A} \mapsto \widetilde{A}(A) \in \mathbb{C} .
$$

Типичный пример линейной функции на $\mathrm{sl}(2, \mathbb{C})$ (элемента $\left.\mathrm{sl}^{*}(2, \mathbb{C})\right)$ - это какой-нибудь матричный элемент $A_{i j}$; соответственно линейная функция на $\mathrm{sl}^{*}(2, \mathbb{C})$ - это матричный элемент $A_{i j}$ ковектора $|A\rangle$. Какие элементы $\mathrm{sl}(2, \mathbb{C})$ соответствуют $A_{11}, A_{12}, A_{21}$ при отождествлении $\mathrm{sl}(2, \mathbb{C}) \simeq \mathrm{sl}^{* *}(2, \mathbb{C})$ ?

Обозначим

$$
\sigma_{3}:=\left(\begin{array}{cc}
1 & 0 \\
0 & -1
\end{array}\right), \quad \sigma_{-}:=\left(\begin{array}{cc}
0 & 0 \\
1 & 0
\end{array}\right), \quad \sigma_{+}:=\left(\begin{array}{cc}
0 & 1 \\
0 & 0
\end{array}\right)
$$

очевидно, что ${ }^{3}$

$$
A_{11}=\left\langle A, \frac{\sigma_{3}}{2}\right\rangle, \quad A_{12}=\left\langle A, \sigma_{-}\right\rangle, \quad A_{21}=\left\langle A, \sigma_{+}\right\rangle .
$$

Функция на sl* - это элемент sl - на нем вычисляются значения функционалов из $\mathrm{sl}^{*}$. По определению, скобка Ли-Пуассона между (линейными) функциями $f_{1}, f_{2} \in\left(\mathrm{sl}^{*}(2, \mathbb{C})\right)^{*}$ на $\mathrm{sl}^{*}(2, \mathbb{C})$ полагается равной их коммутатору:

$$
\left\{f_{1}, f_{2}\right\}_{\mathrm{LP}}=f_{3} \quad \Leftrightarrow \quad F_{3}=\left[F_{1}, F_{2}\right],
$$

если

$$
\mathrm{sl}^{* *}(2, \mathbb{C}) \ni f_{i} \leftrightarrow F_{i} \in \operatorname{sl}(2, \mathbb{C})
$$

\footnotetext{
3Этим объясняется выбор в этой статье базиса $\sigma_{3}, \sigma_{ \pm}$в качестве стандартного - эти векторы сопряжены матричным элементам.
} 
Таким образом, $\left\{A_{11}, A_{12}\right\}_{\mathrm{LP}}$ - это матричный элемент ковектора, соответствующий коммутатору $\frac{\sigma_{3}}{2}$ и $\left(\begin{array}{ll}0 & 0 \\ 1 & 0\end{array}\right):\left[\frac{\sigma_{3}}{2},\left(\begin{array}{ll}0 & 0 \\ 1 & 0\end{array}\right)\right]=-\left(\begin{array}{ll}0 & 0 \\ 1 & 0\end{array}\right)$. Поскольку $\left(\begin{array}{ll}0 & 0 \\ 1 & 0\end{array}\right) \leftrightarrow A_{12}$, то

$$
\left\{A_{11}, A_{12}\right\}_{\mathrm{LP}}=-A_{12}
$$

Аналогично,

$$
\left\{A_{11}, A_{21}\right\}_{\mathrm{LP}}=A_{21}, \quad\left\{A_{21}, A_{12}\right\}_{\mathrm{LP}}=2 A_{11} .
$$

Одной формулой все эти равенства записываются так:

$$
\left\{A_{i j}, A_{k l}\right\}_{\mathrm{LP}}=-A_{i l} \delta_{j k}+A_{k j} \delta_{l i} .
$$

ЗАмЕчАНИЕ 7. Из общей теории следует (см. [32], [33]), что симплектическим пространством является орбита $\mathscr{O}$ какого-нибудь элемента сопряженного этой алгебре Ли пространства; орбита, заметаемая этим элементом при коприсоединенном действии на него самой группы.

Скобка Пуассона $\{\cdot, \cdot\}_{\mathscr{O}}$, канонически соответствующая этой симплектической структуре, согласована со скобкой Ли-Пуассона объемлющего пространства (сопряженного алгебре Ли) следующим образом.

Пусть $f$ и $g$ - функции на сопряженном алгебре (объемлющем) пространстве, a $f_{\mathscr{O}}$ и $g_{\mathscr{O}}-$ их сужения на орбиту $\mathscr{O}$. Тогда значение $\{f, g\}_{\mathrm{LP}}$ в точках орбиты, т. е. сужение $\left.\{f, g\}_{\mathrm{LP}}\right|_{\mathscr{O}}$, совпадает со значением скобки $\{\cdot, \cdot\}_{\mathscr{O}}$, вычисленной на $f_{\mathscr{O}}$ и $g_{\mathscr{O}}$ :

$$
\left\{f_{\mathscr{O}}, g_{\mathscr{O}}\right\}_{\mathscr{O}}=\left.\{f, g\}_{\mathrm{LP}}\right|_{\mathscr{O}} .
$$

Не будем далее писать индексы "LP" и " $\mathscr{O}$ " у скобок, подразумевая сужение функций на соответствующие подмногообразия (орбиты), где значения этих скобок совпадают.

В нашем случае $\operatorname{sl}(2, \mathbb{C})$ легко видеть, что

$$
\left\{A_{11}, \log A_{12}\right\}=\left\{A_{22}, \log A_{21}\right\}=-1,
$$

следовательно, и на орбите $\mathscr{O}$ канонически-сопряженными будут пары переменных $\left(A_{11}, \log A_{12}\right)$, а также $\left(A_{22}=-A_{11}, \log A_{21}\right)$.

Для овеществленного случая $\mathrm{su}(2) \subset \mathrm{sl}(2, \mathbb{C}), \mathrm{SU}(2) \subset \mathrm{SL}(2, \mathbb{C})$ эти утверждения имеют прозрачную геометрическую иллюстрацию.

Относительно введенного на $\operatorname{su}(2) \subset \operatorname{sl}(2, \mathbb{C})$ скалярного произведения $\langle\cdot, \cdot\rangle$ ортонормированным является базис $i \sigma_{k} / 2$, где $\sigma_{k}, k=1,2,3,-$ матрицы Паули.

Возьмем какой-нибудь элемент сопряженного пространства квадрата длины $R^{2}$, например $\left|R i \sigma_{3} / 2\right\rangle$, и подействуем на него всевозможными элементами $\mathrm{SU}(2) \sim \mathrm{SO}(3)$, т. е. вращениями. Получится сфера, которую, как известно, можно параметризовать двумя углами - широтой $\theta \in[-\pi / 2, \pi / 2]$ и долготой $\varphi \in[0,2 \pi[$ :

$$
A=i \frac{R}{2}\left(\begin{array}{cc}
\sin \theta & e^{i \varphi} \cos \theta \\
e^{-i \varphi} \cos \theta & -\sin \theta
\end{array}\right) \in \mathrm{su}(2), \quad|A\rangle \in \mathrm{su}^{*}(2) .
$$


Как мы видели, согласно общей теории, канонические координаты - это $p=A_{11}$ и $q=\log A_{12}$ :

$$
d p \wedge d q=i \frac{R}{2} \cos \theta d \theta \wedge(d \log \cos \theta+i d \varphi)=\frac{R}{2} \cos \theta d \varphi \wedge d \theta,
$$

т. е. знакомый элемент площади сферы (с постоянным множителем $R / 2$ ).

ЗАмечАниЕ 8. Здесь уместно было бы сослаться на Архимеда. По-видимому, он был первым, кто открыл совпадение (форм) площади сферы и описанного вокруг нее цилиндра.

Действительно, пусть координата вдоль образующей есть $z=R \sin \theta$, угловая координата на направляющей окружности есть $\varphi$; Архимед нашел, что

$$
d \varphi \wedge d z=R \cos \theta d \varphi \wedge d \theta .
$$

На орбите

$$
d A_{11}=\left(i \frac{R}{2}\right) d \sin \theta, \quad d \log A_{12}=i d \varphi+d \text { "функция } \theta \text { ", }
$$

значит,

$$
2 d A_{11} \wedge d \log A_{12}=d \varphi \wedge d z=R \cos \theta d \varphi \wedge d \theta .
$$

Таким образом, в привычном нам случае $\mathbb{R}^{3}$ иллюстрируемое общее утверждение о симплектичности орбиты коприсоединенного представления алгебры Ли является утверждением, что сфера с 2-формой площади, наследованной из оббемлющего $\mathbb{R}^{3}$, является симплектическим пространством.

3.3. Пуассонова структура системы Шлезингера. Рассмотрим теперь $n+1$ экземпляр пространства $\mathrm{sl}^{*}(2, \mathbb{C}) \simeq \operatorname{sl}(2, \mathbb{C})$ и их декартово произведение $\operatorname{sl}(2, \mathbb{C})^{n+1}$. Очевидно, что это пуассоново пространство со скобкой

$$
\left\{A_{i j}^{\left(m_{1}\right)}, A_{k l}^{\left(m_{2}\right)}\right\}=\delta_{m_{1} m_{2}}\left(\delta_{i l} A_{k j}^{\left(m_{1}\right)}-\delta_{j k} A_{i l}^{\left(m_{1}\right)}\right)
$$

- нулевой между различными декартовыми сомножителями и скобкой Ли-Пуассона внутри одного экземпляра $\operatorname{sl}(2, \mathbb{C})$.

Рассмотрим теперь $a_{i k}:=\left\langle A^{(i)}, A^{(k)}\right\rangle$ - это функция на $\operatorname{sl}(2, \mathbb{C})^{n+1} ;$ вычислим скобку между матричными элементами $A_{s t}^{(r)}$ какой-нибудь $A^{(r)} \in \operatorname{sl}(2, \mathbb{C})$ и $a_{i k}$.

Во-первых, очевидно, что если $i \neq r \neq k$, то $\left\{a_{i k}, A_{s t}^{(r)}\right\}=0$. Если $i=k$, то $a_{k k}=$ const и $\left\{a_{k k}, A_{s t}^{(r)}\right\}=0$, так что единственный нетривиальный случай это

$$
\begin{aligned}
\left\{a_{i r}, A_{s t}^{(r)}\right\} & =\sum_{m_{1}, m_{2}}\left\{A_{m_{1} m_{2}}^{(i)} A_{m_{2} m_{1}}^{(r)}, A_{s t}^{(r)}\right\} \\
& =\sum_{m_{1}, m_{2}} A_{m_{1} m_{2}}^{(i)}\left(\delta_{m_{2} t} A_{s m_{1}}^{(r)}-\delta_{s m_{1}} A_{m_{2} t}^{(r)}\right) \\
& =\sum_{m_{1}, m_{2}}\left(A_{s m_{1}}^{(r)} A_{m_{1} t}^{(i)}-A_{s m_{2}}^{(i)} A_{m_{2} t}^{(r)}\right)=\left(\left[A^{(r)}, A^{(i)}\right]\right)_{s t} .
\end{aligned}
$$


Впоследствии нам потребуются также скобки

$$
\left\{a_{i j}, a_{k l}\right\}=\left\{\left\langle A^{(i)}, A^{(j)}\right\rangle,\left\langle A^{(k)}, A^{(l)}\right\rangle\right\},
$$

вычислим заодно и их.

Если среди индексов $i, j, k, l$ нет совпадающих, то $\left\{a_{i j}, a_{k l}\right\}=0$, так как отличны от нуля только скобки Пуассона матричных элементов из одного декартова сомножителя $\operatorname{sl}(2, \mathbb{C})$.

Кроме того, легко проверить, что $\left\{a_{i j}, a_{k k}\right\}=0$ для любых $i, j, k$, потому что $a_{k k} / 2$ это квадрат собственного значения $A^{(k)}$ - функция Казимира скобки Ли-Пуассона, что следует из формулы (25):

$$
\left\{A_{i j}, A_{12} A_{21}+A_{11}^{2}\right\}=0 \quad \forall i, j \in\{1,2\} .
$$

Из косой симметрии $\{\cdot, \cdot\}$ вытекает, что $\left\{a_{i j}, a_{i j}\right\}=0$ для любых $i, j$; остается случай

$$
\left\{a_{i k}, a_{j k}\right\}, \quad i \neq j \neq k \neq i \text {. }
$$

В силу равенства (28)

$$
\left\{A_{e m}^{(k)},\left\langle A^{(k)}, A^{(j)}\right\rangle\right\}=-\left(\left[A^{(k)}, A^{(j)}\right]\right)_{e m},
$$

поэтому если $i$ не совпадает ни с $j$, ни с $k$, то

$$
\begin{aligned}
\left\{a_{i k}, a_{k j}\right\} & =\sum_{e, m} A_{e m}^{(i)}\left\{A_{e m}^{(k)},\left\langle A^{(k)}, A^{(j)}\right\rangle\right\} \\
& =-\left\langle A^{(i)},\left[A^{(k)}, A^{(j)}\right]\right\rangle .
\end{aligned}
$$

Теперь, благодаря косой симметрии смешанного произведения $\langle[A, B], C\rangle$, можно снять ограничения на несовпадение индексов - в обеих частях будет нуль, так что всегда

$$
\left\{a_{i k}, a_{k j}\right\}=\left\langle\left[A^{(i)}, A^{(j)}\right], A^{(k)}\right\rangle=: f_{i j k},
$$

т. е. скобки от попарных скалярных произведений трех векторов антисимметричны относительно перестановки пары из этих векторов и не меняются при циклической перестановке.

Обозначим теперь

$$
h:=\sum_{i<j} a_{i j} d \log \left(\lambda_{i}-\lambda_{j}\right)=: \sum_{k=0}^{n} H_{k} d \lambda_{k},
$$

т. e.

$$
H_{k}:=\sum_{i \neq k} \frac{a_{i k}}{\lambda_{k}-\lambda_{i}} .
$$

Уравнения Гамильтона, соответствующие гамильтониану $H_{k}$ в пространстве, снабженном скобкой Ли-Пуассона (27), будут такие (по индексам " $r$ " и " $k$ " в этих формулах суммирования нет!):

$$
\frac{d A_{s t}^{(r)}}{d \lambda_{k}}=-\left\{H_{k}, A_{s t}^{(r)}\right\}=\sum_{i \neq k} \frac{-1}{\lambda_{k}-\lambda_{i}}\left\{a_{i k}, A_{s t}^{(r)}\right\} .
$$


Если $k \neq r$, то

$$
\frac{d A_{s t}^{(r)}}{d \lambda_{k}}=\sum_{i \neq k} \frac{-1}{\lambda_{k}-\lambda_{i}}\left\{a_{i k}, A_{s t}^{(r)}\right\}=\frac{-1}{\lambda_{k}-\lambda_{r}}\left\{a_{k r}, A_{s t}^{(r)}\right\}=\frac{\left(\left[A^{(k)}, A^{(r)}\right]\right)_{s t}}{\lambda_{k}-\lambda_{r}},
$$

если же $k=r$, то

$$
\frac{d A_{s t}^{(r)}}{d \lambda_{r}}=\sum_{i \neq r} \frac{1}{\lambda_{r}-\lambda_{i}}\left\{a_{i r}, A_{s t}^{(r)}\right\}=-\sum_{i \neq r} \frac{\left(\left[A^{(r)}, A^{(i)}\right]\right)_{s t}}{\lambda_{r}-\lambda_{i}},
$$

это совпадает с (15) - системой уравнений Шлезингера.

Мы доказали, что для любого $k \in 0,1, \ldots, n$ линия, являющаяся решением уравнений Шлезингера, совпадает с траекторией гамильтоновой системы с гамильтонианом

$$
H_{k}=h\left(\partial_{\lambda_{k}}\right)=\sum_{i \neq k} \frac{a_{i k}}{\lambda_{k}-\lambda_{i}} .
$$

Вычислим $\left\{H_{i}, H_{j}\right\}$ :

$$
\begin{aligned}
&\left\{H_{i}, H_{j}\right\}= \sum_{m_{1}, m_{2}} \frac{1}{\lambda_{i}-\lambda_{m_{1}}} \frac{1}{\lambda_{j}-\lambda_{m_{2}}}\left\{a_{i m_{1}}, a_{j m_{2}}\right\} \\
&= \sum_{m}\left(\frac{1}{\lambda_{i}-\lambda_{m}} \frac{1}{\lambda_{j}-\lambda_{m}}\left\{a_{i m}, a_{m j}\right\}+\frac{1}{\lambda_{i}-\lambda_{j}} \frac{1}{\lambda_{j}-\lambda_{m}}\left\{a_{i j}, a_{j m}\right\}\right. \\
&\left.\quad+\frac{1}{\lambda_{i}-\lambda_{m}} \frac{1}{\lambda_{j}-\lambda_{i}}\left\{a_{i m}, a_{j i}\right\}\right) \\
&= \sum_{m}\left(\frac{f_{i j m}}{\left(\lambda_{m}-\lambda_{i}\right)\left(\lambda_{m}-\lambda_{j}\right)}\right. \\
&\left.=\sum_{m} \frac{f_{i m j}}{\left(\lambda_{i}-\lambda_{j}\right)\left(\lambda_{j}-\lambda_{m}\right)}+\frac{\left.f_{m j i}-\lambda_{i j m}\right)\left(\lambda_{j}-\lambda_{m}\right)}{\left(\lambda_{j}-\lambda_{i}\right)\left(\lambda_{i}-\lambda_{m}\right)}\right)
\end{aligned}
$$

Поскольку

$$
\frac{\partial}{\partial \lambda_{i}} H_{j}=\frac{\partial}{\partial \lambda_{j}} H_{i}=\frac{\partial^{2}}{\partial \lambda_{i} \partial \lambda_{j}} a_{i j} \log \left(\lambda_{i}-\lambda_{j}\right),
$$

условия интегрируемости (22) выполнены. Распределение, задаваемое формой

$$
h=\sum_{i<j}\left\langle A^{(i)}, A^{(j)}\right\rangle d \log \left(\lambda_{i}-\lambda_{j}\right),
$$

является интегрируемым и совпадает с распределением, задаваемым системой уравнений Шлезингера.

УТВЕРЖДЕНИЕ 3. На некотором подмногообразии пуассонова (скобка (27)) пространства $\mathrm{sl}(2, \mathbb{C})^{n+1}$ система Шлезингера (15) задает слоение, определяемое там формой

$$
h=\sum_{i<j} a_{i j} d \log \left(\lambda_{i}-\lambda_{j}\right)
$$




\section{4. Построение многообразия $M_{a_{\bar{i}}}-$ фазового пространства гамильтоновой системы Гарнье-Пенлеве VI}

4.1. Орбиты коприсоединенного представления $\operatorname{SL}(2, \mathbb{C})$. Результаты этого пункта - элементарные следствия явной формулы $\omega=d A_{11} \wedge d \log A_{12}$ для симплектической формы на орбите $\mathrm{SL}(2, \mathbb{C})$ (см. замечание 8 на с. 74$)$.

Рассмотрим произвольный ненулевой ковектор $\langle A| \in \mathrm{sl}^{*}(2, \mathbb{C})$ и симплектическое пространство $\mathscr{O}$,

$$
\mathscr{O}:=\bigcup_{g \in \operatorname{SL}(2, \mathbb{C})}\left\langle g^{-1} A g\right| \subset \mathrm{sl}^{*}(2, \mathbb{C}),
$$

заметаемое этим ковектором при действии на него $\mathrm{SL}(2, \mathbb{C})$.

Если $A_{1}$ и $A_{2}$ - какие-нибудь ненулевые векторы из $\mathrm{sl}(2, \mathbb{C})$, то их можно совместить элементом $g \in \mathrm{SL}(2, \mathbb{C})$ тогда и только тогда, когда совпадают их "квадраты длин" - произведения на себя:

$$
\forall A_{i} \neq 0 \quad \exists g \in \mathrm{SL}(2, \mathbb{C}): g^{-1} A_{1} g=A_{2} \quad \Leftrightarrow \quad\left\langle A_{1}, A_{1}\right\rangle=\left\langle A_{2}, A_{2}\right\rangle,
$$

следовательно, пространство $\mathscr{O}$ характеризуется единственным параметром комплексным числом $R^{2}:=\langle A, A\rangle \in \mathbb{C}$, так это пространство и будем обозначать $-\mathscr{O}_{R^{2}}$.

Пусть сначала $\langle A, A\rangle=R^{2} \neq 0$, тогда $\mathscr{O}_{R^{2}}$ представляет собой множество всевозможных комплексных матриц с фиксированным определителем $-R^{2} / 2$. Такие матрицы находятся во взаимнооднозначном соответствии с точками аффинной квадрики - гладкой алгебраической поверхности

$$
X_{1} X_{2}+X_{3}^{2}=\frac{R^{2}}{2} \neq 0 \quad \leftrightarrow \quad\left(\begin{array}{cc}
X_{3} & X_{1} \\
X_{2} & -X_{3}
\end{array}\right) \in \mathscr{O}_{R^{2}} .
$$

Симплектическая форма в координатах $X_{i}$ задается равенствами

$$
\begin{aligned}
\omega_{R^{2}} & =d X_{3} \wedge d \log X_{1}=-d X_{3} \wedge d \log X_{2} \\
& =d \frac{\sqrt{R^{2} / 2}+X_{3}}{X_{1}} \wedge d X_{1}=d \frac{\sqrt{R^{2} / 2}-X_{3}}{X_{2}} \wedge d X_{2} \\
& =d \frac{X_{2}}{\sqrt{R^{2} / 2}-X_{3}} \wedge d X_{1}=d \frac{X_{1}}{\sqrt{R^{2} / 2}+X_{3}} \wedge d X_{2} .
\end{aligned}
$$

Эти равенства имеют место, поскольку

$$
X_{1} X_{2}=\left(\sqrt{\frac{R^{2}}{2}}-X_{3}\right)\left(\sqrt{\frac{R^{2}}{2}}+X_{3}\right) .
$$

Кроме того, так как $R^{2} \neq 0$, то всегда хотя бы одно из чисел $\sqrt{R^{2} / 2}+X_{3}$ или $\sqrt{R^{2} / 2}-X_{3}$ отлично от нуля, и соответствующее равенство задает там форму $\omega_{R^{2}}$.

Мы видели, что в вещественном случае форма $\omega_{R^{2}}$ с точностью до множителя $R / 2$ совпадает с формой площади, индуцированной на квадрике

$$
X^{2}+Y^{2}+Z^{2}=R^{2}, \quad X_{1}=x+i y, \quad X_{2}=x-i y, \quad X_{3}=z
$$

объемлющим пространством $\mathbb{R}^{3} \ni(x, y, z)$. 
Рассмотрим теперь случай $R^{2}=0$. Все вышесказанное относится и к этому случаю, за тем исключением, что нулевая матрица $X_{1}=X_{2}=X_{3}=0$ не принадлежит орбите ненулевого элемента $\langle A| \neq 0, \operatorname{det} A=0$, так что из конуса $X_{1} X_{2}+X_{3}^{2}=0$ нужно выкинуть одну точку - вершину $X_{1}=X_{2}=X_{3}=0$. Симплектическое пространство $\mathscr{O}_{0}$ представляет собой гладкую часть квадрики $X_{1} X_{2}+X_{3}^{2}=0$.

ЗАмечАниЕ 9. Форма $\omega_{0}$ в вещественном случае представляет собой форму площади не конуса, а цилиндра.

Действительно, снова введем вещественные $(x, y, z)$ :

$$
x^{2}+y^{2}=z^{2}, \quad X_{1}=x+i y, \quad X_{2}=x-i y, \quad X_{3}=i z,
$$

и цилиндрические координаты $(\rho, \varphi, z)$ :

$$
x=\rho \cos \varphi, \quad y=\rho \sin \varphi, \quad X_{1}=x+i y=\rho e^{i \varphi} .
$$

Уравнение конуса будет $\rho=z$, так что форма

$$
\omega_{0}=d X_{3} \wedge d \log X_{1}=d(i z) \wedge d(\log z+i \varphi)=-d z \wedge d \varphi
$$

совпадает с формой площади цилиндра единичного радиуса.

Переменная $z$ не принимает значения $z=0$, которое соответствует вершине конуса, отсутствующей в орбите $\mathscr{O}_{0}$, однако из приведенных геометрических соображений видно, что если вместо одной точки - вершины “вклеить" окружность - сечение цилиндра плоскостью $z=0$, то получившееся многообразие останется симплектическим, т. е. форма $\omega_{0}$ будет гладкой, всюду отличной от нуля (формой площади всего цилиндра).

На неформальном языке такая вклейка означает допущение к рассмотрению линий

$$
A(t)=\left(\begin{array}{cc}
X_{3}(t) & X_{1}(t) \\
X_{2}(t) & -X_{3}(t)
\end{array}\right) \subset \mathrm{sl}(2, \mathbb{C})
$$

проходящих через нуль, т. е. через $X_{1}(0)=X_{2}(0)=X_{3}(0)=0$, но линии, имеющие разный предел $X_{1}: X_{2}: X_{3}$, в этой точке не будут пересекаться, они будут проходить через разные точки вклеенной "окружности" - проективной прямой $\mathbb{C P}^{1}$ в комплексном случае, или, иными словами, через разные точки проективизации орбиты $\mathscr{O}_{0}$.

Обозначим $\mathbf{P} \operatorname{sl}(2, \mathbb{C})$ проективизацию $\operatorname{sl}(2, \mathbb{C}) \backslash\left(\begin{array}{ll}0 & 0 \\ 0 & 0\end{array}\right)$. Элементы $\mathbf{P} \operatorname{sl}(2, \mathbb{C})$ (временно) будем обозначать заглавными буквами с волной:

$$
\begin{gathered}
\text { если } A=\left(\begin{array}{cc}
X_{3} & X_{1} \\
X_{2} & -X_{3}
\end{array}\right) \neq 0, \text { то } \widetilde{A}=\left(\begin{array}{cc}
x_{3} & x_{1} \\
x_{2} & -x_{3}
\end{array}\right) \in \mathbf{P} \operatorname{sl}(2, \mathbb{C}), \\
\text { где } x_{1}: x_{2}: x_{3}=X_{1}: X_{2}: X_{3} \in \mathbb{C P}^{2} .
\end{gathered}
$$

Обозначим через $\mathbf{P} \mathscr{O}_{R^{2}}$ проективизацию орбиты какого-нибудь ненулевого $A \in$ $\operatorname{sl}(2, \mathbb{C})$; число $\langle A, A\rangle=R^{2}$ параметризует эти многообразия. Если $R^{2} \neq 0$, 
то $\mathbf{P} \mathscr{O}_{R^{2}}$ двумерно; если $R^{2}=0$, то $\mathbf{P} \mathscr{O}_{0}$ одномерно, изоморфно квадрике на $\mathbb{C P}^{2}$ :

$$
\widetilde{A}=\left(\begin{array}{cc}
x_{3} & x_{1} \\
x_{2} & -x_{3}
\end{array}\right) \in \mathbf{P} \mathscr{O}_{0} \quad \Leftrightarrow \quad x_{1} x_{2}+x_{3}^{2}=0, \quad x_{1}: x_{2}: x_{3} \in \mathbb{C P}^{2},
$$

а также изоморфно $\mathbb{C P}^{1} \ni x_{1}: x_{3}=-x_{3}: x_{2}$.

ОПРЕДЕЛЕНИЕ 1 (геометрическое). Зададим симплектическое многообразие $\left(\mathscr{C}_{R^{2}}, \omega_{R^{2}}\right)$ как пару, где $\mathscr{C}_{R^{2}}-$ подмногообразие $\mathrm{sl}(2, \mathbb{C}) \times \mathbf{P} \mathscr{O}_{R^{2}}$, определяемое уравнениями

$$
\left(\left(\begin{array}{cc}
X_{3} & X_{1} \\
X_{2} & -X_{3}
\end{array}\right),\left(\begin{array}{cc}
x_{3} & x_{1} \\
x_{2} & -x_{3}
\end{array}\right)\right) \in \mathscr{C}_{R^{2}} \Leftrightarrow\left\{\begin{array}{l}
X_{1} X_{2}+X_{3}^{2}=\frac{R^{2}}{2} \\
\exists x_{0} \in \mathbb{C}: X_{i}=x_{0} x_{i}
\end{array},\right.
$$

а форма $\omega_{R^{2}}$ имеет вид

$$
\begin{aligned}
\omega_{R^{2}} & =d X_{3} \wedge d \log X_{1}=-d X_{3} \wedge d \log X_{2} \\
& =d\left(x_{3}: x_{1}\right) \wedge d X_{1}=-d\left(x_{3}: x_{2}\right) \wedge d X_{2} .
\end{aligned}
$$

Мы видим, что если $(X, \tilde{Y}) \in \mathscr{C}_{R^{2}}$ и $X \neq 0$, то вторая - проективная часть $\tilde{Y}$ не нужна, она восстанавливается по первой части $X$. При $R^{2} \neq 0$ все $\mathscr{C}_{R^{2}}$ изоморфно орбите $\mathscr{O}_{R^{2}}$ - гладкой квадрике, в определении 1 при $R^{2} \neq 0$ проективная часть оставлена для единообразия, чтобы не давать двух разных определений - для $R^{2} \neq 0$ и $R^{2}=0$.

Пусть теперь $R^{2}=0$. Обозначим через $\pi_{c}$ образ проекции $\mathscr{C}_{R^{2}}$ на первый декартов сомножитель (при $R^{2}=0$ это конус).

Прообраз нуля $\pi_{c}^{-1}\left(\begin{array}{ll}0 & 0 \\ 0 & 0\end{array}\right)$ - гладкое подмногообразие в $\mathscr{C}_{0}=\operatorname{sl}(2, \mathbb{C}) \times \mathbf{P} \mathscr{O}_{0}$, изоморфное многообразию $\mathbf{P} \mathscr{O}_{0}-$ сфере Римана. Пару голоморфных координат, как мы знаем, можно выбрать так:

$$
\begin{gathered}
p, \quad p^{*}=\frac{1}{p}, \quad \text { где } p=x_{3}: x_{1}, p^{*}=-x_{3}: x_{2}, \\
\pi_{c}^{-1}\left(\begin{array}{ll}
0 & 0 \\
0 & 0
\end{array}\right)=\bigcup_{x_{1}: x_{2}: x_{3}}\left(\left(\begin{array}{ll}
0 & 0 \\
0 & 0
\end{array}\right),\left(\begin{array}{cc}
x_{3} & x_{1} \\
x_{2} & -x_{3}
\end{array}\right)\right) .
\end{gathered}
$$

УТВЕРЖДЕНИЕ 4. Все многообразие $\mathscr{C}_{0}$ можно покрыть двумя картами $U$ и $U^{*}$, каждая из которых симплектически изоморфна $\left(\mathbb{C}^{2}, d p \wedge d q\right)$.

ДокАЗАТЕЛЬСтво. Определим отображения $\varphi: \mathbb{C}^{2} \rightarrow U \subset \mathscr{C}_{0}$ и $\varphi^{*}: \mathbb{C}^{2} \rightarrow$ $U^{*} \subset \mathscr{C}_{0}$ так:

$$
\begin{aligned}
\varphi:(p, q) & \mapsto\left(\left(\begin{array}{cc}
p q & q \\
-p^{2} q & -p q
\end{array}\right),\left(\begin{array}{cc}
p & 1 \\
-p^{2} & -p
\end{array}\right)\right) \in \operatorname{sl}(2, \mathbb{C}) \times \mathbf{P} \mathscr{O}_{0}, \\
\varphi^{*}:\left(p^{*}, q^{*}\right) & \mapsto\left(\left(\begin{array}{cc}
-p^{*} q^{*} & -p^{* 2} q^{*} \\
q^{*} & p^{*} q^{*}
\end{array}\right),\left(\begin{array}{cc}
-p^{*} & -p^{* 2} \\
1 & p^{*}
\end{array}\right)\right) \in \operatorname{sl}(2, \mathbb{C}) \times \mathbf{P} \mathscr{O}_{R^{2}} .
\end{aligned}
$$


Если $p p^{*} \neq 0$, то определены функции склейки $p^{*}=1 / p, q^{*}=-p^{2} q$. Очевидно, что $d p \wedge d q=d p^{*} \wedge d q^{*}$, так что многообразие $U \cup U^{*}=\mathscr{C}_{0}$, где

$$
\begin{aligned}
U & =\bigcup_{p, q}\left(\left(\begin{array}{cc}
p q & q \\
-p^{2} q & -p q
\end{array}\right),\left(\begin{array}{cc}
p & 1 \\
p^{2} & -p
\end{array}\right)\right), \\
U^{*} & =\bigcup_{p^{*}, q^{*}}\left(\left(\begin{array}{cc}
-p^{*} q^{*} & -p^{* 2} q^{*} \\
q^{*} & p^{*} q^{*}
\end{array}\right),\left(\begin{array}{cc}
-p^{*} & -p^{* 2} \\
1 & p^{*}
\end{array}\right)\right),
\end{aligned}
$$

является симплектическим. Вне проективной прямой $(q=0, p \in \mathbb{C}) \cup\left(q^{*}=0\right.$, $\left.p^{*} \in \mathbb{C}\right)$ многообразие $\mathscr{C}_{0}$ изоморфно орбите какого-нибудь изотропного ненулевого элемента, например $\sigma_{+}$. Добавленная проективная прямая - раздутие вершины конуса; мы будем называть ее раздутым дивизором.

Группа $\operatorname{SL}(2, \mathbb{C})$ действует и на проективизации орбиты $\mathscr{O}_{0} ;$ в частности, на раздутом дивизоре она действует как группа дробно-линейных преобразований.

ЗАмечАниЕ 10. Такими же картами $U, U^{*}$ можно покрыть и невырожденную квадрику $\mathscr{C}_{R^{2}}, R^{2} \neq 0$ :

$$
\begin{gathered}
\left(\begin{array}{cc}
p q+\sqrt{R^{2} / 2} & q \\
-p\left(p q+2 \sqrt{R^{2} / 2}\right) & -\left(p q+\sqrt{R^{2} / 2}\right)
\end{array}\right) \\
=\left(\begin{array}{cc}
-\left(p^{*} q^{*}+\sqrt{R^{2} / 2}\right) & -p^{*}\left(p^{*} q^{*}+2 \sqrt{R^{2} / 2}\right) \\
q^{*} & p^{*} q^{*}+\sqrt{R^{2} / 2}
\end{array}\right),
\end{gathered}
$$

что позволяет дать определение $\mathscr{C}_{R^{2}}$ как абстрактного многообразия, покрытого двумя картами - $U \ni(p, q)$ и $U^{*} \ni\left(p^{*}, q^{*}\right)$ (так обычно и делается, см. [8]):

ОПРЕдЕлЕниЕ 2 (абстрактное). Зададим симплектическое многообразие $\left(\mathscr{C}_{R^{2}}, \omega_{R^{2}}\right)$ атласом, состоящим из двух карт $\mathbb{C}^{2}$ с координатами $(p, q)$ и $\left(p^{*}, q^{*}\right)$, функциями переклейки $p^{*}=1 / p, q^{*}=-p\left(p q+2 \sqrt{R^{2} / 2}\right)$ и симплектической формой $\omega_{R^{2}}=d p \wedge d q=d p^{*} \wedge d q^{*}$.

ЗАмечАниЕ 11. Хотя в определении и участвует корень $\sqrt{R^{2} / 2}$, результат склейки от выбора знака корня не зависит - многообразия, построенные по $+\sqrt{R^{2} / 2}$ и $-\sqrt{R^{2} / 2}$, изоморфны, это квадрика $(30)$.

Далее многообразие $\mathscr{C}_{R^{2}}$ мы будем называть просто квадрикой (аффинной), подразумевая оговорку, что при $R^{2}=0$ вершина конуса раздута указанным выше образом.

4.2. Гамильтонова (симплектическая) редукция. Гладкие симплектические многообразия $\mathscr{C}_{R^{2}}$ играют важнейшую роль в наших построениях, именно из них мы будем строить фазовое пространство гамильтоновой системы уравнений изомонодромных деформаций (1), которая при $n=3$ является системой Пенлеве VI.

Рассмотрим декартово произведение $n+1$ экземпляра $\left(\mathscr{C}_{a_{i i}}, \omega_{a_{i i}}\right)$, где

$$
a_{i i}=\left\langle A^{(i)}, A^{(i)}\right\rangle=-2 \operatorname{det} A^{(i)} \in \mathbb{C}, \quad i=0,1, \ldots, n,
$$

- параметры нашей задачи (изомонодромной деформации уравнения (1)). 
Прежде чем специальным образом обозначить это пространство, вспомним об оговорке, сделанной во введении (с. 59) - исключим одновременно-треугольные наборы $\Delta_{a_{\bar{i} i}} ;$ обозначим

$$
\widetilde{\widetilde{M}}_{a_{\overline{i i}}}:=\left(\mathscr{C}_{a_{00}} \times \mathscr{C}_{a_{11}} \times \cdots \times \mathscr{C}_{a_{n n}}\right) \backslash \Delta_{a_{\bar{i}}} .
$$

Пространство $\widetilde{\widetilde{M}}_{a_{\bar{i}}}-$ симплектическое (как декартово произведение симплектических пространств), с формой

$$
\widetilde{\widetilde{\omega}}:=\sum_{k=0}^{n} \omega_{a_{k k}}=\sum_{k=0}^{n} d A_{11}^{(k)} \wedge d \log A_{12}^{(k)} .
$$

Выделим в $\widetilde{\widetilde{M}}_{a_{\bar{i}}}$ подмногообразие $\widetilde{M}_{a_{\bar{i}}}$ :

$$
\widetilde{M}_{a_{\bar{i} i}}:=\widetilde{\widetilde{M}}_{a_{\bar{i} \bar{i}}} \cap\left\{\sum_{k=0}^{n} A^{(k)}=0\right\},
$$

состоящее только из тех наборов матриц $A^{(k)}$, для которых $\sum A^{(k)}=0$. Обозначим отображение включения через $\tilde{\tilde{\pi}}: \widetilde{M}_{a_{\overline{i i}}} \hookrightarrow \widetilde{\widetilde{M}}_{a_{\overline{i i}}}$. Ограничение формы $\widetilde{\widetilde{\omega}}$ на $\widetilde{M}_{a_{\bar{i}}}$ обозначим $\widetilde{\omega}:=\tilde{\tilde{\pi}}^{*} \widetilde{\widetilde{\omega}}$.

ЗАмечАниЕ 12 . Многообразие $\left(\widetilde{M}_{a_{\bar{i}}}, \widetilde{\omega}\right)$ не симплектическое, хотя бы потому, что оно нечетномерно - на координаты четномерного (симплектического) многообразия $\widetilde{\widetilde{M}}_{a_{\bar{i}}}$ мы наложили три связи, потребовав равенства нулю трех компонент вектора $\sum A^{(k)}$.

Форма $\widetilde{\omega}$, конечно, остается несингулярной и замкнутой, но иногда она обращается в нуль - те векторы из $T_{A^{(\bar{i})}} \widetilde{\widetilde{M}}_{a_{\overline{i \bar{i}}}}$, на которых $\widetilde{\tilde{\omega}}$ была отлична от нуля, не принадлежат уже $T_{A^{(\bar{i})}} \widetilde{M}_{a_{\bar{i} \bar{i}}} \subset T_{A^{(\bar{i})}} \widetilde{\widetilde{M}}_{a_{\bar{i} \bar{i}}}$.

На $\widetilde{\widetilde{M}}_{a_{\bar{i}}}$ и $\widetilde{M}_{a_{\bar{i}}}$ покомпонентно действует группа $\operatorname{SL}(2, \mathbb{C})$. Для $g \in \operatorname{SL}(2, \mathbb{C})$ положим

$$
g\left(A^{(0)}, \ldots, A^{(n)}\right):=\left(A_{g}^{(0)}, \ldots, A_{g}^{(n)}\right),
$$

где $A_{g}^{(i)}:=g^{-1} A^{(i)} g,-$ такое действие называется диагональным.

Нашей целью является факторизация $\widetilde{M}_{a_{\bar{i}}}$ по этому действию:

$$
M_{a_{\overline{i i}}}:=\widetilde{M}_{a_{\bar{i}}} / \operatorname{SL}(2, \mathbb{C}),
$$

что соответствует отождествлению всевозможных $\Psi\left(P ; P_{0}, \ldots, P_{n}\right)$, связанных калибровочными преобразованиями $\Psi\left(P ; P_{\bar{k}}\right) \sim \Phi\left(P_{\bar{k}}\right) \Psi\left(P ; P_{\bar{k}}\right)$.

Факторизация многообразия, обладающего дополнительной структурой, по действию группы Ли - задача непростая (см. [32; добавление 5], [33], [34] и цитированную там литературу). Мы не будем излагать общую теорию, для этого нужно вводить слишком много не нужных в дальнейшем понятий, а приведем независимые доказательства всех утверждений - реализуем схему гамильтоновой (пуассоновой) редукиии явно - дадим содержательную иллюстрацию общей теории. 
Сначала докажем, что $M_{a_{\bar{i}}}$, множество орбит действия $\operatorname{SL}(2, \mathbb{C})$ на $\widetilde{M}_{a_{\bar{i}}}$, действительно является многообразием. Дадим несколько вспомогательных определений.

Для любого набора элементов $s_{1}, \ldots, s_{N}$ назовем расширенным набор, содержащий как эти элементы, так и всевозможные их коммутаторы: $s_{\bar{k}}, s_{\overline{i j}}$, здесь $s_{i j}:=\left[s_{i}, s_{j}\right]$. Назовем матрищей Грама набора матрицу всевозможных скалярных произведений векторов этого набора.

УТВЕРЖДЕНИЕ 5. Любъе два набора $s_{1}, \ldots, s_{N} u s_{1}^{\prime}, \ldots, s_{N}^{\prime}$, pасширенные наборы которых полны (содержат базис $\mathrm{sl}(2, \mathbb{C}))$, можно совместить преобразованием из $\mathrm{SL}(2, \mathbb{C})$ тогда и только тогда, когда совпадают матриць Грама их расширенных наборов, т.е.

$$
\left\langle s_{i}, s_{j}\right\rangle=\left\langle s_{i}^{\prime}, s_{j}^{\prime}\right\rangle, \quad\left\langle\left[s_{i}, s_{j}\right], s_{k}\right\rangle=\left\langle\left[s_{i}^{\prime}, s_{j}^{\prime}\right], s_{k}^{\prime}\right\rangle \quad \forall i, j, k .
$$

ЗАмечАние 13. Наборы векторов, которые ни сами, ни дополненные коммутаторами не содержат базиса, могут быть приведены $к$ одинаково-треугольному виду (например, верхнетреугольному), и именно эти наборы мы исключили из рассмотрения.

ЗАмЕчАние 14. Квадрат смешанного произведения (ориентированного объема)

$$
\left\langle\left[A^{(i)}, A^{(j)}\right], A^{(k)}\right\rangle^{2}
$$

является многочленом от попарных произведений $\left\langle A^{(i)}, A^{(j)}\right\rangle$ - элементов матрицы Грама (см. формулу (47) ниже):

$$
f_{i j k}^{2}=\left\langle\left[A^{(i)}, A^{(j)}\right], A^{(k)}\right\rangle^{2}=-2 \operatorname{det}\left|\begin{array}{ccc}
a_{i i} & a_{i j} & a_{i k} \\
a_{j i} & a_{j j} & a_{j k} \\
a_{k i} & a_{k j} & a_{k k}
\end{array}\right|,
$$

так что само значение $f_{i j k}$ является только носителем знака - ориентации набора $A^{(i)}, A^{(j)}, A^{(k)}$.

УтвеРжДЕНИЕ 6. Векторы $A, B,[A, B]$ образуют базис в $\operatorname{sl}(2, \mathbb{C})$ тогда и только тогда, когда $\langle[A, B],[A, B]\rangle \neq 0$, m.е. $[A, B]$ не изотропен.

ДокАЗАТЕЛЬСтво. Величина $\langle[A, B], C\rangle$ - ориентированный объем параллелепипеда, построенного на векторах $A, B$ и $C$, равна нулю тогда и только тогда, когда $A, B$ и $C$ линейно зависимы. В нашем случае $C=[A, B]$.

СЛЕДСТВИЕ 1. Линейная зависимость тройки $A, B,[A, B]$ эквивалентна тому, что существует изотропный вектор, ортогональный как $A$, так и $B$, или, что то же, тому, что через $A$ и $B$ проходит изотропная плоскость.

Следующий известный факт, ввиду его важности, мы сформулируем в виде теоремы.

ТЕОРЕма 3. Множество $M_{a_{\bar{i} \bar{i}}}=\widetilde{M}_{a_{\bar{i} \bar{i}}} / \mathrm{SL}(2, \mathbb{C})$ является алгебраическим многообразием. 
ДокАзАтельство. Пусть сначала все векторы $A^{(i)}$ не равны 0, т. е. ни одна вершина не находится на раздутом дивизоре.

Поскольку величины $a_{i j}, f_{i j k}$ инвариантны относительно действия $\operatorname{SL}(2, \mathbb{C})$ на $\widetilde{M}_{a_{\bar{i}}}$, они в то же время являются функциями и на $M_{a_{\bar{i}}}$.

Так как все $A^{(i)}$ не лежат на одной изотропной плоскости, то обязательно найдутся не пропорциональные друг другу $A^{\left(i_{1}\right)}$ и $A^{\left(i_{2}\right)}$ такие, что проходящая через них плоскость не изотропна. В этом случае $A^{\left(i_{1}\right)}, A^{\left(i_{2}\right)},\left[A^{\left(i_{1}\right)}, A^{\left(i_{2}\right)}\right]-$ базис и пока эти векторы линейно независимы, величины $a_{i_{1} i}, a_{i_{2} i}, f_{i_{1} i_{2}}, i=$ $0,1, \ldots, n+1$, фиксируют весь набор $A^{(i)}$ с точностью до действия $\operatorname{SL}(2, \mathbb{C})$.

Величина $f_{i_{1} i_{2} i}$ с точностью до знака определяется значениями $a_{i_{1} i}, a_{i_{2} i}, a_{i_{1} i_{2}}$ и параметрами $a_{j j}$ (формула (35)).

Из элементарно-геометрических соображений понятно, что если

(a) "длины" $a_{i i}$ всех векторов $A^{(i)}$ (включая $A^{\left(i_{1}\right)}$ и $A^{\left(i_{2}\right)}$ ) фиксированы,

(b) заданы скалярные произведения $A^{(i)}$ на "базисные векторы $A^{\left(i_{1}\right)}, A^{\left(i_{2}\right)}$ ", т. е. $2(n-1)$ величин $a_{i_{1} i}, a_{i_{2} i}, i_{1} \neq i \neq i_{2}$,

(c) задан "угол" между $A^{\left(i_{1}\right)}$ и $A^{\left(i_{2}\right)}$ - величина $a_{i_{1} i_{2}}$, то этот набор $2(n-1)+1$ чисел фиксирует все векторы $A^{(j)}$ с точностью до отражения относительно плоскости, содержащей $A^{\left(i_{1}\right)}$ и $A^{\left(i_{2}\right)}$, т. е. с точностью до аргумента величины $f_{i_{1} i_{2} j}=\left\langle\left[A^{\left(i_{1}\right)}, A^{\left(i_{2}\right)}\right], A^{(j)}\right\rangle$ - знака корня из определителя в формуле (35). Добавим $n-1$ величину $f_{i_{1} i_{2}}, i_{1} \neq i \neq i_{2}$, и $n-1$ уравнение $(35)$ на каждую; получившееся подмногообразие пространства $\mathbb{C}^{3(n-1)+1}$ параметризует наборы векторов $A^{(\bar{i})}$ с фиксированными скалярными произведениями на базисные векторы - задает набор $A^{(\bar{i})}$ с точностью до поворота в пространстве - точку $\widetilde{\widetilde{M}}_{a_{\bar{i}}} / \mathrm{SL}(2, \mathbb{C})$. Чтобы получить точку $M_{a_{\bar{i}}}$, осталось выделить из них те, у которых сумма всех матриц равна нулю.

Мы доказали, что $M_{a_{\bar{i}}}$ - алгебраическое многообразие, изоморфное $2(n-2)$ мерному подмногообразию $M_{a_{\bar{i}}}^{f}$, выделяемому в $\mathbb{C}^{3 n-2} n+2$ алгебраическими уравнениями

$$
\left\{\begin{array}{l}
f_{i_{1} i_{2} i}^{2}=-2 \operatorname{det}\left|\begin{array}{lll}
a_{i_{1} i_{1}} & a_{i_{1} i_{2}} & a_{i_{1} i} \\
a_{i_{2} i_{1}} & a_{i_{2} i_{2}} & a_{i_{2} i} \\
a_{i i_{1}} & a_{i i_{2}} & a_{i i}
\end{array}\right|, \quad i_{1} \neq i \neq i_{2}, \\
\sum_{i=0}^{n} a_{i_{1} i}=0, \quad \sum_{i=0}^{n} a_{i_{2} i}=0, \quad \sum_{i=0}^{n} f_{i_{1} i_{2} i}=0 .
\end{array}\right.
$$

Пусть теперь среди $A^{(i)}$ есть нулевые - точки с раздутых дивизоров. Точке $\left(0, \widetilde{A}^{\left(j_{0}\right)}\right)$ с $\mathscr{O}_{0}$ взаимно однозначно соответствует изотропное направление в $\operatorname{sl}(2, \mathbb{C})$. Такие направления параметризуются точками проективной прямой значением отношений

$$
\left\langle\widetilde{A}^{\left(j_{0}\right)}, A^{\left(i_{1}\right)}\right\rangle:\left\langle\widetilde{A}^{\left(j_{0}\right)}, A^{\left(i_{2}\right)}\right\rangle:\left\langle\widetilde{A}^{\left(j_{0}\right)},\left[A^{\left(i_{1}\right)}, \widetilde{A}^{\left(i_{2}\right)}\right]\right\rangle
$$

при одном условии - $\widetilde{A}^{\left(j_{0}\right)}$ должно быть с квадрики $\left\langle\widetilde{A}^{\left(j_{0}\right)}, \widetilde{A}^{\left(i_{0}\right)}\right\rangle=0$.

На $2(n-2)$-мерном многообразии коэффициентов $a_{i_{1} i}, a_{i_{2} i}\left(f_{i_{1} i_{2} i}^{2}\right.$ по ним определяется) подмногообразие $a_{i_{1} j_{0}}=a_{i_{2} j_{0}}=0$ коразмерности 2 (мы зафиксировали две координаты) раздувается до коразмерности 1. 
То, что результат будет гладким многообразием, мы увидим в п. 5.4.

ЗАмЕчАниЕ 15 . В случае Пенлеве $n=3$ уравнения

$$
a_{i i}=\text { const }, \quad \sum_{i=0}^{n} a_{i j}=0 \quad \forall j
$$

легко разрешаются относительно любых двух $a_{i j}$ с единственным общим индексом, например $a_{12}$ и $a_{13}$ :

$$
\begin{aligned}
-a_{32} & =a_{12}+a_{13}+\frac{a_{11}+a_{22}+a_{33}-a_{00}}{2}, \\
a_{0 k} & =a_{i j}+\frac{a_{i i}+a_{j j}-a_{00}-a_{k k}}{2},
\end{aligned}
$$

все индексы $i, j, k$ различны и отличны от нуля.

УТвеРЖДЕНИЕ 7. В случае Пенлеве многообразие $M_{a_{\overline{i i}}}$ бирационалъно изоморбно (а если все $a_{i i} \neq 0$, то и просто изоморфно) алгебрачческой поверхности $M_{a_{\overline{i ̆}}}^{f}$, выделяемой в $\mathbb{C}^{4}$ пересечением кубики и гиперплоскости:

$$
\begin{gathered}
f^{2}=-2 \operatorname{det}\left|\begin{array}{ccc}
a_{11} & x & y \\
x & a_{22} & z \\
y & z & a_{33}
\end{array}\right|, \\
x+y+z=\frac{a_{00}-a_{11}-a_{22}-a_{33}}{2}=\mathrm{const}, \\
x=a_{12}, \quad y=a_{13}, \quad z=a_{23}, \quad f=f_{123} .
\end{gathered}
$$

Если среди параметров имеются равные нулю: $a_{j_{0} j_{0}}=0$, то $M_{a_{\bar{i}}}$ изоморфно $M_{a_{\bar{i}}}^{f}$ с раздутыми точками, соответствующими $0=a_{i j_{0}}=f$ для любого $i$.

Для завершения построения симплектического многообразия $\left(M_{a_{\bar{i}}}, \omega\right)$ нужно построить форму $\omega \in \bigwedge^{2} M_{a_{\bar{i}}}$ :

а) доказать, что форма $\widetilde{\omega}$ проектируется с $\widetilde{M}_{a_{\bar{i} \bar{i}}}$ на $M_{a_{\bar{i} \bar{i}}}$, или, иными словами, доказать, что $\widetilde{\omega} \in \bigwedge^{2} \widetilde{M}_{a_{\bar{i}}}$ является поднятием некоторой формы $\omega$ с $M_{a_{\bar{i}}}: \widetilde{\omega}=\tilde{\pi}^{*} \omega$

б) доказать отсутствие нулей у формы $\omega$, а также ее несингулярность и замкнутость (два последних свойства очевидны, так как $\widetilde{\omega}$ замкнута и несингулярна).

Пусть на многообразии $M$ с координатами $x^{i}$ действует группа Ли $G$. Обозначим $A \rightarrow A_{g}, A \in M, g \in G$, действие группы на точки многообразия, $x_{g}^{i}-$ действие элемента группы $g \in G$ на координатные функции:

$$
x_{g}^{i}=x_{g}^{i}(A):=x^{i}\left(A_{g}\right) .
$$

Пусть $w_{i j}$ - коэффициенты разложения какой-нибудь 2-формы $\widetilde{\omega}$ по базисным формам $d x^{i} \wedge d x^{j}$.

Лемма 1. Если форма $\widetilde{\omega}=\sum_{i, j} w_{i j} d x^{i} \wedge d x^{j}$ обладает свойством

$$
\sum_{i, j} w_{i j}\left(A_{g(t)}\right) d x_{g(t)}^{i} \wedge d x_{g(t)}^{j}=\sum_{i, j} w_{i j}(A) d x^{i} \wedge d x^{j}
$$


для любой однопараметрической подгруппљ $G: g(t): \mathbb{R} \rightarrow G$, то

1) борма $\widetilde{\omega}$ является $G$-инвариантной: $g^{*} \widetilde{\omega}=\widetilde{\omega}$ для любого $g \in G$;

2) $\widetilde{\omega}\left(\xi_{0}, \xi\right)=0$ для любого $\xi \in T_{A} M$ и любого вектора $\xi_{0}$ вида

$$
\xi_{0}=\left.\frac{d}{d t}\right|_{t=0} A_{g(t)}
$$

где $A \in M-$ точка $M$ (не зависит от параметра $t$ !), $g(t)$ - произвольная кривая, проходящая при $t=0$ через единичу: $g(0)=\mathrm{id} \in G$.

Прежде чем доказывать утверждения леммы, поясним смысл равенства (40). Кривая $\mathbb{R} \rightarrow G$ порождает на $M$ поток $A(t)=A_{g(t)}: M \times \mathbb{R} \rightarrow M$. Дифференциал $d x_{g(t)}^{i}$ - это функция (форма) на $T^{*}(M \times \mathbb{R})$. При любом $t=t_{0}$ она имеет вид $d x_{g\left(t_{0}\right)}^{i}+\dot{x}_{g\left(t_{0}\right)}^{i} d t$, где $x_{g\left(t_{0}\right)}^{i}$ - функция на $M$, полученная сдвигом аргумента $x^{i}$, сдвигом элементом группы $g\left(t_{0}\right) \in G$, а $\dot{x}_{g\left(t_{0}\right)}^{i}$ - функция на $M$, равная скорости изменения значения $x^{i}$ в точке $A\left(t_{0}\right)$ при движении $A$ по своей траектории $\bigcup_{t} A(t)$.

Равенство (40) требует, в частности, чтобы соответствующая сумма не содержала членов с $d t$ - обращалась бы в нуль на любой паре векторов вида $\left(\partial_{t}, \xi\right)$, $\xi \in T M, \partial_{t} \in T \mathbb{R}$.

Вычисление покажет, что этого достаточно для зануления $\omega\left(\xi_{0}, \xi\right)$ на любом векторе $\xi_{0}$, касательном к траектории $\bigcup_{t} A_{g(t)}$, вне зависимости от второго аргумента $\xi$.

ДокАЗАТЕЛЬСтво ЛЕммы 1. Зафиксируем какую-нибудь точку $A \in M$ и кривую $g(t)$; положим

$$
\xi_{0}=\left.\frac{d}{d t}\right|_{t=0} A_{g(t)} \in T_{A_{g(0)}} M .
$$

Обозначим через $\dot{x}_{g(0)}^{i}$ координаты вектора $\xi_{0}$ :

$$
\dot{x}_{g(0)}^{i}=\left.\frac{d}{d t}\right|_{t=0} x_{g(t)}^{i}=\left.\frac{d}{d t}\right|_{t=0} x^{i}\left(A_{g(t)}\right) .
$$

Тогда

$$
\begin{aligned}
\left.\sum_{i, j} w_{i j}\left(A_{g(t)}\right) d x_{g(t)}^{i} \wedge d x_{g(t)}^{j}\right|_{t=0}= & \sum_{i, j} w_{i j}\left(A_{g(0)}\right)\left(d x_{g(0)}^{i} \wedge d x_{g(0)}^{j}\right. \\
& \left.+\dot{x}_{g(0)}^{i} d t \wedge d x_{g(0)}^{j}+\dot{x}_{g(0)}^{j} d x_{g(0)}^{i} \wedge d t\right) .
\end{aligned}
$$

Из равенства (40) следует, во-первых, что

$$
\sum_{i, j} w_{i j}\left(A_{g(0)}\right) d x_{g(0)}^{i} \wedge d x_{g(0)}^{j}=\sum_{i, j} w_{i j}(A) d x^{i} \wedge d x^{j},
$$

т. е. $G$-инвариантность формы $\widetilde{\omega}$, и, кроме того, что

$$
\begin{aligned}
0 & =d t \wedge \sum_{k, j} w_{k j}\left(A_{g(0)}\right)\left(\dot{x}_{g(0)}^{k} d x_{g(0)}^{j}-\dot{x}_{g(0)}^{j} d x_{g(0)}^{k}\right) \\
& =d t \wedge \widetilde{\omega}\left(A_{g(0)}\right)\left(\xi_{0}, \cdot\right)=d t \wedge i_{\xi_{0}} \widetilde{\omega}\left(A_{g(0)}\right),
\end{aligned}
$$


т. е. значение $\widetilde{\omega}\left(A_{g(0)}\right)\left(\xi_{0}, \cdot\right)$ равно нулю вне зависимости от второго аргумента от вектора, на котором вычисляется значение 1-формы $i_{\xi_{0}} \widetilde{\omega}\left(A_{g(0)}\right)$. Здесь $i_{\xi} \omega$ это 1-форма, внутреннее произведение $\xi$ на 2-форму $\omega$ (см., например, [32]).

TEOPEмA 4. Форма $\widetilde{\omega}$ проектируется с $\widetilde{M}_{a_{\bar{i} \bar{i}}}$ на $M_{a_{\bar{i}}}$.

ДокАЗАТЕЛЬСтво. Рассмотрим линейное отображение - проекцию пространств $\tilde{\pi}: T_{A^{(\bar{k})}} \tilde{M}_{a_{\bar{i} \bar{i}}} \rightarrow T_{\tilde{\pi}^{\prime} A^{(\bar{k})}}\left(\widetilde{M}_{a_{\bar{i}}} / \mathrm{SL}(2, \mathbb{C})\right)$. Его ядром являются векторы, касательные к траекториям, полученным "вращением" постоянных наборов $A^{(\bar{k})}$ :

$$
\xi_{0} \in \operatorname{ker} \tilde{\pi} \quad \Leftrightarrow \quad \exists g(t): \xi_{0}=\left.\frac{d}{d t}\right|_{t=0} A_{g(t)}^{(\bar{k})}, \quad g(0)=\mathrm{id} \in \operatorname{SL}(2, \mathbb{C}) .
$$

Проверим равенство (40) для подгрупп $T_{\text {diag }}^{\mu}, T_{\triangle \downarrow}^{\nu}, T_{\triangle \uparrow}^{\nu}$ диагональных, нижне- и верхнетреугольных матриц.

Так как

$$
\widetilde{\omega}=\sum_{i=0}^{n} d A_{11}^{(i)} \wedge d \log A_{12}^{(i)}
$$

TO

$$
T_{\text {diag }}^{\mu}\left(\begin{array}{cc}
A_{11} & A_{12} \\
A_{21} & -A_{11}
\end{array}\right)=\left(\begin{array}{cc}
A_{11} & A_{12} \mu^{2} \\
A_{21} \mu^{-2} & -A_{11}
\end{array}\right)
$$

и, следовательно,

$$
\sum_{i=0}^{n} d A_{11}^{(i)} \wedge d \log \mu^{-2} A_{11}^{(i)}=\widetilde{\omega}+\left(\sum_{i=0}^{n} d A_{11}^{(i)}\right) \wedge d \log \mu^{2}=\widetilde{\omega} .
$$

Теперь $T_{\triangle \downarrow}^{\nu}$ :

$$
T_{\triangle \downarrow}^{\nu}\left(\begin{array}{cc}
A_{11} & A_{12} \\
A_{21} & -A_{11}
\end{array}\right)=\left(\begin{array}{cc}
A_{11}-\nu A_{12} & A_{12} \\
A_{21}+2 \nu A_{11}-\nu^{2} A_{12} & -A_{11}+\nu A_{12}
\end{array}\right) .
$$

Подставив, получим искомое

$$
\sum_{i=0}^{n} d\left(A_{11}^{(i)}-\nu A_{12}^{(i)}\right) \wedge d \log A_{12}^{(i)}=\widetilde{\omega}-\sum_{i=0}^{n} d \nu \wedge d A_{12}^{(i)}=\widetilde{\omega} .
$$

Вследствие равенства

$$
d A_{11}^{(i)} \wedge d \log A_{12}^{(i)}=-d A_{11}^{(i)} \wedge d \log A_{21}^{(i)}
$$

проверка для подгруппы $T_{\triangle \uparrow}^{\nu}$ аналогична.

Равенства (41), (42) верны для любой зависимости $\mu=\mu(t), \nu=\nu(t)$, поскольку

$$
\sum_{i} d A_{11}^{(i)}=\sum_{i} d A_{12}^{(i)}=\sum_{i} d A_{21}^{(i)}=0 .
$$

Из леммы 1 следует, что $\widetilde{\omega}\left(\xi_{0}, \xi\right)=0$, если $\xi$ - касательный вектор к одной из трех подгрупп $T_{\triangle \downarrow}^{\nu}, T_{\text {diag }}^{\mu}, T_{\triangle \uparrow}^{\nu}$. Касательные к этим трем подгруппам образуют базис касательного пространства, следовательно, $\widetilde{\omega}\left(\xi_{0}, \xi\right)=0$ для любого $\xi$. 
СлеДСтвиЕ 2. Значение суммы (33) не зависит от того, в каком базисе $\operatorname{sl}(2, \mathbb{C})$ вычисляются матричные элементы $A^{(k)}$, этот базис может, например, произвольно зависеть от самих $A^{(k)}$.

ЗАмечание 16. Равенств (41), (42) показывают, почему пришлось переходить от $\widetilde{\widetilde{M}}_{a_{\bar{i}}}$ к $\widetilde{M}_{a_{\bar{i}}}$. Со всего пространства $\widetilde{\widetilde{M}}_{a_{\bar{i}}}$ форма $\widetilde{\widetilde{\omega}}$ на $M_{a_{\bar{i}}}$ не опускается.

Отсутствие нулей у $\omega$ докажем позже (на с. 97), построив покрытие $M_{a_{\bar{i}}}$ симплектическими картами.

Подведем итог. Мы построили симплектическое многообразие $M_{a_{\bar{i}}}$. Вне точек раздутых дивизоров (считаем, что $\left.\mathscr{C}_{a_{k k}} \subset \mathrm{sl}(2, \mathbb{C})\right)$ его связь со стандартным пуассоновым пространством $\mathrm{sl}^{*}(2, \mathbb{C}) \simeq \operatorname{sl}(2, \mathbb{C})$ проиллюстрируем диаграммой

$$
\begin{aligned}
& \operatorname{sl}(2, \mathbb{C})^{n+1} \hookleftarrow \mathscr{C}_{a_{00}} \times \cdots \times \mathscr{C}_{a_{n n}}=\widetilde{\widetilde{M}}_{a_{\bar{i}}} \stackrel{\tilde{\tilde{\pi}}}{\longleftarrow} \widetilde{M}_{a_{\bar{i}}} \stackrel{\tilde{\pi}}{\rightarrow} \tilde{M}_{a_{\bar{i}}} / \operatorname{SL}(2, \mathbb{C})=M_{a_{\bar{i}}} \\
& \{\cdot, \cdot\}_{\mathrm{LP}} \leftarrow \quad\{\widetilde{\sim} \cdot \cdot\} \stackrel{\sim}{\leftrightarrow} \widetilde{\widetilde{\omega}} \quad \stackrel{\tilde{\pi}^{*}}{\rightarrow} \widetilde{\omega} \stackrel{\tilde{\pi}^{*}}{\leftarrow} \omega \in \Lambda^{2} T^{*} M_{a_{\bar{i}}}
\end{aligned}
$$

Здесь символически обозначены: $\{\cdot, \cdot\}_{\mathrm{LP}}$ - скобка Ли-Пуассона на декартовой степени $\operatorname{sl}(2, \mathbb{C}) \simeq \operatorname{sl}^{*}(2, \mathbb{C}),\{\stackrel{\approx}{*} \cdot \cdot\}$ - скобка Пуассона $\sum \Pi^{i j} \frac{\partial}{\partial x^{i}} \frac{\partial}{\partial x^{j}}$, канонически соответствующая симплектической форме $\widetilde{\widetilde{\omega}}=\sum \Pi_{i j} d x^{i} \wedge d x^{j}$; это каноническое соответствие обозначено “㕕”.

Умножим теперь каждое из пространств цепочки

$$
\operatorname{sl}(2, \mathbb{C})^{n+1} \hookleftarrow \widetilde{M}_{a_{\bar{i} i}} \hookleftarrow \widetilde{M}_{a_{\bar{i} i}} \longrightarrow M_{a_{\bar{i} \bar{i}}}
$$

декартово на $\Lambda$ - рассмотрим расширенные фазовые пространства. Будем обозначать расширенные пространства той же буквой, но с индексом $\Lambda$ :

$$
\widetilde{M}_{a_{\bar{i} \bar{i}}}^{\Lambda}:=\widetilde{M}_{a_{\bar{i} \bar{i}}} \times \Lambda, \quad M_{a_{\bar{i} \bar{i}}}^{\Lambda}:=M_{a_{\bar{i} \bar{i}}} \times \Lambda .
$$

Все уравнения вида $(1)$, являющиеся прообразом какой-нибудь точки $M_{a_{\bar{i}}}$, имеют одинаковые монодромии, следовательно, действие калибровочной группы $\Psi(\lambda) \rightarrow \Phi_{l} \Psi(\lambda), A^{(k)} \rightarrow \Phi_{l}^{-1} A^{(k)} \Phi_{l}$ монодромии не меняет, и условие постоянства набора данных монодромии корректно определено и на $M_{a_{\bar{i}}}$, задает его (изомонодромное) слоение.

Листы слоения - образы листов слоения Шлезингера (15), а также любого другого, отличающегося выбором $R$, например как в (17). Таким образом, 2 -форма $\Omega_{\mathrm{PVI}}:=\omega-d h$ определяет интегрируемое распределение и на $M_{a_{\bar{i} i}}^{\Lambda}$ :

$$
\Omega_{\mathrm{PVI}}:=\omega-d h=\omega-\sum_{i<j} d a_{i j} \wedge d \log \left(\lambda_{i}-\lambda_{j}\right) .
$$

Мы больше не пишем $\pi_{M}^{*}$ (формулы (19), (21)), которое лишь напоминало, что $\omega$ поднята с $M_{a_{\overline{i i}}}$ на $M_{a_{\bar{i}}}^{\Lambda}$, считаем, что $\omega$ задана сразу на $M_{a_{\overline{i i}}}^{\Lambda}$.

Как зависят листы изомонодромного слоения от параметров $\lambda_{k}$ ? Из самой постановки задачи изомонодромной деформации (см. с. 63) следует, что если 
какая-нибудь деформация $\Psi\left(\lambda ; \lambda_{0}, \ldots, \lambda_{n}\right)$ изомонодромна, то и деформация $\Psi\left(\lambda^{\prime} ; \lambda_{0}^{\prime}, \ldots, \lambda_{n}^{\prime}\right)$ изомонодромна, где

$$
\lambda_{i}^{\prime}:=\frac{a_{1} \lambda_{i}+a_{2}}{a_{3} \lambda_{i}+a_{4}}, \quad a_{k}=a_{k}\left(\lambda_{0}, \ldots, \lambda_{n}\right),
$$

- произвольное движение сферы Римана $\mathbb{C P}^{1} \ni \lambda_{i}$. Это так, потому что ни вычеты в полюсах $d_{\downarrow} \Psi \Psi^{-1}$, ни появляющиеся у $\Psi(\lambda)$ при эволюции по замкнутым циклам множители монодромии не зависят от параметризации $\lambda$ сферы Римана. Таким образом, получаем следствие.

СлеДСТВИЕ 3. Интегралъные поверхности уравнений изомонодромных деформачий (43) в пространстве $M_{a_{i i}}^{\Lambda}$ переходят друг в друга при произволвньх дробно-линейных преобразованиях системы параметров $\lambda_{i}$ :

$$
\begin{gathered}
\left(\lambda_{0}, \ldots, \lambda_{n}\right) \mapsto\left(\lambda_{0}^{\prime}, \ldots, \lambda_{n}^{\prime}\right), \quad \lambda_{i}^{\prime}=\frac{a_{1} \lambda_{i}+a_{2}}{a_{3} \lambda_{i}+a_{4}}, \\
\left(\begin{array}{ll}
a_{1} & a_{2} \\
a_{3} & a_{4}
\end{array}\right) \in \mathrm{GL}(2, \mathbb{C}), \quad a_{k}=a_{k}\left(\lambda_{0}, \ldots, \lambda_{n}\right) .
\end{gathered}
$$

ЗАмечАниЕ 17. Система Шлезингера (15) не обладает этим свойством, она не инвариантна относительно инверсии.

Итак, уравнения, заданные формой (43), инвариантны как относительно одновременного сопряжения всех $A^{(i)}$ произвольной матрицей, так и относительно движений сферы Римана, на которой лежат параметры деформации уравнения (1).

ЗАмЕчАНИЕ 18. Поскольку уравнение (43) не зависит от дробно-линейных преобразований, то можно зафиксировать какие-нибудь три значения $\lambda_{i}$, например положить

$$
\lambda_{0}=0, \quad \lambda_{n-1}=1, \quad \lambda_{n}=\infty ;
$$

тогда остальные значения $\lambda_{i}, i=1, \ldots, n-2$, будут независимыми комплексными параметрами - "временами".

Другими словами, в качестве нетривиальных "времен” можно выбрать $n-2$ двойных отношения

$$
\hat{t}_{k}:=\mathscr{D}\left(\lambda_{n-1}, \lambda_{k}, \lambda_{n}, \lambda_{0}\right)=\lambda_{k}, \quad k=1, \ldots, n .
$$

Здесь и далее двойное отношение обозначено буквой $\mathscr{D}$ :

$$
\mathscr{D}(A, B, C, D):=\frac{(A-C)(B-D)}{(A-D)(B-C)} .
$$

\section{5. Построение рациональных координат Дарбу на $M_{a_{\overline{i i}}}$}

Пространство $M_{a_{\bar{i}}}$ определено как фактор $\widetilde{M}_{a_{\overline{i i}}} / \mathrm{SL}(2, \mathbb{C})$. Геометрической основой доказательства того, что в результате факторизации получился "хороший” (гладкий, аналитический) объект - алгебраическое многообразие, было 
следующее наблюдение: два полных (т.е. содержащих базис) набора векторов переводятся друг в друга ортогональным преобразованием тогда и только тогда, когда совпадают их матрицы Грама (матрицы попарных скалярных произведений).

В этом разделе будем эксплуатировать другую геометрическую идею.

Выделим среди всевозможных базисов линейного пространства (у нас это $\operatorname{sl}(2, \mathbb{C}))$ подкласс базисов, состоящий из какого-нибудь одного выделенного базиса и полученных из него действием всевозможных элементов какой-нибудь группы (в нашем случае - $\mathrm{SL}(2, \mathbb{C}))$, т. е. рассмотрим орбиту действия группы $\mathrm{SL}(2, \mathbb{C})$ в многообразии базисов $\operatorname{sl}(2, \mathbb{C})$. Назовем базисы этого подкласса стандартными.

Пусть каким-нибудь (однозначным, вполне определенным) образом по набору векторов (у нас - по набору матриц-вычетов $A^{(0)}, \ldots, A^{(n)}$ ) удается строить стандартный базис; иными словами, пусть задано отображение множества наборов $A^{(\bar{i})}$ в множество стандартных базисов (пространства $\mathrm{sl}(2, \mathbb{C})$ ). Назовем такой, определенным образом построенный по набору $A^{(i)}$, стандартный базис сопутствующим (набору $A^{(\bar{i})}$ ). Координаты векторов набора $A^{(\bar{i})}$ в стандартном сопутствующем базисе являются функциями на факторпространстве $\widetilde{M}_{a_{\bar{i}}} / \mathrm{SL}(2, \mathbb{C})$, из них можно выбрать (составить) набор координатных функций многообразия $M_{a_{\bar{i}}}$.

Пусть $\sigma^{(\bar{i})}=\sigma^{(1)}, \sigma^{(2)}, \sigma^{(3)}$ - произвольный базис $\mathrm{sl}(2, \mathbb{C})$ и $\sigma_{g}^{(\bar{i})}=g^{-1} \sigma^{(1)} g$, $g^{-1} \sigma^{(2)} g, g^{-1} \sigma^{(3)} g-$ он же, "повернутый” элементом $g \in \operatorname{SL}(2, \mathbb{C}) ;$ пусть

$$
\mathrm{SL} \sigma^{(\bar{i})}:=\bigcup_{g \in \operatorname{SL}(2, \mathbb{C})} \sigma_{g}^{(\bar{i})}
$$

- орбита действия $\mathrm{SL}(2, \mathbb{C})$ на $\sigma^{(\bar{i})}$, и пусть построено отображение

$$
\widetilde{M}_{a_{\bar{i} \bar{i}}} \rightarrow \mathrm{SL} \sigma^{(\bar{i})}: A^{(\bar{i})} \mapsto \sigma^{(1)}\left(A^{(\bar{i})}\right), \sigma^{(2)}\left(A^{(\bar{i})}\right), \sigma^{(3)}\left(A^{(\bar{i})}\right),
$$

коммутирующее с действием $\mathrm{SL}(2, \mathbb{C})$ :

$$
\sigma^{(1)}\left(A_{g}^{(\bar{i})}\right), \sigma^{(2)}\left(A_{g}^{(\bar{i})}\right), \sigma^{(3)}\left(A_{g}^{(\bar{i})}\right)=\sigma_{g}^{(1)}\left(A^{(\bar{i})}\right), \sigma_{g}^{(2)}\left(A^{(\bar{i})}\right), \sigma_{g}^{(3)}\left(A^{(\bar{i})}\right) .
$$

Назовем это свойство $\mathrm{SL}(2, \mathbb{C})$-инвариантностъю.

УТВеРЖДЕниЕ 8. Координаты (матричные элементы) $A_{i j}^{(k)} \in \operatorname{sl}(2, \mathbb{C})$ в базисе $\sigma^{(\bar{i})}\left(A^{(\bar{i})}\right)$, построенном $\mathrm{SL}(2, \mathbb{C})$-инвариантно, не зависят от действия $\mathrm{SL}(2, \mathbb{C})$ на $\widetilde{M}_{a_{\bar{i}}}$, они являются функииями на $M_{a_{\bar{i}}}=\widetilde{M}_{a_{\bar{i}}} / \mathrm{SL}(2, \mathbb{C})$.

ЗАмЕчАНИЕ 19. Примером построения такого базиса является процедура ортонормирования, когда первый базисный вектор выбирается единичным сонаправленным с первым вектором набора, следующий базисный вектор единичный вектор из полуплоскости, содержащей первый и второй векторы и т. д.

Утверждается, что два набора векторов в $\mathbb{R}^{n}$ можно перевести друг в друга ортогональным преобразованием (т. е. при факторизации по $\mathrm{O}(n)$ такие наборы дадут одну точку) тогда и только тогда, когда в так построенных ортонормированных базисах векторы обоих наборов имеют одинаковые координаты. 
Эти, общие для всех наборов, значения являются функциями на фактормногообразии, из них можно составить координатный набор. Мы, однако, будем использовать другой метод, основанный на свойствах коммутатора в $\operatorname{sl}(2, \mathbb{C})$.

5.1. Аналитическая геометрия в терминах $\mathrm{sl}(2, \mathbb{C})$.

ОПРЕДЕЛЕНИЕ 3. Назовем стандартным любой базис $\operatorname{sl}(2, \mathbb{C})$, получающийся из

$$
\sigma_{3}=\left(\begin{array}{cc}
1 & 0 \\
0 & -1
\end{array}\right), \quad \sigma_{+}=\left(\begin{array}{ll}
0 & 1 \\
0 & 0
\end{array}\right), \quad \sigma_{-}=\left(\begin{array}{ll}
0 & 0 \\
1 & 0
\end{array}\right)
$$

действием $\mathrm{SL}(2, \mathbb{C})$, т. е. удовлетворяющий равенствам

$$
\begin{aligned}
\left\langle\sigma_{3}, \frac{\sigma_{3}}{2}\right\rangle & =\left\langle\sigma_{+}, \sigma_{-}\right\rangle=1, \\
{\left[\sigma_{3}, \sigma_{ \pm}\right] } & = \pm 2 \sigma_{ \pm} \\
\left\langle\sigma_{+}, \sigma_{+}\right\rangle & =\left\langle\sigma_{-}, \sigma_{-}\right\rangle=\left\langle\sigma_{3}, \sigma_{+}\right\rangle=\left\langle\sigma_{3}, \sigma_{-}\right\rangle=0 .
\end{aligned}
$$

ЗАмЕчАниЕ 20. Такие условия “стандартности” базиса очевидным образом связаны с другим условием, однозначно фиксирующим поворот и ориентацию евклидова пространства, - ортонормированностью и правой ориентацией.

Определим евклидово скалярное произведение на $\mathrm{sl}(2, \mathbb{C})$ формулой

$$
\langle A, B\rangle_{\mathrm{e}}:=-2 \operatorname{tr} A B=-2\langle A, B\rangle,
$$

векторное же произведение оставим как и было - коммутатором $[A B]=$ $A B-B A$. Легко видеть, что относительно $\langle\cdot, \cdot\rangle_{\mathrm{e}}$ и $[\cdot, \cdot]$ базис $\vec{e}_{k}:=\frac{\sqrt{-1}}{2} \sigma_{k}$, где

$$
\sigma_{1}=\left(\begin{array}{ll}
0 & 1 \\
1 & 0
\end{array}\right), \quad \sigma_{2}=\left(\begin{array}{cc}
0 & \sqrt{-1} \\
-\sqrt{-1} & 0
\end{array}\right), \quad \sigma_{3}=\left(\begin{array}{cc}
1 & 0 \\
0 & -1
\end{array}\right)
$$

является правым ортонормированным. Раскладывая по этому базису произвольные векторы $A, B, C \in \operatorname{sl}(2, \mathbb{C})$, запишем основные формулы аналитической геометрии в $\operatorname{sl}(2, \mathbb{C})$-версии:

$$
\begin{gathered}
{[A,[B, C]]=B\langle A, C\rangle_{\mathrm{e}}-C\langle A, B\rangle_{\mathrm{e}},} \\
\langle A,[B, C]\rangle_{\mathrm{e}}=\operatorname{det} \mathscr{G}, \quad \mathscr{G}:=\left(\begin{array}{lll}
a_{1} & a_{2} & a_{3} \\
b_{1} & b_{2} & b_{3} \\
c_{1} & c_{2} & c_{3}
\end{array}\right),
\end{gathered}
$$

где

$$
A=\sum a_{i} \vec{e}_{i}, \quad B=\sum b_{i} \vec{e}_{i}, \quad C=\sum c_{i} \vec{e}_{i}
$$

Отсюда следует, что

$$
\langle A,[B, C]\rangle_{\mathrm{e}}^{2}=\operatorname{det} \mathscr{G} \operatorname{det} \mathscr{G}=\operatorname{det} \mathscr{G} \mathscr{G}^{T}=\operatorname{det}\left|\begin{array}{ccc}
\langle A, A\rangle_{\mathrm{e}} & \langle A, B\rangle_{\mathrm{e}} & \langle A, C\rangle_{\mathrm{e}} \\
\langle B, A\rangle_{\mathrm{e}} & \langle B, B\rangle_{\mathrm{e}} & \langle B, C\rangle_{\mathrm{e}} \\
\langle C, A\rangle_{\mathrm{e}} & \langle C, B\rangle_{\mathrm{e}} & \langle C, C\rangle_{\mathrm{e}}
\end{array}\right|
$$


- многочлен от элементов матрицы Грама набора $A, B, C$. Перепишем эти формулы, используя произведение Киллинга $\langle\cdot, \cdot\rangle=-\frac{1}{2}\langle\cdot, \cdot\rangle_{\mathrm{e}}$ :

$$
-\frac{1}{2}[A,[B, C]]=B\langle A, C\rangle-C\langle A, B\rangle .
$$

Умножим скалярно на $D$ и учтем, что при циклической перестановке строк определитель $3 \times 3$ не меняется, $\langle A,[B, C]\rangle=\langle C,[A, B]\rangle$ :

$$
\begin{aligned}
-\frac{1}{2}\langle[A, B],[C, D]\rangle & =\langle A, C\rangle\langle B, D\rangle-\langle A, D\rangle\langle B, C\rangle, \\
-\frac{1}{2}\langle A,[B, C]\rangle^{2} & =\operatorname{det}\left|\begin{array}{ccc}
\langle A, A\rangle & \langle A, B\rangle & \langle A, C\rangle \\
\langle B, A\rangle & \langle B, B\rangle & \langle B, C\rangle \\
\langle C, A\rangle & \langle C, B\rangle & \langle C, C\rangle
\end{array}\right| .
\end{aligned}
$$

Аналогом формулы разложения по ортонормированному базису $\vec{e}_{i}$,

$$
A=\sum_{k}\left\langle A, \vec{e}_{k}\right\rangle_{\mathrm{e}} \vec{e}_{k}
$$

является следующая формула разложения по стандартному базису:

$$
A=\sigma_{3}\left\langle A, \frac{\sigma_{3}}{2}\right\rangle+\sigma_{+}\left\langle A, \sigma_{-}\right\rangle+\sigma_{-}\left\langle A, \sigma_{+}\right\rangle
$$

- сразу видно, что обе части равенства имеют до́лжные скалярные произведения на базисные векторы $\sigma_{3}, \sigma_{+}, \sigma_{-}$. Обозначим коэффициенты разложения по стандартному базису $\sigma_{3}, \sigma_{+}, \sigma_{-}$через $\beta, \alpha, \alpha^{\prime}$, а по ортонормированному $\vec{e}_{1}$, $\vec{e}_{2}, \vec{e}_{3}$ через $x, y, z$ (временно):

$$
\begin{gathered}
x=\frac{\alpha^{\prime}+\alpha}{i}, \quad y=\alpha^{\prime}-\alpha, \quad z=2 \frac{\beta}{i}, \\
\beta=i \frac{z}{2}, \quad \alpha=i \frac{x+i y}{2}, \quad \alpha^{\prime}=i \frac{x-i y}{2} .
\end{gathered}
$$

Пусть

$$
\begin{aligned}
& A=\beta_{1} \sigma_{3}+\alpha_{1} \sigma_{+}+\alpha_{1}^{\prime} \sigma_{-}=x_{1} \vec{e}_{1}+y_{1} \vec{e}_{2}+z_{1} \vec{e}_{3}, \\
& B=\beta_{2} \sigma_{3}+\alpha_{2} \sigma_{+}+\alpha_{2}^{\prime} \sigma_{-}=x_{2} \vec{e}_{1}+y_{2} \vec{e}_{2}+z_{2} \vec{e}_{3}, \\
& C=\beta_{3} \sigma_{3}+\alpha_{3} \sigma_{+}+\alpha_{3}^{\prime} \sigma_{-}=x_{3} \vec{e}_{1}+y_{3} \vec{e}_{2}+z_{3} \vec{e}_{3} .
\end{aligned}
$$

Тогда

$$
\begin{aligned}
\langle A,[B, C]\rangle_{\mathrm{e}} & =\operatorname{det}\left|\begin{array}{lll}
x_{1} & y_{1} & z_{1} \\
x_{2} & y_{2} & z_{2} \\
x_{3} & y_{3} & z_{3}
\end{array}\right| \\
& =\operatorname{det}\left|\begin{array}{lll}
\left(\alpha_{1}+\alpha_{1}^{\prime}\right) / i & \alpha_{1}^{\prime}-\alpha_{1} & 2 \beta_{1} / i \\
\left(\alpha_{2}+\alpha_{2}^{\prime}\right) / i & \alpha_{2}^{\prime}-\alpha_{2} & 2 \beta_{2} / i \\
\left(\alpha_{3}+\alpha_{3}^{\prime}\right) / i & \alpha_{3}^{\prime}-\alpha_{3} & 2 \beta_{3} / i
\end{array}\right|=-4 \operatorname{det}\left|\begin{array}{lll}
\beta_{1} & \alpha_{1} & \alpha_{1}^{\prime} \\
\beta_{2} & \alpha_{2} & \alpha_{2}^{\prime} \\
\beta_{3} & \alpha_{3} & \alpha_{3}^{\prime}
\end{array}\right|, \\
\langle A,[B, C]\rangle & =2 \operatorname{det}\left|\begin{array}{lll}
\beta_{1} & \alpha_{1} & \alpha_{1}^{\prime} \\
\beta_{2} & \alpha_{2} & \alpha_{2}^{\prime} \\
\beta_{3} & \alpha_{3} & \alpha_{3}^{\prime}
\end{array}\right| .
\end{aligned}
$$


5.2. Отображение $Q \sqrt{2 a_{i_{0} i_{0}}}$. Определим важнейшее для нас отображение набора $A^{(0)}, \ldots, A^{(n)}$ в проективное пространство. Основой будет следующее утверждение теории алгебр Ли [35], [36], не имеющее прямого аналога в вещественной евклидовой геометрии.

Tеорема 5. Для любого ненулевого $A^{(0)} \in \operatorname{sl}(2, \mathbb{C})$ величина

$$
\sqrt{2 a_{00}}:=\sqrt{2\left\langle A^{(0)}, A^{(0)}\right\rangle}=\sqrt{2 \operatorname{tr}\left(A^{(0)}\right)^{2}}=\sqrt{-4 \operatorname{det} A^{(0)}}
$$

является собственным числом линейного оператора $\operatorname{ad}_{A^{(0)}}$ :

$$
\operatorname{ad}_{A^{(0)}}=\left[A^{(0)}, \cdot\right]: \operatorname{sl}(2, \mathbb{C}) \rightarrow \operatorname{sl}(2, \mathbb{C}) .
$$

Соответствующее собственное подпространство одномерно, изотропно и ортогонально $A^{(0)}$.

ДокАЗАтельство. Воспользуемся тем, что любые два ненулевых элемента $A$ и $A^{\prime}$ из $\operatorname{sl}(2, \mathbb{C})$ с одинаковыми “квадратами длин" $\langle A, A\rangle=\left\langle A^{\prime}, A^{\prime}\right\rangle$, т. е. с одинаковыми собственными числами, можно совместить преобразованием из $\operatorname{SL}(2, \mathbb{C})$. Это - утверждение теоремы о приведении к жордановой форме для матриц из $\operatorname{sl}(2, \mathbb{C})$. Поскольку все утверждения $\mathrm{SL}(2, \mathbb{C})$-инвариантны, то достаточно рассмотреть два случая:

а) $a_{00} \neq 0, A^{(0)}=\sqrt{\frac{a_{00}}{2}}\left(\begin{array}{cc}1 & 0 \\ 0 & -1\end{array}\right)=\sqrt{\frac{a_{00}}{2}} \sigma_{3}$,

б) $a_{00}=0, A^{(0)}=\left(\begin{array}{ll}0 & 0 \\ 1 & 0\end{array}\right)=\sigma_{-}$.

Если $a_{00} \neq 0$, то у $\operatorname{ad}_{A^{(0)}}$ собственных подпространства два, они натянуты на изотропные собственные векторы $\sigma_{ \pm}$; соответствующие собственные числа равны $\pm \sqrt{2 a_{00}}$ :

$$
\left[\sqrt{\frac{a_{00}}{2}} \sigma_{3}, \sigma_{ \pm}\right]= \pm \sqrt{2 a_{00}} \sigma_{ \pm}
$$

Если $a_{00}=0$, то $\sigma_{-}-$единственный собственный вектор, соответствующий 0 , единственному собственному значению $\sqrt{2 a_{00}}=0$. Во всех случаях $\left\langle\sigma_{3}, \sigma_{ \pm}\right\rangle=$ $\left\langle\sigma_{-}, \sigma_{-}\right\rangle=0$, т. е. собственные векторы ортогональны $A^{(0)}$ и изотропны. Теорема доказана.

Пусть $\left(A^{(i)}, \widetilde{A}^{(i)}\right) \in \mathscr{C}_{a_{i i}}$ и $\sqrt{2 a_{i i}}-$ какое-нибудь значение корня.

ОБОзНАчЕНИЕ 3 . Обозначим через $\sigma^{\left(\sqrt{2 a_{i i}}\right)}$ какой-нибудь (любой, некоторый) собственный вектор оператора $\operatorname{ad}_{A^{(i)}}$, соответствующий собственному значению $\sqrt{2 a_{i i}}$, если $a_{i i} \neq 0$, и собственный вектор оператора $\operatorname{ad}_{\widetilde{A}^{(i)}}$, если $a_{i i}=0$.

Если $a_{i i}=0$ и $\widetilde{A}^{(i)}=\left(\begin{array}{cc}x_{3} & x_{1} \\ x_{2} & -x_{3}\end{array}\right), x_{1}: x_{2}: x_{3}=1:-p^{2}: p$, то можно выбрать

$$
\sigma^{\left(\sqrt{2 a_{i i}}\right)}=\left(\begin{array}{cc}
p & 1 \\
-p^{2} & -p
\end{array}\right)
$$


ОБОЗНАЧЕНИЕ 4 . Через $\left(\sigma^{\left(\sqrt{2 a_{i i}}\right)}\right)$ обозначим прямую с направляющим вектором $\sigma^{\left(\sqrt{2 a_{i i}}\right)}$.

Итак, для любого значения $\sqrt{2 a_{i i}} \in \mathbb{C}$ и любой точки $A^{(i)} \in \mathscr{C}_{a_{i i}}$ (вторую, проективную компоненту $\widetilde{A}^{(i)}$ пары $\left(A^{(i)}, \widetilde{A}^{(i)}\right) \in \mathscr{C}_{a_{i i}}$ здесь и далее мы не пишем - подразумеваем) однозначно определена изотропная прямая $\left(\sigma^{\left(\sqrt{2 a_{i i}}\right)}\right)$; через $\sigma^{\left(\sqrt{2 a_{i i}}\right)}$ обозначен какой-нибудь ее ненулевой (направляющий) вектор. Рассмотрим $\mathbb{C P}^{n}$ с однородными координатами $\alpha_{0}: \alpha_{1}: \cdots: \alpha_{n} \in \mathbb{C P}^{n}$. Обозначим $L_{Q}^{i} \subset \mathbb{C P}^{n}$ пересечение гиперплоскостей $\sum_{k=0}^{n} \alpha_{k}=0$ и $\alpha_{i}=0$. Очевидно, что $L_{Q}^{i} \sim \mathbb{C P}^{n-2}$ - это $(n-2)$-мерное проективное пространство.

ОБозНАчЕНиЕ 5 . Обозначим $\widetilde{Q}_{\sqrt{2 a_{j j}}}: \widetilde{M}_{a_{\bar{i} i}} \rightarrow L_{Q}^{j}$ следующее отображение:

$$
\left(A^{(0)}, \ldots, A^{(n)}\right) \mapsto\left\langle A^{(0)}, \sigma^{\left(\sqrt{2 a_{j j}}\right)}\right\rangle:\left\langle A^{(1)}, \sigma^{\left(\sqrt{2 a_{j j}}\right)}\right\rangle: \cdots:\left\langle A^{(n)}, \sigma^{\left(\sqrt{2 a_{j j}}\right)}\right\rangle,
$$

т. е. положим $\alpha_{k}:=\left\langle A^{(k)}, \sigma^{\left(\sqrt{2 a_{j j}}\right)}\right\rangle$.

УТВЕРЖДЕНИЕ 9. Определение $\widetilde{Q} \sqrt{2 a_{j j}}$ корректно.

ДокАЗАТЕЛЬСтво. Во-первых, отметим, что в определении точки раздутых дивизоров могут рассматриваться вместе с обыкновенными, ненулевыми $A^{(i)}$ - направление $\left(\sigma^{\left(\sqrt{2 a_{j j}}\right)}\right)$ определено корректно и в качестве $\sigma^{\left(\sqrt{2 a_{j j}}\right)}$ можно взять любой ненулевой вектор с этого направления. Если же какая-нибудь вершина $A^{(i)}$ с раздутого дивизора, то, конечно же, $\left\langle A^{(i)}, \sigma^{\left(\sqrt{2 a_{j j}}\right)}\right\rangle=0$.

Во-вторых, среди $\left\langle A^{(i)}, \sigma^{\left(\sqrt{2 a_{j j}}\right)}\right\rangle, i \in\{0, \ldots, n\}$, обязательно есть ненулевые, так как мы исключили из рассмотрения наборы $A^{(i)}$, состоящие из матриц, которые все могут быть приведены к одновременно-треугольному виду, иначе - ортогональные какому-нибудь одному изотропному вектору, в данном случае - вектору $\sigma^{\left(\sqrt{2 a_{j j}}\right)}$ (по теореме 5 вектор $\sigma^{\left(\sqrt{2 a_{j j}}\right)}$ изотропен). Таким образом, отображение $\widetilde{Q} \sqrt{2 a_{j j}}$, как отображение в $\mathbb{C} \mathbf{P}^{n}$, определено корректно.

Покажем, что образ $\widetilde{Q} \sqrt{2 a_{j j}}$ содержится в $L_{Q}^{j}$. Действительно, поскольку $\sum A^{(i)}=0$, то и $\sum_{i}\left\langle A^{(i)}, \sigma^{\left(\sqrt{2 a_{j j}}\right)}\right\rangle=0$; по теореме $5\left\langle A^{(j)}, \sigma^{\left(\sqrt{2 a_{j j}}\right)}\right\rangle=0$.

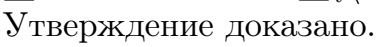

Отображение $\widetilde{Q}_{\sqrt{2 a_{j j}}}: \widetilde{M}_{a_{\bar{i}}} \rightarrow L_{Q}^{j}$ инвариантно относительно действия группы $\operatorname{SL}(2, \mathbb{C})$ на $\widetilde{M}_{a_{\bar{i}}}$, так что $\widetilde{Q} \sqrt{2 a_{j j}}$ может быть опущено на $M_{a_{\bar{i}}}$, навстречу направлению, задаваемому $\tilde{\pi}: \widetilde{M}_{a_{\bar{i} \bar{i}}} \rightarrow M_{a_{\bar{i} \bar{i}}}$.

Это отображение мы и обозначим $Q \sqrt{2 a_{j j}}: M_{a_{\bar{i}}} \rightarrow L_{Q}^{j}$.

5.3. Базис, сопутствующий $A^{(i)}, A^{(j)}, A^{(k)}$; условия его существования. Рассмотрим три вектора $A^{(0)}, A^{(n-1)}, A^{(n)}$ и зафиксируем какие-нибудь значения $\sqrt{2 a_{00}}, \sqrt{2 a_{n-1 n-1}}$.

Стандартный базис мы называем сопутствующим $A^{(0)}, A^{(n-1)}, A^{(n)}$ вдоль $\left(\sigma^{\left(-\sqrt{2 a_{00}}\right)}\right)$ и $\left(\sigma^{\left(\sqrt{2 a_{n-1 n-1}}\right)}\right)$, если

$$
\sigma_{-} \in\left(\sigma^{\left(-\sqrt{2 a_{00}}\right)}\right), \quad \sigma_{+} \in\left(\sigma^{\left(\sqrt{2 a_{n-1 n-1}}\right)}\right), \quad\left\langle\sigma_{-}, A^{(n)}\right\rangle=1 .
$$


УтВеРЖДЕНИЕ 10. Стандартный базис, сопутствующий $A^{(0)}, A^{(n-1)}, A^{(n)}$ вдоль $\left(\sigma^{\left(-\sqrt{2 a_{00}}\right)}\right)$ и $\left(\sigma^{\left(\sqrt{2 a_{n-1 n-1}}\right)}\right)$, существует и единствен тогда и только тогда, когда
a) $\left\langle\sigma^{\left(-\sqrt{2 a_{00}}\right)}, \sigma^{\left(\sqrt{2 a_{n-1} n-1}\right)}\right\rangle \neq 0$,
б) $\left\langle\sigma^{\left(-\sqrt{2 a_{00}}\right)}, A^{(n)}\right\rangle \neq 0$.

ДокАзАТЕЛЬство. Если условия а) и б) выполнены, то искомый базис (очевидно, единственный) дается явными формулами

$$
\begin{aligned}
\sigma_{-} & =\sigma^{\left(-\sqrt{2 a_{00}}\right)} \frac{1}{\left\langle\sigma^{\left(-\sqrt{2 a_{00}}\right)}, A^{(n)}\right\rangle}, \\
\sigma_{+} & =\sigma^{\left(\sqrt{2 a_{n-1 n-1}}\right)} \frac{\left\langle\sigma^{\left(-\sqrt{2 a_{00}}\right)}, A^{(n)}\right\rangle}{\left\langle\sigma^{\left(-\sqrt{2 a_{00}}\right)}, \sigma^{\left(\sqrt{2 a_{n-1 n-1}}\right)},\right.}, \\
\sigma_{3} & =\left[\sigma^{\left(\sqrt{2 a_{n-1 n-1}}\right)}, \sigma^{\left(-\sqrt{2 a_{00}}\right)}\right] \frac{1}{\left\langle\sigma^{\left(-\sqrt{2 a_{00}}\right)}, \sigma^{\left(\sqrt{2 a_{n-1 n-1}}\right)}\right\rangle} .
\end{aligned}
$$

Если условие а) не выполнено, то $\sigma_{-}$и $\sigma_{+}$линейно зависимы и $\sigma_{ \pm, 3}-$ не базис; нарушение условия б), т. е. равенство $\left\langle\sigma^{\left(-\sqrt{2 a_{00}}\right)}, A^{(n)}\right\rangle=0$, противоречит (50). Утверждение доказано.

Обозначим часть $\widetilde{M}_{a_{\bar{i}}}$, где выполнено $(51)$, через $\widetilde{\mathscr{U}}\left(-\sqrt{2 a_{00}} ; \sqrt{2 a_{n-1 n-1}}, n\right)$; иногда для краткости будем писать $\widetilde{\mathscr{U}}(\ldots)$ :

$$
\widetilde{\mathscr{U}}(\ldots):=\left\{A^{(\bar{i})} \in \widetilde{M}_{a_{\bar{i} \bar{i}}}:\left\langle\sigma^{\left(-\sqrt{2 a_{00}}\right)}, \sigma^{\left(\sqrt{2 a_{n-1}-1}\right)}\right\rangle \neq 0,\left\langle\sigma^{\left(-\sqrt{2 a_{00}}\right)}, A^{(n)}\right\rangle \neq 0\right\} .
$$

ТЕОРЕма 6. Для любого заданного набора значений $\sqrt{2 a_{i i}}, i \in\{0,1, \ldots, n\}$,

$$
\widetilde{M}_{a_{\overline{i i}}}=\bigcup_{\substack{i, j \\ 0 \neq i \neq j \neq 0}} \tilde{\mathscr{U}}\left(-\sqrt{2 a_{00}} ; \sqrt{2 a_{i i}}, j\right) .
$$

ДокАЗАТЕльство. Совпадение направлений $\left(\sigma^{\left(\sqrt{2 a_{i i}}\right)}\right)$ и $\left(\sigma^{\left(\sqrt{2 a_{j j}}\right)}\right)$ означает не только возможность приведения $A^{(j)}$ и $A^{(i)}$ к одинаково-треугольному (например верхнетреугольному) виду, но и некую согласованность, после такого приведения, аргументов (знаков) соответствующих диагональных элементов. Если же $A^{(j)}$ и $A^{(i)}$ просто верхнетреугольны, $A_{11}^{(i)}=\sqrt{a_{i i} / 2}$, аргумент $A_{11}^{(j)}-$ любой, то $\sigma^{\left(\sqrt{2 a_{i i}}\right)}=\sigma_{+}$и, конечно же, $\left\langle A^{(j)}, \sigma_{+}\right\rangle=A_{21}^{(j)}=0$. Следовательно,

$$
\left(\sigma^{\left(\sqrt{2 a_{i i}}\right)}\right)=\left(\sigma^{\left(\sqrt{2 a_{j j}}\right)}\right) \quad \Rightarrow \quad\left\langle A^{(j)}, \sigma^{\left(\sqrt{2 a_{i i}}\right)}\right\rangle=0
$$

Мы знаем, что все величины $\alpha_{i}:=\left\langle A^{(i)}, \sigma^{\left(\sqrt{2 a_{00}}\right)}\right\rangle$ равны нулю быть не могут, а так как $\sum \alpha_{i}=0$, то отличны от нуля минимум две из них.

Из этого следует, что для любого набора $A^{(\bar{i})}$ найдутся вершины $A^{(i)}$ и $A^{(j)}$ такие, что

$$
\left\langle A^{(i)}, \sigma^{\left(\sqrt{2 a_{00}}\right)}\right\rangle \neq 0 \neq\left\langle A^{(j)}, \sigma^{\left(\sqrt{2 a_{00}}\right)}\right\rangle
$$

и, значит, $A^{(\bar{i})}$ содержится в $\widetilde{U}\left(\sqrt{2 a_{00}} ; \sqrt{2 a_{i i}}, j\right)$. 
Формулы (52) задают отображение $A^{(\bar{k})} \rightarrow \sigma_{ \pm, 3}\left(A^{(\bar{k})}\right)$, дающее базис, подпадающий под действие утверждения 8. Отображение это определено на

$$
\widetilde{\mathscr{U}}\left(-\sqrt{2 a_{00}} ; \sqrt{2 a_{n-1 n-1}}, n\right) \subset \widetilde{M}_{a_{\overline{i i}}} .
$$

В соответствующем стандартном базисе матрицы имеют вид

$$
\begin{aligned}
A^{(0)} & =\left(\begin{array}{cc}
\sqrt{a_{00} / 2} & 0 \\
q_{0}^{\prime} & -\sqrt{a_{00} / 2}
\end{array}\right), & A^{(i)}=\left(\begin{array}{cc}
\beta_{i} & q_{i} \\
q_{i}^{\prime} & -\beta_{i}
\end{array}\right), \\
A^{(n-1)} & =\left(\begin{array}{cc}
\sqrt{a_{n-1 n-1} / 2} & q_{n-1} \\
0 & -\sqrt{a_{n-1 n-1} / 2}
\end{array}\right), & A^{(n)}=\left(\begin{array}{cc}
\beta_{n} & 1 \\
q_{n}^{\prime} & -\beta_{n}
\end{array}\right) .
\end{aligned}
$$

На эти формулы можно смотреть как на вложение $\widetilde{\mathscr{U}}(\ldots) / \mathrm{SL}(2, \mathbb{C}) \subset M_{a_{\overline{i i}}}$ в $\widetilde{M}_{a_{\bar{i}}}$. Взяв набор $A^{(\bar{i})}$ с $\widetilde{M}_{a_{\bar{i}}}$ и пользуясь формулами (52), мы получим базис $\sigma_{ \pm}, \sigma_{3}$. Разложение по этому базису (формулы $(24),(53)$ ) даст некоторую точку из $\widetilde{M}_{a_{\bar{i}}}$. Поскольку результат будет одинаковым для всех наборов с одной орбиты (построение сопутствующего базиса $\operatorname{SL}(2, \mathbb{C})$-инвариантно), то это отображение $\widetilde{\mathscr{U}}(\ldots) \rightarrow \widetilde{M}_{a_{\bar{i}}}$ можно рассматривать и как отображение $\widetilde{\mathscr{U}}(\ldots) / \mathrm{SL}(2, \mathbb{C}) \rightarrow \widetilde{M}_{a_{\bar{i}}}$. Это именно вложение, т. е. инъекция, потому что одинаковые матричные элементы, пусть даже в разных базисах, имеют наборы только связанные преобразованием из $\mathrm{SL}(2, \mathbb{C})$, с одной орбиты.

Уберем $A^{(0)}, A^{(n-1)}, A^{(n)}$ из набора $A^{(\bar{i})}$, т. е. спроектируем на произведение $n-2$ квадрик $\mathscr{C}_{a_{i i}}$ :

$$
\widetilde{\widetilde{M}}_{a_{\bar{i} i} \backslash\{0 n-1 n\}}:=\mathscr{C}_{a_{11}} \times \cdots \times \mathscr{C}_{a_{n-2 n-2}} .
$$

Эта проекция инъективна. Используя равенства

$$
\sum A^{(i)}=0 \quad \text { и } \quad \operatorname{det} A^{(n)}=-\frac{a_{n n}}{2},
$$

для каждой из отброшенных координат получаем формулу (отброшенные члены восстанавливаются):

$$
\begin{gathered}
-q_{n-1}=q_{\Sigma}+1, \quad q_{n-1}^{\prime}:=0, \quad \beta_{n-1}:=\sqrt{\frac{a_{n-1 n-1}}{2}}, \\
-\beta_{n}=\sqrt{\frac{a_{00}}{2}}+\sqrt{\frac{a_{n-1 n-1}}{2}}+\beta_{\Sigma} \\
q_{n}^{\prime}=-\left(\beta_{\Sigma}+\sqrt{\frac{a_{00}}{2}}+\sqrt{\frac{a_{n-1 n-1}}{2}}\right)^{2}+\frac{a_{n n}}{2} \\
-q_{0}^{\prime}=q_{\Sigma}^{\prime}-\left(\beta_{\Sigma}+\sqrt{\frac{a_{00}}{2}}+\sqrt{\frac{a_{n-1 n-1}}{2}}\right)^{2}+\frac{a_{n n}}{2} \\
q_{0}:=0, \quad \beta_{0}:=\sqrt{\frac{a_{00}}{2}}
\end{gathered}
$$

здесь

$$
\beta_{\Sigma}:=\sum_{i=1}^{n-2} \beta_{i}, \quad q_{\Sigma}:=\sum_{i=1}^{n-2} q_{i}, \quad q_{\Sigma}^{\prime}:=\sum_{i=1}^{n-2} q_{i}^{\prime} .
$$


Обозначим композицию вложения (53) и удаления матриц $A^{(0)}, A^{(n-1)}, A^{(n)}$ через $\pi_{\{0 n-1 n\}}$ :

$$
\pi_{\{0 n-1 n\}}: \widetilde{\mathscr{U}}\left(-\sqrt{2 a_{00}} ; \sqrt{2 a_{n-1 n-1}}, n\right) / \mathrm{SL}(2, \mathbb{C}) \rightarrow \widetilde{\widetilde{M}}_{a_{\bar{i} \bar{i}} \backslash\{0 n-1 n\}} .
$$

Нами доказано следующее утверждение.

УТВЕРЖДЕНИЕ 11. Отображение $\pi_{\{0 n-1 n\}}$ является биекиией.

Вернемся к иллюстративному примеру с поверхностью вращения (с. 55). Аналог пространства $\widetilde{M}_{a_{\bar{i}}}$ - это $X Y$-плоскость, аналог пространства $M_{a_{\bar{i}}}=$ $\widetilde{M}_{a_{\bar{i}}} / \mathrm{SL}(2, \mathbb{C})$ одномерен, точками этого многообразия являются концентрические окружности.

Отображение $\widetilde{\mathscr{U}}(\ldots) \rightarrow \widetilde{M}_{a_{\bar{i}}}$, состоящее в построении сопутствующего базиса и последующего разложения по нему набора $A^{(\bar{i})}$ (формулы (53)), аналогично отображению части плоскости $X Y$ (например кругового кольца) в какую-нибудь параметризующую кривую, один раз пересекающую все окружности этого кольца. Если отождествить окружность с той точкой параметризующей кривой, в которой они (окружность и кривая) пересекаются, получится вложение $\widetilde{M}_{a_{\overline{i i}}} / \mathrm{SL}(2, \mathbb{C})$ в плоскость $X Y$, образом которого является эта параметризующая кривая.

Развивая аналогию, укажем, что традиционная параметризация с помощью нормировки $\Psi\left(\infty_{\lambda}\right)=$ const (проекция решения системы Шлезингера) может быть уподоблена выбору в качестве параметризующей кривой какого-нибудь луча, выходящего из начала координат. Система (53) соответствует некоторой более экзотической кривой. Такими кривыми все множество окружностей покроется не сразу (как лучом), а несколькими частями. Смысл этого на примере не виден. Состоит он в том, что параметризующая кривая не самощель, она должна быть далее сама параметризована симплектическими $(p, q)$-картами ( $q$ - это искомая функция в уравнении Пенлеве VI). Луч покрывается такими картами плохо, так как $(p, q)$-карты для него слишком маленькие, а предложенная кривая (53) хорошо - ее накрывает всего пара карт, связанных инволютивным преобразованием $(p, q) \rightleftarrows\left(1 / p,-p\left(p q+\sqrt{2 a_{00}}\right)\right)$.

\section{4. Симплектичность пространства $M_{a_{\bar{i}}}$, построение сопутствую-} щего базиса по данному набору $A^{(\bar{k})}$. Многообразие $\widetilde{\widetilde{M}}_{a_{\bar{i} \bar{i}} \backslash\{0 n-1 n\}}$ симплектическое - как произведение симплектических многообразий, обозначим его 2-форму $\widetilde{\widetilde{\omega}}_{\{0 n-1 n\}}$.

ТЕОРЕма 7. Форма $\left.\widetilde{\widetilde{\omega}}\right|_{\widetilde{M} \subset \widetilde{\widetilde{M}}}$, являющаяся сужением симплектической формы $\widetilde{\widetilde{\omega}}$ на $\widetilde{\mathscr{U}}\left(-\sqrt{2 a_{00}} ; \sqrt{2 a_{n-1 n-1}}, n\right) \subset \widetilde{M}_{a_{\overline{i i}}} \subset \widetilde{\widetilde{M}}_{a_{\overline{i i}}}$, совпадает с поднятием $\widetilde{\widetilde{\omega}}_{\{0 n-1 n\}}$ на $\tilde{\mathscr{U}}(\ldots)$ :

$$
\tilde{\pi}^{*} \circ \pi_{\{0 n-1 n\}}^{*} \widetilde{\widetilde{\omega}}_{\{0 n-1 n\}}=\left.\widetilde{\widetilde{\omega}}\right|_{\tilde{M} \subset \widetilde{\widetilde{M}}} .
$$


ДокАзАтельство. Сужение $\widetilde{\widetilde{\omega}}$ на $\widetilde{M}_{a_{\overline{i i}}}$ не зависит от выбора базиса $\operatorname{sl}(2, \mathbb{C})$ (утверждение 2), так что мы можем вычислять сумму $\sum_{i=0}^{n} \omega^{(i)}$ в сопутствующем вдоль $\left(\sigma^{\left(-\sqrt{2 a_{00}}\right)}\right)$ и $\left(\sigma^{\left(\sqrt{2 a_{n-1 n-1}}\right)}\right)$ базисе, который существует для $A^{(\bar{i})} \in$ $\widetilde{\mathscr{U}}\left(-\sqrt{2 a_{00}} ; \sqrt{2 a_{n-1 n-1}}, n\right)$.

В таком базисе $\omega^{(0)}=\omega^{(n-1)}=\omega^{(n)}=0$ ! Это следствие того, что $A^{(0)}$, $A^{(n-1)}, A^{(n)}$ лежат на одномерных подмногообразиях - на пересечениях квадрик $\mathscr{O}_{a_{i i}}(i=0, n-1, n)$ и плоскостей $A_{12}^{(0)}=0, A_{21}^{(n-1)}=0, A_{12}^{(n)}=1$, так что

$$
\sum_{i=0}^{n} \omega^{(i)}=\sum_{i=1}^{n-2} \omega^{(i)}
$$

Обозначим

$$
\mathscr{U}(\ldots):=\tilde{\pi}(\tilde{\mathscr{U}}(\ldots)) \subset M_{a_{\bar{i} i}} .
$$

Определение $\widetilde{\mathscr{U}}(\ldots)$ является $\operatorname{SL}(2, \mathbb{C})$-инвариантным, так что

$$
\widetilde{\mathscr{U}}(\ldots)=\tilde{\pi}^{-1}(\mathscr{U}(\ldots))
$$

и, значит,

$$
M_{a_{\bar{i}}}=\bigcup_{\substack{i, j \\ 0 \neq i \neq j \neq 0}} \mathscr{U}\left(-\sqrt{2 a_{00}} ; \sqrt{2 a_{i i}}, j\right) .
$$

Многообразие $\mathscr{U}\left(-\sqrt{2 a_{i i}} ; \sqrt{2 a_{j j}}, k\right)$ - симплектическое пространство, оно изоморфно $\widetilde{\widetilde{M}}_{a_{\bar{i} i} \backslash\{i j k\}}$ - декартову произведению $n-2$ квадрик $\prod_{m \notin\{i, j, k\}} \mathscr{C}_{a_{m m}}$, причем мы доказали, что изоморфизм $\pi_{\{i j k\}}$ симплектический и бирациональный. Таким образом, верна теорема (более абстрактное доказательство симплектичности $M_{a_{\bar{i}}}$ см. в [7], [37]).

Теорема 8. Многообразие $M_{a_{\bar{i}}}$ является симплектическим. Симплектическая форма $\omega$ на нем склеивается из форм на $\mathscr{U}\left(-\sqrt{2 a_{00}} ; \sqrt{2 a_{i i}}, j\right) \simeq$ $\widetilde{\widetilde{M}}_{a_{\overline{i i}} \backslash\{0 i j\}}$, совпадающих на перекрытиях (почти везде).

ЗАмечАниЕ 21. Набором локальных канонических координат на $M_{a_{\bar{i}}}$ является любой набор $\left(p_{i}, q_{i}\right)_{i=1}^{n-2}$, где $\left(p_{i}, q_{i}\right)$ - произвольная пара (локальных) симплектических координат на квадрике $\mathscr{O}_{a_{i i}}$.

Теорема 9. Координатные функции $a_{i k}, f_{i j k}$ и функции $\beta_{i}, q_{i}, q_{i}^{\prime}$ - матричные элементы $A^{(i)}$ в сопутствующем базисе - связаны бирационально.

ДокАЗАТЕЛьСтво. В одну сторону это очевидно. Используя представление (53) и формулы (54), можно вычислить $\operatorname{tr} A^{(i)} A^{(j)}=a_{i j}$ и $\operatorname{tr}\left[A^{(i)}, A^{(j)}\right] A^{(k)}=$ $f_{i j k}$, они будут рациональными функциями (многочленами) от матричных элементов $\beta_{i}, q_{i}, q_{i}^{\prime}, i=1, \ldots, n-2$.

Пусть теперь заданы $a_{i k}, f_{i j k}$. Обозначим

$$
\sigma^{(\sqrt{2\langle A, A\rangle})}(B \backslash A):=\langle A, A\rangle B-\langle A, B\rangle A+\sqrt{\langle A, A\rangle / 2}[A, B] .
$$

Непосредственной подстановкой (используются формулы (45), (46)) легко проверяется следующее утверждение. 
УтверЖДЕНИЕ 12. Для любых $A, B \in \operatorname{sl}(2, \mathbb{C})$ вектор $\sigma=\sigma^{(\sqrt{2\langle A, A\rangle})}(B \backslash A)$ удовлетворяет равенству $[A, \sigma]=\sqrt{2\langle A, A\rangle} \sigma$.

Из условия одновременной нетреугольности следует, что среди $A^{(\bar{i})}$ всегда найдутся $A^{\widehat{0}}$ и $A^{n-1}$ такие, что

$$
\sigma^{\left(-\sqrt{2 a_{00}}\right)}\left(A^{(\widehat{0})} \backslash A^{(0)}\right) \neq 0, \quad \sigma^{\left(\sqrt{2 a_{n-1}-1}\right)}\left(A^{(\widehat{n-1})} \backslash A^{(n-1)}\right) \neq 0 .
$$

Положим

$$
\begin{aligned}
\sigma^{\left(-\sqrt{2 a_{00}}\right)} & =\sigma^{\left(-\sqrt{2 a_{00}}\right)}\left(A^{(\widehat{0})} \backslash A^{(0)}\right), \\
\sigma^{\left(\sqrt{2 a_{n-1}-1}\right)}: & =\sigma^{\left(\sqrt{2 a_{n-1}-1}\right)}\left(A^{(\widehat{n-1})} \backslash A^{(n-1)}\right) .
\end{aligned}
$$

Для $A^{(\bar{i})} \in \widetilde{\mathscr{U}}\left(-\sqrt{2 a_{00}} ; \sqrt{2 a_{n-1}-1}, n\right)$ векторы $\sigma^{\left(-\sqrt{2 a_{00}}\right)}$ и $\sigma^{\left(\sqrt{2 a_{n-1}-1}\right)}$ линейно независимы и формулы (52) для (56) дадут стандартный базис $\sigma_{ \pm, 3}$, сопутствующий $A^{(\bar{i})}$. Матричные элементы $\beta_{i}, q_{i}, q_{i}^{\prime}-$ это $\left\langle\sigma_{3} / 2, A^{(i)}\right\rangle,\left\langle\sigma_{-}, A^{(i)}\right\rangle$, $\left\langle\sigma_{+}, A^{(i)}\right\rangle$; мы получили их рациональное представление через $a_{i j}$ и $f_{i j k}$.

Закончим этот раздел доказательством одной красивой геометрической формулы. Пусть $n=3$, т. е. имеются четыре вектора $A^{(0)}, A^{(1)}, A^{(2)}, A^{(3)}$ постоянной длины и суммой нуль, т. е. (вообще говоря) не плоский ориентированный четырехугольник в пространстве, с зафиксированными "длинами сторон" (их квадратами) $a_{i i}:=\left\langle A^{(i)}, A^{(i)}\right\rangle=$ const. Построим стандартный базис $\sigma_{-}$, $\sigma_{+}, \sigma_{3}$, сопутствующий векторам $A^{(0)}, A^{(2)}, A^{(3)}$ вдоль направлений $\left(\sigma^{\left(-\sqrt{2 a_{00}}\right)}\right)$ и $\left(\sigma^{\left(\sqrt{2 a_{22}}\right)}\right)$, т. е. в этом базисе

$$
A_{11}^{(0)}=+\sqrt{\frac{a_{00}}{2}}, \quad A_{11}^{(2)}=+\sqrt{\frac{a_{22}}{2}}, \quad A_{12}^{(3)}=1
$$

(при выводе уравнения Пенлеве VI мы будем использовать именно такую нормировку, т. е. знак при $\left.\sqrt{a_{00} / 2}\right)$. Вычислим ориентированный объем параллелепипеда, натянутого на $A^{(1)}, A^{(2)}, A^{(0)}$ :

$$
\begin{aligned}
f_{120} & =\left\langle A^{(1)},\left[A^{(2)}, A^{(0)}\right]\right\rangle=2 \operatorname{det}\left|\begin{array}{ccc}
\beta_{1} & q_{1} & q_{1}^{\prime} \\
\sqrt{a_{22} / 2} & q_{2} & 0 \\
\sqrt{a_{00} / 2} & 0 & q_{0}^{\prime}
\end{array}\right| \\
& =2 q_{0}^{\prime}\left(\beta_{1} q_{2}-\sqrt{\frac{a_{22}}{2}} q_{1}\right)-\sqrt{2 a_{00}} q_{2} q_{1}^{\prime} .
\end{aligned}
$$

Так как $M_{a_{\bar{i}}}$ при $n=3$ двумерно, имеется ровно одна пара канонических переменных $\left(p_{1}, q_{1}\right)$, и форма $d a_{01} \wedge d a_{02}$ обязана быть пропорциональной $d p_{1} \wedge d q_{1}$ : $d a_{01} \wedge d a_{02}=V d p_{1} \wedge d q_{1}$, причем коэффициент пропорциональности $V$ не может иметь особенностей; вычислим его. 
Учтем, что

$$
\begin{gathered}
\beta_{0}=\sqrt{\frac{a_{00}}{2}}=\text { const, } \quad \beta_{2}=\sqrt{\frac{a_{22}}{2}}=\text { const, } \quad q_{0}=0, \quad q_{2}^{\prime}=0 ; \\
a_{0 i}=2 \sqrt{\frac{a_{00}}{2}} \beta_{i}+q_{0}^{\prime} q_{i}, \quad q_{0}^{\prime}=-q_{1}^{\prime}-q_{3}^{\prime}, \\
q_{2}=-q_{1}-1, \quad-q_{1}^{\prime}=p_{1}\left(\beta_{1}+\sqrt{2 a_{11}}\right), \\
-q_{3}^{\prime}=\left(\beta_{1}+\sqrt{\frac{a_{00}}{2}}+\sqrt{\frac{a_{22}}{2}}\right)^{2}-\frac{a_{33}}{2}, \quad \beta_{1}=p_{1} q_{1}+\sqrt{\frac{a_{11}}{2}}
\end{gathered}
$$

и подставим в $d a_{01} \wedge d a_{02}$ :

$$
\begin{aligned}
d a_{01} \wedge d a_{02} & =d\left(\sqrt{2 a_{00}} \beta_{1}+q_{0}^{\prime} q_{1}\right) \wedge d\left(q_{0}^{\prime} q_{2}\right) \\
& =\sqrt{2 a_{00}} d \beta_{1} \wedge d\left(q_{0}^{\prime} q_{2}\right)+d\left(q_{0}^{\prime} q_{1}\right) \wedge d\left(q_{0}^{\prime} q_{2}\right) \\
& =q_{0}^{\prime} d\left(q_{0}^{\prime}-\sqrt{2 a_{00}} \beta_{1}\right) \wedge d q_{1}+\sqrt{2 a_{00}} q_{2}\left(\beta_{1}+\sqrt{\frac{a_{11}}{2}}\right) d \beta_{1} \wedge d p_{1} .
\end{aligned}
$$

Мы также использовали, что $d q_{2}=-d q_{1}$ и $d \beta_{1} \wedge d q_{3}^{\prime}=0$, поэтому

$$
d \beta_{1} \wedge d q_{0}^{\prime}=-d \beta_{1} \wedge d q_{1}^{\prime}=\left(\beta_{1}+\sqrt{\frac{a_{11}}{2}}\right) d \beta_{1} \wedge d p_{1},
$$

так что

$$
\begin{aligned}
V= & \left.q_{0}^{\prime} \frac{\partial}{\partial p_{1}}\right|_{q_{1}}\left(q_{0}^{\prime}-\sqrt{2 a_{00}} \beta_{1}\right)-\left.\sqrt{2 a_{00}} q_{2}\left(\beta_{1}+\sqrt{\frac{a_{11}}{2}}\right) \frac{\partial}{\partial q_{1}}\right|_{p_{1}} \beta_{1} \\
= & q_{0}^{\prime}\left(2 p_{1} q_{1}+2 \sqrt{\frac{a_{11}}{2}}+\left(2\left(\beta_{1}+\sqrt{\frac{a_{00}}{2}}+\sqrt{\frac{a_{11}}{2}}\right)-\sqrt{2 a_{00}}\right) q_{1}\right) \\
& -\sqrt{2 a_{00}} q_{2}\left(\beta_{1}+\sqrt{\frac{a_{11}}{2}}\right) p_{1} \\
=2 & q_{0}^{\prime}\left(\beta_{1} q_{1}+p_{1} q_{1}+\sqrt{\frac{a_{11}}{2}}+\sqrt{\frac{a_{22}}{2}} q_{1}\right) \\
& +\sqrt{2 a_{00}} q_{2} q_{1}^{\prime}=-2 q_{0}^{\prime}\left(\beta_{1} q_{2}-\sqrt{\frac{a_{22}}{2}} q_{1}\right)+\sqrt{2 a_{00}} q_{2} q_{1}^{\prime}=-f_{120} .
\end{aligned}
$$

Нами доказано следующее утверждение.

УтвеРЖДЕНИЕ 13. Симплектическая форма $\omega$ имеет представление

$$
d p_{1} \wedge d q_{1}=\frac{d a_{01} \wedge d a_{02}}{f_{123}} .
$$

Отметим, что справа стоит выражение из элементарной геометрии - скалярные произведения сторон четырехугольника (это $a_{01}$ и $a_{02}$ ) и объем $f_{123}$ его выпуклой оболочки (четырехугольник не плоский).

Равенство (57) можно рассматривать как геометрическое определение симплектической формы на пространстве тетраэдров (о симплектической геометрии пространства многоугольников см. [38]). Удивительно, что с нашим определением $\omega$, как суммы заметаемых направлениями сторон телесных углов, форма $\left(d a_{01} \wedge d a_{02}\right) / f_{123}$ связана столь сложным вычислением. 


\section{6. Система Гарнье, уравнение Пенлеве VI}

Системой Гарнье [1] называется каноническая система уравнений изомонодромных деформаций (43), записанная в канонических переменных $p_{i}, q_{i}$, т. е.

$$
\begin{gathered}
\frac{\partial}{\partial \lambda_{i}} q_{j}=\frac{\partial}{\partial p_{j}} H_{i}, \quad \frac{\partial}{\partial \lambda_{i}} p_{j}=-\frac{\partial}{\partial q_{j}} H_{i}, \quad i, j \in\{1, \ldots, n-2\}, \\
H_{i}=\sum_{\substack{k=0 \\
k \neq i}}^{n} \frac{a_{i k}}{\lambda_{i}-\lambda_{k}}, \quad i=1, \ldots, n-2,
\end{gathered}
$$

где $a_{i j}=a_{i j}\left(p_{\bar{m}}, q_{\bar{m}}\right)$ - многочлены (теорема 9$) ; \lambda_{0}, \lambda_{n-1}, \lambda_{n}-$ различные константы, например, $\lambda_{0}=0, \lambda_{n-1}=1, \lambda_{n}=\infty$ (в этом случае члены суммы, содержащие $\lambda_{n}$, отсутствуют, суммирование по $k$ от 1 до $\left.n-1\right)$. Важнейший случай этой системы, случай Пенлеве VI, рассмотрим детально.

Шестое уравнение Пенлеве - это уравнение Эйлера-Лагранжа, соответствующее гамильтоновой системе (58) (простейший нетривиальный случай, $n=3$; этот подход развивается в [39], [40]), записанной в канонических переменных $p, q$ какого-нибудь из многообразий $\widetilde{\widetilde{M}}_{a_{\bar{i} i} \backslash\{i j k\}}$.

Если $n=3$, то $n-2=1$, т. е. имеется единственная пара канонических переменных, многообразие

$$
M_{a_{\bar{i} i}}=\bigcup_{i, j: i \neq 0 \neq j} \widetilde{\widetilde{M}}_{a_{\overline{i i}} \backslash\{0 i j\}}=\bigcup_{k=1}^{3} \widetilde{\widetilde{M}}_{a_{k k}}
$$

двумерно, расширенное фазовое пространство $M_{a_{\bar{i}}}^{\Lambda}$ трехмерно.

Чтобы выписать само уравнение, нужно переписать гамильтониан

$$
H=\sum a_{i j} \log \left(\lambda_{i}-\lambda_{j}\right)
$$

заданный в координатах $a_{i j}$, через канонические координаты $p, q$ и сделать по $p$ преобразование Лежандра - исключить $p$ из канонической системы уравнений. Ввиду важности уравнения $\mathrm{P}^{\mathrm{VI}}$ приведем соответствующие вычисления. Начиная с этого момента, $n=3$; набор $A^{(\bar{i})}=A^{(0)}, A^{(1)}, A^{(2)}, A^{(3)}$ будем называть тетраэдром, апеллируя к геометрической интуиции:

$$
\left(\mathbb{R}^{3}, \ldots \times \ldots\right) \sim(\operatorname{su}(2),[\cdot, \cdot]) \subset(\operatorname{sl}(2, \mathbb{C}),[\cdot, \cdot]) .
$$

Мы должны зафиксировать область $\mathscr{U}\left(\sqrt{2 a_{i i}} ; \sqrt{2 a_{j j}}, k\right)$, т. е. индексы $i, j, k$ и два значения корней из параметров.

Положим $i=0, j=2, k=3$; если фиксировать $\mathscr{U}\left(\sqrt{2 a_{00}} ; \sqrt{2 a_{22}}, 3\right)$, то в сопутствующем стандартном базисе будет

$$
A_{11}^{(0)}=-\sqrt{\frac{a_{00}}{2}}, \quad A_{11}^{(2)}=+\sqrt{\frac{a_{22}}{2}},
$$

но этот знак минус у $A_{11}^{(0)}$ нарушит (с точки зрения автора) симметрию возникающих формул, так что мы зафиксируем область $\mathscr{U}\left(-\sqrt{2 a_{00}} ; \sqrt{2 a_{22}}, 3\right)$ и будем 
строить стандартный базис $\left(\sigma_{-}, \sigma_{+}, \sigma_{3}\right)$, сопутствующий $A^{(0)}, A^{(2)}, A^{(3)}$ вдоль направлений ${ }^{4}\left(\sigma^{\left(-\sqrt{2 a_{00}}\right)}\right)$ и $\left(\sigma^{\left(+\sqrt{2 a_{22}}\right)}\right)$.

Стандартный сопутствующий базис $\sigma_{-}, \sigma_{+}, \sigma_{3}$ определен так, что векторы $A^{(0)}, A^{(2)}$ и $A^{(3)}$ движутся по линиям - сечениям квадрик $\mathscr{C}_{a_{i i}}$ плоскостями $\left\langle A^{(0)}, \sigma_{-}\right\rangle=0,\left\langle A^{(2)}, \sigma_{+}\right\rangle=0$ и $\left\langle A^{(3)}, \sigma_{-}\right\rangle=1$. Эти линии однозначно проектируются на соответствующие координатные оси, в результате каждая из матриц $A^{(0)}, A^{(2)}$ и $A^{(3)}$ определяется единственным своим матричным элементом:

$$
\begin{gathered}
A^{(0)}=\left(\begin{array}{cc}
\sqrt{a_{00} / 2} & 0 \\
q_{0}^{\prime} & -\sqrt{a_{00} / 2}
\end{array}\right), \quad A^{(2)}=\left(\begin{array}{cc}
\sqrt{a_{22} / 2} & q_{2} \\
0 & -\sqrt{a_{22} / 2}
\end{array}\right), \\
A^{(3)}=\left(\begin{array}{cc}
\beta_{3} & 1 \\
a_{33} / 2-\beta_{3}^{2} & -\beta_{3}
\end{array}\right) .
\end{gathered}
$$

Вследствие равенства $\sum A^{(i)}=0$, по вершине $A^{(1)}$ - точке квадрики $\mathscr{C}_{a_{11}}$ восстанавливается весь тетраэдр:

$$
\begin{aligned}
& \beta_{3}=-\left(\beta_{0}+\beta_{1}+\beta_{2}\right)=-\left(\sqrt{\frac{a_{00}}{2}}+\beta_{1}+\sqrt{\frac{a_{22}}{2}}\right) \\
& q_{2}=-\left(q_{0}+q_{1}+q_{3}\right)=-\left(q_{1}+1\right) \\
& q_{0}^{\prime}=-\left(q_{1}^{\prime}+q_{2}^{\prime}+q_{3}^{\prime}\right)=-\left(q_{1}^{\prime}+\frac{a_{33}}{2}-\left(\sqrt{\frac{a_{00}}{2}}+\sqrt{\frac{a_{22}}{2}}+\beta_{1}\right)^{2}\right) .
\end{aligned}
$$

Заметим, что для всех (разных) точек $A^{(1)}$ с раздутого дивизора (существующего, если $a_{11}=0$ ) получатся одни и те же $A^{(0)}, A^{(2)}, A^{(3)}$ - некоторый конкретный треугольник.

Обратно, для любого тетраэдра $A^{(\bar{i})}$ из $\widetilde{\mathscr{U}}\left(-\sqrt{2 a_{00}} ; \sqrt{2 a_{22}}, 3\right)$ можно построить стандартный сопутствующий базис. Формулы

$$
\beta_{1}=-\left(\sqrt{\frac{a_{00}}{2}}+\sqrt{\frac{a_{22}}{2}}+\beta_{3}\right), \quad q_{1}=-\left(1+q_{2}\right), \quad q_{1}^{\prime}=-\left(q_{0}^{\prime}+\frac{a_{33}}{2}-\beta_{3}^{2}\right)
$$

показывают, что по значениям координат $\beta_{3}, q_{2}, q_{0}^{\prime}$ точка $A^{(1)}$ восстанавливается однозначно.

Заметим, что так как $\beta_{1}^{2}+q_{1} q_{1}^{\prime}=a_{11} / 2$, то величины $\beta_{3}, q_{2}$ и $q_{0}^{\prime}$ не являются независимыми:

$$
\left(\sqrt{\frac{a_{00}}{2}}+\sqrt{\frac{a_{22}}{2}}+\beta_{3}\right)^{2}+\left(1+q_{2}\right)\left(q_{0}^{\prime}+\frac{a_{33}}{2}-\beta_{3}^{2}\right)=\frac{a_{11}}{2} .
$$

Скалярные произведения $a_{0 i}=\left\langle A^{(0)}, A^{(i)}\right\rangle$ (остальные из них - посредством формул (38)) выражаются через матричные элементы $\beta_{i}, q_{i}, q_{i}^{\prime}$ следующим

\footnotetext{
${ }^{4}$ Конечно же, во всех формулах можно поменять $\sqrt{a_{i i}} \rightarrow-\sqrt{a_{i i}}$ для произвольного (или некоторых) $i$, в частности $\sqrt{a_{00}} \rightarrow-\sqrt{a_{00}}$.
} 
образом (вычисляем след произведения матриц $A^{(0)}$ и $\left.A^{(i)}\right)$ :

$$
\begin{gathered}
a_{0 i}=2 \beta_{0} \beta_{i}+q_{0} q_{i}^{\prime}+q_{i} q_{0}^{\prime}=2 \beta_{0} \beta_{i}+q_{i} q_{0}^{\prime} \\
=\sqrt{2 a_{00}} \beta_{i}+q_{i}\left(q_{2} q_{1}^{\prime}+2 \beta_{1}\left(\sqrt{\frac{a_{00}}{2}}+\sqrt{\frac{a_{22}}{2}}\right)\right. \\
\left.+\frac{a_{00}}{2}+\frac{a_{22}}{2}+\frac{a_{11}}{2}-\frac{a_{33}}{2}+\sqrt{a_{00} a_{33}}\right) .
\end{gathered}
$$

Мы учли, что $q_{0}^{\prime}=-\left(q_{1}^{\prime}+q_{2}^{\prime}+q_{3}^{\prime}\right), q_{2}^{\prime}=0$ и $\beta_{1}^{2}=a_{11} / 2-q_{1} q_{1}^{\prime}$. Все матричные элементы - многочлены от координат $q_{1}$ и $p_{1}=\left(\beta_{1}-\sqrt{a_{11} / 2}\right) / q_{1}$ :

$$
\beta_{1}=p_{1} q_{1}+\sqrt{\frac{a_{11}}{2}}, \quad q_{1}^{\prime}=-p_{1}\left(p_{1} q_{1}+2 \sqrt{\frac{a_{11}}{2}}\right)=-p_{1}^{2} q_{1}-2 \sqrt{\frac{a_{11}}{2}} p_{1},
$$

так что

$$
\begin{aligned}
a_{0 i}=- & q_{i} q_{1} q_{2} p_{1}^{2}+2 p_{1} q_{i}\left(q_{1}\left(\sqrt{\frac{a_{00}}{2}}+\sqrt{\frac{a_{22}}{2}}\right)-q_{2} \sqrt{\frac{a_{11}}{2}}\right) \\
+ & q_{i}\left(\left(\sqrt{\frac{a_{00}}{2}}+\sqrt{\frac{a_{11}}{2}}+\sqrt{\frac{a_{22}}{2}}\right)^{2}-\frac{a_{33}}{2}\right)+\beta_{i} \sqrt{2 a_{00}}
\end{aligned}
$$

Подставляя $\beta_{1}=p_{1} q_{1}+\sqrt{a_{11} / 2}, \beta_{2}=\sqrt{a_{22} / 2}, \beta_{3}=-\beta_{1}-\left(\sqrt{a_{00} / 2}+\sqrt{a_{22} / 2}\right)$, $\beta_{0}=\sqrt{a_{00} / 2}$, получаем

$$
\begin{aligned}
a_{01}=- & q_{1} q_{1} q_{2} p_{1}^{2}+2 q_{1} p_{1}\left(q_{1} \sqrt{\frac{a_{22}}{2}}-q_{2}\left(\sqrt{\frac{a_{11}}{2}}+\sqrt{\frac{a_{00}}{2}}\right)\right) \\
& +q_{1}\left(\left(\sqrt{\frac{a_{00}}{2}}+\sqrt{\frac{a_{11}}{2}}+\sqrt{\frac{a_{22}}{2}}\right)^{2}-\frac{a_{33}}{2}\right)+\sqrt{a_{00} a_{11}} \\
a_{02}=- & q_{2} q_{1} q_{2} p_{1}^{2}+2 q_{2} p_{1}\left(q_{1}\left(\sqrt{\frac{a_{00}}{2}}+\sqrt{\frac{a_{22}}{2}}\right)-q_{2} \sqrt{\frac{a_{11}}{2}}\right) \\
& +q_{2}\left(\left(\sqrt{\frac{a_{00}}{2}}+\sqrt{\frac{a_{11}}{2}}+\sqrt{\frac{a_{22}}{2}}\right)^{2}-\frac{a_{33}}{2}\right)+\sqrt{a_{00} a_{22}}, \\
a_{03}=- & q_{3} q_{1} q_{2} p_{1}^{2}+2 q_{3} p_{1}\left(q_{1} \sqrt{\frac{a_{22}}{2}}-q_{2} \sqrt{\frac{a_{11}}{2}}\right) \\
& +q_{3}\left(\left(\sqrt{\frac{a_{00}}{2}}+\sqrt{\frac{a_{11}}{2}}+\sqrt{\frac{a_{22}}{2}}\right)^{2}-\frac{a_{33}}{2}\right) \\
& -\sqrt{a_{00}}\left(\sqrt{a_{00}}+\sqrt{a_{11}}+\sqrt{a_{22}}\right) .
\end{aligned}
$$

Здесь $q_{3}:=1, \sqrt{a_{i i} a_{j j}}:=\sqrt{a_{i i}} \sqrt{a_{j j}}$.

Рассмотрим линейную комбинацию $\sum a_{0 i} t_{i}$, где $t_{1}, t_{2}, t_{3}$ - какие-то комплексные параметры. Сразу видно, что сумма $\sum a_{0 i} t_{i}$ будет иметь вид

$$
p_{1}^{2} \mathscr{P}_{3}+2 p_{1} \mathscr{P}_{2}+\mathscr{P}_{1}
$$

где $\mathscr{P}_{k}-$ многочлен степени не выше " $k$ " от переменной $q_{1}$. 
Если $t_{1} \neq t_{2}$, то $\mathscr{P}_{3}$ действительно имеет третью степень по $q_{1}$; если же $t_{1}=t_{2}$, то $\sum a_{0 i} t_{i}$ линейно по $q_{1}$ :

$\left(t_{1}-t_{3}\right)\left(q_{1} q_{2} p_{1}^{2}-\left(\sqrt{\frac{a_{00}}{2}}+\sqrt{\frac{a_{11}}{2}}+\sqrt{\frac{a_{22}}{2}}\right)^{2}-\frac{a_{33}}{2}+\sqrt{a_{00}}\left(\sqrt{a_{11}}+\sqrt{a_{22}}\right)\right)-a_{00} t_{3}$.

Поскольку все скалярные произведения $\left\langle A^{(i)}, A^{(j)}\right\rangle$ выражаются линейно через $a_{0 k}$ и константы (формула $(38)$ ), то верна теорема, используемая К. Окамото и, по-видимому, известная еще классикам - Фуксу и Пенлеве.

Tеорема 10. Пусть $A^{\prime}:=\sum \nu_{i}^{\prime} A^{(i)}$ u $A^{\prime \prime}:=\sum \nu_{i}^{\prime \prime} A^{(i)}-$ произвольные линейные комбинации радиус-векторов вершин $A^{(i)}$ с постоянными коэфбициентами $\nu_{i}^{\prime}$ и $\nu_{i}^{\prime \prime} ;$ тогда функиця $\left\langle A^{\prime}, A^{\prime \prime}\right\rangle: M_{a_{\bar{i}}} \rightarrow \mathbb{C}$ полиномиальна по $p_{1}, q_{1}$, причем по $p_{1}-$ квадратична:

$$
\left\langle A^{\prime}, A^{\prime \prime}\right\rangle=p_{1}^{2} \mathscr{P}_{3}+2 p_{1} \mathscr{P}_{2}+\mathscr{P}_{1}
$$

здесь $\mathscr{P}_{k}$ - полиномы по q $q_{1}$ степени не выше " $k$ "; если степень $\mathscr{P}_{3}$ ниже трех, mo $\mathscr{P}_{2}=0, \mathscr{P}_{1}=$ const $_{\nu_{i}^{\prime}, \nu_{i}^{\prime \prime}}$.

Гамильтониан системы уравнений изомонодромных деформаций имеет именно такой вид - линейная комбинация $a_{i j}$; выпишем отдельно важную для нас формулу (здесь опять $t_{1}, t_{2}, t_{3}-$ произвольные числа)

$$
\begin{aligned}
a_{0 t}:= & t_{1} a_{01}+t_{2} a_{02}+t_{3} a_{03}=-\left(\sum t_{i} q_{i}\right) q_{1} q_{2} p_{1}^{2} \\
& +2 p_{1}\left(-\sqrt{\frac{a_{11}}{2}} q_{2}\left(\sum t_{i} q_{i}\right)+\sqrt{\frac{a_{22}}{2}} q_{1}\left(\sum t_{i} q_{i}\right)+\sqrt{\frac{a_{00}}{2}}\left(t_{2}-t_{1}\right) q_{1} q_{2}\right) \\
& +\left(\sum t_{i} q_{i}\right)\left(\left(\sqrt{\frac{a_{00}}{2}}+\sqrt{\frac{a_{11}}{2}}+\sqrt{\frac{a_{22}}{2}}\right)^{2}-\frac{a_{33}}{2}\right) \\
& +\sqrt{a_{00}}\left(t_{1} \sqrt{a_{11}}+t_{2} \sqrt{a_{22}}-t_{3}\left(\sqrt{a_{00}}+\sqrt{a_{11}}+\sqrt{a_{22}}\right)\right) .
\end{aligned}
$$

6.1. Рациональная (традиционная, классическая) форма $\mathrm{P}^{\mathrm{VI}}$. $\mathrm{B}$ первых разделах было показано, что условие изомонодромности деформации уравнения (1) задается формой $\Omega_{\mathrm{PvI}}$ :

$$
\Omega_{\mathrm{PVI}}=\omega-\sum_{i<j} d \operatorname{tr} A^{(i)} A^{(j)} \wedge d \log \left(\lambda_{i}-\lambda_{j}\right) .
$$

Мы знаем, что любая линейная комбинация величин $a_{i j}=\operatorname{tr} A^{(i)} A^{(j)}$ зависит квадратично от $p_{1}$ и кубично от $q_{1}$, где $\left(p_{1}, q_{1}\right)$ - симплектические координаты на $M_{a_{\bar{i}}}$ (в той части, где они определены), так что форма $\Omega_{\mathrm{Pvi}}$ в координатах $p_{1}, q_{1}$ перепишется так:

$$
d p_{1} \wedge d q_{1}-d\left(p_{1}^{2} \mathscr{P}_{3}+2 p_{1} \mathscr{P}_{2}+\mathscr{P}_{1}\right) \wedge d \tau
$$

Здесь $\mathscr{P}_{k}-$ многочлен степени $k$ от $q_{1}$, а $\tau$ - какой-нибудь параметр, от которого зависят $\lambda_{i}=\lambda_{i}(\tau)$; напомним, что изомонодромное распределение интегрируемо, результат эволюции решения не зависит от траектории, соединяющей начальную и конечную точки в пространстве параметров $\lambda_{i}$. Обозначим точкой производную по $\tau$. 
ТеОРема 11. Функиия $q_{1}=q_{1}(\tau)-q$-координата решения гамильтоновой системы (64) - удовлетворяет уравнению

$$
\ddot{q}_{1}=\frac{1}{2}\left(\frac{\partial}{\partial q_{1}} \log \mathscr{P}_{3}\right) \dot{q}_{1}^{2}+\left(\frac{\partial}{\partial \tau} \log \mathscr{P}_{3}\right) \dot{q}_{1}+2 \mathscr{P}_{3}\left(\frac{\partial}{\partial \tau} \frac{\mathscr{P}_{2}}{\mathscr{P}_{3}}+\frac{\partial}{\partial q_{1}}\left(\frac{\mathscr{P}_{2}^{2}}{\mathscr{P}_{3}}-\mathscr{P}_{1}\right)\right) .
$$

ДокАЗАТЕЛЬСтво. Выделим полный квадрат:

$$
p_{1}^{2} \mathscr{P}_{3}+2 p_{1} \mathscr{P}_{2}+\mathscr{P}_{1}=\mathscr{P}_{3}\left(p_{1}+\frac{\mathscr{P}_{2}}{\mathscr{P}_{3}}\right)^{2}+\mathscr{P}_{1}-\frac{\mathscr{P}_{2}^{2}}{\mathscr{P}_{3}}
$$

и введем новую переменную

$$
P:=p_{1}+\frac{\mathscr{P}_{2}}{\mathscr{P}_{3}}
$$

Форма примет вид

$$
d P \wedge d q_{1}-d\left(\mathscr{P}_{3} P^{2}+\mathscr{P}_{1}-\frac{\mathscr{P}_{2}^{2}}{\mathscr{P}_{3}}+R\right) \wedge d \tau
$$

Замена $p_{1} \rightarrow P$ явно содержит $\tau$, в гамильтониане появился новый член - это такая функция $R$, что $\left(\frac{\partial}{\partial \tau} \frac{\mathscr{P}_{2}}{\mathscr{P}_{3}}\right) d \tau \wedge d q_{1}=d R \wedge d \tau$, иначе

$$
\left(d R+\left(\frac{\partial}{\partial \tau} \frac{\mathscr{P}_{2}}{\mathscr{P}_{3}}\right) d q_{1}\right) \wedge d \tau=0, \quad \text { или } \quad \frac{\partial}{\partial q_{1}} R=-\frac{\partial}{\partial \tau} \frac{\mathscr{P}_{2}}{\mathscr{P}_{3}} .
$$

Функция $R=-\int\left(\frac{\partial}{\partial \tau} \frac{\mathscr{P}_{2}}{\mathscr{P}_{3}}\right) d q-$ первообразная от (рациональной) функции $-\frac{\partial}{\partial \tau} \frac{\mathscr{P}_{2}}{\mathscr{P}_{3}}$

Обозначим

$$
F=F\left(q_{1}, \tau\right):=\frac{\mathscr{P}_{2}^{2}}{\mathscr{P}_{3}}-\mathscr{P}_{1}+\int d q_{1} \frac{\partial}{\partial \tau} \frac{\mathscr{P}_{2}}{\mathscr{P}_{3}},
$$

система (64) приобрела простой вид

$$
d P \wedge d q_{1}-d\left(\mathscr{P}_{3} P^{2}-F\right) \wedge d \tau
$$

где $\mathscr{P}_{3}$ и $F$ не зависят от $P$. Выпишем каноническую систему:

$$
\dot{q}_{1}=2 P \mathscr{P}_{3}, \quad \dot{P}=-P^{2} \frac{\partial}{\partial q_{1}} \mathscr{P}_{3}+\frac{\partial}{\partial q_{1}} F .
$$

Исключим $P$ (это эквивалентно преобразованию Лежандра), переписав $P$ и $\dot{P}$ через $\dot{q}_{1}$ и $q_{1}$. Поскольку $2 P=\dot{q}_{1} / \mathscr{P}_{3}$, то

$$
\begin{aligned}
\ddot{q}_{1} & =-2\left(\frac{d}{d \tau} \mathscr{P}_{3}\right)\left(-\frac{1}{2} \frac{\dot{q}_{1}}{\mathscr{P}_{3}}\right)-2 \mathscr{P}_{3}\left(\left(\frac{1}{2} \frac{\dot{q}_{1}}{\mathscr{P}_{3}}\right)^{2} \frac{\partial}{\partial q_{1}} \mathscr{P}_{3}-\frac{\partial}{\partial q_{1}} F\right) \\
& =\frac{1}{2}\left(\frac{\partial}{\partial q_{1}} \log \mathscr{P}_{3}\right) \dot{q}_{1}^{2}+\left(\frac{\partial}{\partial \tau} \log \mathscr{P}_{3}\right) \dot{q}_{1}+2 \mathscr{P}_{3} \frac{\partial}{\partial q_{1}} F .
\end{aligned}
$$


ЗАмЕчаниЕ 22. Теорема объясняет структуру традиционной записи уравнения Пенлеве VI - три слагаемых специфического вида в правой части.

Введем параметр $t$ так, чтобы (65) приобрело привычный для специалистов вид (см. [1]).

Преобразуем сумму $\sum_{i<j} d a_{i j} \wedge d \log \left(\lambda_{i}-\lambda_{j}\right)$ :

$$
\begin{aligned}
\sum_{i<j} d a_{i j} \wedge d \log \left(\lambda_{i}-\lambda_{j}\right) & =\sum_{\substack{i=1 \\
j \neq i \neq k}}^{3} d a_{0 i} \wedge d \log \left[\left(\lambda_{0}-\lambda_{i}\right)\left(\lambda_{j}-\lambda_{k}\right)\right] \\
& =d\left(t_{1} a_{01}+t_{2} a_{02}+t_{3} a_{03}\right) \wedge d \tau \\
& =d\left(a_{01}+\frac{t_{3}-t_{2}}{t_{1}-t_{2}} a_{03}\right) \wedge\left(t_{1}-t_{2}\right) d \tau \\
& =d\left(a_{01}+t a_{03}\right) \wedge d \tau_{1-2}
\end{aligned}
$$

Здесь и ниже индексы $i, j, k$ в одной формуле все различны и отличны от индекса 0 , например, четная перестановка $\{1,2,3\}$. Мы обозначили:

а) логарифмическую производную величины $\left(\lambda_{0}-\lambda_{i}\right)\left(\lambda_{j}-\lambda_{k}\right)$ по параметру $\tau$ (произвольному, пока еще не определенному) через $t_{i}$ :

$$
t_{i}:=\frac{d \log \left[\left(\lambda_{0}-\lambda_{i}\right)\left(\lambda_{j}-\lambda_{k}\right)\right]}{d \tau}
$$

б) величину $\left(t_{3}-t_{2}\right) /\left(t_{1}-t_{2}\right)$ через $t$ :

$$
t:=\frac{t_{3}-t_{2}}{t_{1}-t_{2}}=\frac{d \log \left[\left(\lambda_{0}-\lambda_{3}\right)\left(\lambda_{1}-\lambda_{2}\right) /\left(\left(\lambda_{0}-\lambda_{2}\right)\left(\lambda_{3}-\lambda_{1}\right)\right)\right]}{d \log \left[\left(\lambda_{0}-\lambda_{1}\right)\left(\lambda_{2}-\lambda_{3}\right) /\left(\left(\lambda_{0}-\lambda_{2}\right)\left(\lambda_{3}-\lambda_{1}\right)\right)\right]} ;
$$

в) дифференциал $\left(t_{1}-t_{2}\right) d \tau$, имеющий вычет +1 в точке $\lambda_{0}=\lambda_{1}$, вычет -1 в точке $\lambda_{0}=\lambda_{2}$ и не имеющий никаких других особенностей (тут $\lambda_{1}$, $\lambda_{2}, \lambda_{3}$ фиксированы, а $\lambda_{0}-$ переменная), через $d \tau_{1-2}$ :

$$
d \tau_{1-2}:=d \log \frac{\left(\lambda_{0}-\lambda_{1}\right)\left(\lambda_{2}-\lambda_{3}\right)}{\left(\lambda_{0}-\lambda_{2}\right)\left(\lambda_{3}-\lambda_{1}\right)}=-d \log \mathscr{D}\left(\lambda_{1}, \lambda_{2}, \lambda_{3}, \lambda_{0}\right) .
$$

УтВеРЖДЕНИЕ 14. Через двойное отношение точек $\lambda_{i}$ дифберенциал $d \tau_{1-2}$ выражается так:

$$
d \tau_{1-2}=-\frac{d t}{t(t-1)}, \quad t=\frac{\left(\lambda_{0}-\lambda_{1}\right)\left(\lambda_{2}-\lambda_{3}\right)}{\left(\lambda_{0}-\lambda_{3}\right)\left(\lambda_{2}-\lambda_{1}\right)}=\mathscr{D}\left(\lambda_{1}, \lambda_{3}, \lambda_{0}, \lambda_{2}\right) .
$$

Это является следствием двух красивых формул.

УтВеРЖДЕНИЕ 15. Для любых величин $s_{1}, s_{2}, s_{3}$ таких, что $s_{1}+s_{2}+s_{3}=0$, выполнено равенство

$$
d \log \frac{s_{1}}{s_{2}}+d \log \frac{d \log \left(s_{1} / s_{3}\right)}{d \log \left(s_{2} / s_{3}\right)}=0 .
$$


ДокАЗАТЕЛЬСтво. Введем переменную $x:=s_{1} / s_{2}$. Тогда

$$
\frac{s_{1}}{s_{3}}=-\frac{s_{1}}{s_{1}+s_{2}}=-\frac{x}{x+1}, \quad \frac{s_{2}}{s_{3}}=-\frac{1}{x+1} .
$$

УтВеРЖДЕНИЕ 16. Для любъх $\lambda_{0}, \lambda_{1}, \lambda_{2}, \lambda_{3}$

$$
\left(\lambda_{0}-\lambda_{1}\right)\left(\lambda_{2}-\lambda_{3}\right)+\left(\lambda_{0}-\lambda_{2}\right)\left(\lambda_{3}-\lambda_{1}\right)+\left(\lambda_{0}-\lambda_{3}\right)\left(\lambda_{1}-\lambda_{2}\right)=0
$$

ДокАЗАтЕльство. Эта сумма обращается в нуль, если положить $\lambda_{i}=\lambda_{j}$, значит, она делится на $\lambda_{i}-\lambda_{j}$ для любых $i, j$. В то же время все выражение квадратично по $\lambda_{j}$, а различных пар $\lambda_{i}-\lambda_{j}$ больше двух (шесть).

Утверждение 14 доказывается так.

В утверждении 15 положим $s_{i}=\left(\lambda_{0}-\lambda_{i}\right)\left(\lambda_{j}-\lambda_{k}\right)$ и выразим $t$ и $d \tau_{1-2}$ через $x=s_{1} / s_{2}$ :

$$
\begin{aligned}
& d \tau_{1-2}=d \log x \\
& t=\frac{d \log \left(s_{3} / s_{2}\right)}{d \log \left(s_{1} / s_{2}\right)}=\frac{d \log (1+x)}{d \log x}=\frac{x}{1+x}=-\frac{s_{1}}{s_{3}} \\
& =-\frac{\left(\lambda_{0}-\lambda_{1}\right)\left(\lambda_{2}-\lambda_{3}\right)}{\left(\lambda_{0}-\lambda_{3}\right)\left(\lambda_{1}-\lambda_{2}\right)}=\mathscr{D}\left(\lambda_{1}, \lambda_{3}, \lambda_{0}, \lambda_{2}\right), \\
& t=\frac{x}{1+x} \Leftrightarrow x=\frac{t}{1-t} \Rightarrow d \tau_{1-2}=d \log \frac{t}{t-1}=\left(\frac{1}{t}-\frac{1}{t-1}\right) d t=-\frac{d t}{t(t-1)} .
\end{aligned}
$$

Вернемся к уравнению Пенлеве VI. Величина $a_{01}+t a_{03}$ выражается через $p_{1}, q_{1}$ с помощью (63). Если в качестве независимой переменной ${ }^{5} \tau$ в формуле (64) выбрать

$$
\tau:=t=\frac{t_{3}-t_{2}}{t_{1}-t_{2}}=\mathscr{D}\left(\lambda_{1}, \lambda_{3}, \lambda_{0}, \lambda_{2}\right),
$$

то получим

$$
\begin{aligned}
\sum_{i<j} d a_{i j} \wedge d \log \left(\lambda_{i}-\lambda_{j}\right)=d\left(a_{01}+t a_{03}\right) \wedge \frac{-d t}{t(t-1)} \\
=d \frac{-1}{t(t-1)}\left(t_{1} a_{01}+t_{2} a_{02}+t_{3} a_{03}\right) \wedge d t=d\left(p_{1}^{2} \mathscr{P}_{3}+2 p_{1} \mathscr{P}_{2}+\mathscr{P}_{1}\right) \wedge d t \\
=d \frac{-1}{t(t-1)}\left(-\left(\sum t_{i} q_{i}\right) q_{1} q_{2} p_{1}^{2}+2 p_{1}\left(-\sqrt{\frac{a_{11}}{2}} q_{2}\left(\sum t_{i} q_{i}\right)\right.\right. \\
\left.+\sqrt{\frac{a_{22}}{2}} q_{1}\left(\sum t_{i} q_{i}\right)+\sqrt{\frac{a_{00}}{2}}\left(t_{2}-t_{1}\right) q_{1} q_{2}\right) \\
\left.+\left(\sum t_{i} q_{i}\right)\left(\left(\sqrt{\frac{a_{00}}{2}}+\sqrt{\frac{a_{11}}{2}}+\sqrt{\frac{a_{22}}{2}}\right)^{2}-\frac{a_{33}}{2}\right)\right) \wedge d t
\end{aligned}
$$

\footnotetext{
${ }^{5} \mathrm{~B}$ следующем разделе и $\tau$ и $\mathscr{P}_{i}$ будут другими.
} 
и функции $\mathscr{P}_{3}, \mathscr{P}_{2}$ и $\mathscr{P}_{1}$ из $(64)$, участвующие в формулировке теоремы 11 , получаются такими:

$$
\begin{aligned}
& \mathscr{P}_{3}=-\frac{q_{1} q_{2} q_{t}}{t(t-1)}, \\
& \mathscr{P}_{2}=\frac{q_{1} q_{2} q_{t}}{t(t-1)}\left(-\frac{\sqrt{a_{11} / 2}}{q_{1}}+\frac{\sqrt{a_{22} / 2}}{q_{2}}+\frac{\sqrt{a_{00} / 2}}{q_{t}}\right) \\
& \mathscr{P}_{1}=\frac{q_{t}}{t(t-1)}\left(\left(\sqrt{\frac{a_{00}}{2}}+\sqrt{\frac{a_{11}}{2}}+\sqrt{\frac{a_{22}}{2}}\right)^{2}-\frac{a_{33}}{2}\right)+\text { const }_{t} .
\end{aligned}
$$

Мы обозначили $q_{t}:=-\left(q_{1}+t\right)=-\sum q_{i} t_{i}$, так что $q_{1}+q_{t}+t=q_{t}+q_{2}+1=0$; величину const $t$, не зависящую от $q_{1}$ (и от $p_{1}$, конечно, тоже), больше писать не будем.

Функция

$$
\begin{aligned}
F:= & \frac{\mathscr{P}_{2}^{2}}{\mathscr{P}_{3}}-\mathscr{P}_{1}+\int d q_{1} \frac{\partial}{\partial \tau} \frac{\mathscr{P}_{2}}{\mathscr{P}_{3}} \\
=- & \frac{q_{1}\left(q_{1}+1\right)\left(q_{1}+t\right)}{t(t-1)}\left(\frac{\sqrt{a_{11} / 2}}{q_{1}}+\frac{\sqrt{a_{22} / 2}}{q_{1}+1}+\frac{\sqrt{a_{00} / 2}}{q_{1}+t}\right)^{2} \\
& \quad+\frac{q_{1}+t}{t(t-1)}\left(\left(\sqrt{\frac{a_{00}}{2}}+\sqrt{\frac{a_{11}}{2}}+\sqrt{\frac{a_{22}}{2}}\right)^{2}-\frac{a_{33}}{2}\right)+\frac{\sqrt{a_{00} / 2}}{q_{1}+t}
\end{aligned}
$$

имеет только простые полюсы на $\mathbb{C} \ni q_{1}$; вычислим вычеты:

$$
\begin{gathered}
\left.\operatorname{Res}\right|_{q_{1}=0} F=-\frac{a_{11}}{2} \frac{1}{t-1},\left.\quad \operatorname{Res}\right|_{q_{1}=-1} F=\frac{a_{22}}{2} \frac{1}{t}, \\
\left.\operatorname{Res}\right|_{q_{1}=-t} F=-\frac{a_{00}}{2}+\sqrt{\frac{a_{00}}{2}},\left.\quad \operatorname{Res}\right|_{q_{1}=\infty} F=-\frac{a_{33}}{2} \frac{1}{t(t-1)},
\end{gathered}
$$

так что

$$
F=\frac{1}{2 t(t-1)}\left(-\frac{t a_{11}}{q_{1}}+\frac{(t-1) a_{22}}{q_{1}+1}-\frac{t(t-1)\left(a_{00}-\sqrt{2 a_{00}}\right)}{q_{1}+t}-a_{33} q_{1}\right)+\text { const }_{t}^{\prime}
$$

и, следовательно,

$$
\begin{gathered}
\frac{\partial}{\partial q_{1}} \log \mathscr{P}_{3}=\frac{1}{q_{1}}+\frac{1}{q_{1}+1}+\frac{1}{q_{1}+t}, \\
\frac{\partial}{\partial t} \log \mathscr{P}_{3}=-\left(\frac{1}{t}+\frac{1}{t-1}-\frac{1}{t+q_{1}}\right) \\
2 \mathscr{P}_{3}\left(\frac{\partial}{\partial \tau} \frac{\mathscr{P}_{2}}{\mathscr{P}_{3}}-\frac{\partial}{\partial q_{1}}\left(\frac{\mathscr{P}_{2}^{2}}{\mathscr{P}_{3}}-\mathscr{P}_{1}\right)\right)=2 \mathscr{P}_{3} \frac{\partial}{\partial q_{1}} F \\
=-\frac{q_{1}\left(q_{1}+1\right)\left(q_{1}+t\right)}{t^{2}(t-1)^{2}}\left(a_{11} \frac{t}{q_{1}^{2}}-a_{22} \frac{t-1}{\left(q_{1}+1\right)^{2}}\right. \\
\left.\quad+\left(a_{00}-\sqrt{2 a_{00}}\right) \frac{t(t-1)}{\left(q_{1}+t\right)^{2}}-a_{33}\right) .
\end{gathered}
$$


На функцию $q:=-q_{1}$ мы получили (формула (65)) уравнение Пенлеве VI с параметрами $\alpha=a_{33}, \beta=a_{22}, \gamma=a_{11}, \delta=\left(\sqrt{a_{00}}-1 / \sqrt{2}\right)^{2}$ :

$$
\begin{aligned}
\ddot{q}=\frac{1}{2} & \left(\frac{1}{q}+\frac{1}{q-1}+\frac{1}{q-t}\right) \dot{q}^{2}-\left(\frac{1}{t}+\frac{1}{t-1}+\frac{1}{q-t}\right) \dot{q} \\
& +\frac{q(q-1)(q-t)}{t^{2}(t-1)^{2}}\left(\alpha-\beta \frac{t}{q^{2}}+\gamma \frac{t-1}{(q-1)^{2}}+\left(\frac{1}{2}-\delta\right) \frac{t(t-1)}{(q-t)^{2}}\right) .
\end{aligned}
$$

6.2. Эллиптическая форма $\mathrm{P}^{\mathrm{VI}}$. В этом пункте активно используются эллиптические функции, для ознакомления с предметом автор рекомендует книгу [41], сочетающую в себе свойства как учебника, так и справочника.

На протяжении этого пункта будем писать $q_{0}$ вместо $q_{t}$; также в качестве независимой переменной $\tau$ будет выбрана другая функция $\tau=\tau\left(\lambda_{0}, \lambda_{1}, \lambda_{2}, \lambda_{3}\right)$, не $t=\mathscr{D}\left(\lambda_{1}, \lambda_{3}, \lambda_{0}, \lambda_{2}\right)$, так что изменятся (исчезнет $t(t-1)$ в знаменателе) $\mathscr{P}_{3}$ и $F$.

Итак, отныне

$$
\begin{gathered}
\Omega_{\mathrm{PVI}}=d P \wedge d q_{1}-d\left(\mathscr{P}_{3} P^{2}-F\right) \wedge d \tau_{1-2}, \\
P=p_{1}+\frac{\sqrt{a_{11} / 2}}{q_{1}}-\frac{\sqrt{a_{22} / 2}}{q_{2}}-\frac{\sqrt{a_{00} / 2}}{q_{0}}, \quad q_{0}:=q_{t}:=-\left(q_{1}+t\right), \\
\mathscr{P}_{3}=q_{1} q_{2} q_{t}, \quad d \tau_{1-2}=-\frac{d t}{t(t-1)}, \quad t=\mathscr{D}\left(\lambda_{1}, \lambda_{3}, \lambda_{0}, \lambda_{2}\right) \\
F=\frac{a_{11}}{2}+\frac{a_{11}}{2}(t-1) q_{2}-\left(\frac{a_{00}}{2}-\sqrt{\frac{a_{00}}{2}}\right) \frac{t(t-1)}{q_{0}}+\frac{a_{33}}{2} q_{1} .
\end{gathered}
$$

Обозначим через $Q_{i} \in L_{Q}^{0}$ корни $q_{i}: q_{i}\left(Q_{i}\right)=0$; функция $q_{i}$ обращается в нуль в точке $Q_{i}$ (точка $Q_{3}$ - это та точка, где $q_{1}, q_{2}$ и $q_{0}$ имеют полюс). Получим

$$
\begin{gathered}
F=\frac{a_{11}}{2} \frac{q_{1}\left(Q_{2}\right) q_{1}\left(Q_{0}\right)}{q_{1}}-\frac{a_{22}}{2} \frac{q_{2}\left(Q_{1}\right) q_{2}\left(Q_{0}\right)}{q_{2}}+\frac{a_{33}}{2} q_{1} \\
-\left(\frac{a_{00}}{2}-\sqrt{\frac{a_{00}}{2}}\right) \frac{q_{0}\left(Q_{1}\right) q_{0}\left(Q_{2}\right)}{q_{0}} .
\end{gathered}
$$

Рассмотрим дробно-линейное преобразование $\chi_{k}, k \in\{1,2,0\}$, сферы Римана $L_{Q}^{0}$, переводящее точку $Q_{3}$ в $Q_{k}$ и меняющее местами оставшиеся точки $Q_{i}$ и $Q_{j}$.

УтВеРЖДЕНИЕ 17. На функцию $q_{k}$ преобразование $\chi_{k}$ действует следующим образом:

$$
\chi_{k}^{*} q_{k}=\frac{q_{k}\left(Q_{i}\right) q_{k}\left(Q_{j}\right)}{q_{k}}, \quad i \neq j \neq k \neq i .
$$

ДоказАтельство. Рациональная функция $\chi_{k}^{*} q_{k}$ с единственной особенностью - простым полюсом принимает те же значения, что и $q_{k}\left(Q_{i}\right) q_{k}\left(Q_{j}\right) / q_{k}$, в четырех различных точках $Q_{l}, l \in\{0,1,2,3\}$. 
СледСтвиЕ 4. Функиия $F$ следующим образом выражается через коордиramy $q_{1}$ :

$$
F=\frac{a_{11}}{2} \chi_{1}^{*} q_{1}+\frac{a_{22}}{2} \chi_{2}^{*} q_{1}+\frac{a_{33}}{2} q_{1}+\left(\frac{a_{00}}{2}-\sqrt{\frac{a_{00}}{2}}\right) \chi_{0}^{*} q_{1}+\text { const }_{\tau},
$$

где const $_{\tau}-$ постоянная, т.е. не зависит от точки $L_{Q}$.

ДокАЗАтЕЛЬство. Рассмотрим действие $\chi_{k}$ на $q_{1}$ :

$$
\begin{aligned}
& \chi_{2}^{*} q_{1}=-\chi_{2}^{*}\left(q_{2}+1\right)=-\frac{q_{2}\left(Q_{1}\right) q_{2}\left(Q_{0}\right)}{q_{2}}-1 \Longrightarrow-\frac{1}{q_{2}}=\left(\chi_{2}^{*} q_{1}+1\right) \frac{1}{q_{2}\left(Q_{1}\right) q_{2}\left(Q_{0}\right)}, \\
& \chi_{0}^{*} q_{1}=-\chi_{2}^{*}\left(q_{0}+t\right)=-\frac{q_{0}\left(Q_{1}\right) q_{0}\left(Q_{2}\right)}{q_{0}}-t \Longrightarrow-\frac{1}{q_{0}}=\left(\chi_{0}^{*} q_{1}+t\right) \frac{1}{q_{0}\left(Q_{1}\right) q_{0}\left(Q_{2}\right)} .
\end{aligned}
$$

Постараемся избавиться от множителя $\mathscr{P}_{3}$ перед $P^{2}$ - перейти к таким переменным $u, p, \tau$, чтобы зависимость гамильтониана от импульса стала чисто квадратичной:

$$
\Omega_{\mathrm{PVI}}=d p \wedge d u-d\left(\frac{p^{2}}{2}-\mathscr{F}(u, \tau)\right) \wedge \frac{d \tau}{\mathrm{const}}=0 .
$$

Каноническая система в этом случае упростится:

$$
\text { const } \frac{d u}{d \tau}=p, \quad \text { const } \frac{d p}{d \tau}=\frac{\partial}{\partial u} \mathscr{F}(u, \tau),
$$

и уравнение на $u$ (уравнение Пенлеве VI) примет вид

$$
(\text { const })^{2} \frac{d^{2}}{d \tau^{2}} u=\mathscr{F}^{\prime}(u, \tau), \quad \mathscr{F}^{\prime}:=\left.\frac{\partial}{\partial u}\right|_{\tau} \mathscr{F}(u, \tau) .
$$

Введем в (69) (временно) переменную $\widetilde{P}:=\sqrt{\mathscr{P}_{3}} P$. Она определена на двулистном накрытии сферы Римана $\overline{\mathbb{C}} \ni q_{1}$, разветвленном в нулях $\mathscr{P}_{3}: \mathscr{P}_{3}=$ $q_{1} q_{2} q_{0}=q_{1}\left(q_{1}+1\right)\left(q_{1}+t\right)$ и в бесконечности, т. е. определена на торе, модулярный параметр которого зависит от независимой переменной $t$. Построим координату $\tilde{v}$, канонически сопряженную $\widetilde{P}$ (т. е. $\left.\widetilde{P} d \tilde{v}=P d q_{1}\right)$ :

$$
d \tilde{v}=\frac{d q_{1}}{\sqrt{q_{1}\left(q_{1}+1\right)\left(q_{1}+t\right)}} .
$$

Дифференциал $d \tilde{v}$ - голоморфный дифференциал на торе, сама же переменная $\tilde{v}:=\int d \tilde{v}$ оказывается голоморфной координатой на универсальной накрывающей этого тора - на комплексной плоскости.

Прежде чем приступить к реализации этой схемы, заметим, что выбранный нами вид (71) формы $\Omega_{\mathrm{PVI}}$ очень “жесткий” - он оставляет возможность только линейных относительно $u$ замен переменных, однозначно связанных с заменой независимой переменной $\tau$ :

$$
(u, d \tau) \rightarrow\left(\varkappa_{1} u+\varkappa_{0}, c \varkappa_{1}^{2} d \tau\right), \quad \varkappa_{1}=\varkappa_{1}(\tau), \quad \varkappa_{0}=\varkappa_{0}(\tau)
$$


При данном преобразовании $\mathscr{F}^{\prime}$ меняется линейно по $u$ :

$$
\mathscr{F}^{\prime} \mapsto u \varkappa_{1} \frac{d^{2}}{d \tau^{2}}\left(\frac{1}{\varkappa_{1}}\right)-\varkappa_{1} \frac{d}{d \tau}\left(\frac{1}{\varkappa_{1}^{2}} \frac{d \varkappa_{0}}{d \tau}\right)+c^{2} \varkappa_{1}^{3} \mathscr{F}^{\prime}
$$

(см. [42]). Если потребовать, чтобы $\mathscr{F}(u, \tau)$ при постоянном $\tau$ оставалась функцией на $L_{Q}^{0}$ (определялась тетраэдром $\left.A^{(\bar{i})}\right)$, т. е. была двояко-периодической функцией на торе, вся свобода изменения независимой переменной $\tau$ сведется к умножению на константу и модулярному преобразованию $\tau \rightarrow-1 / \tau$. Потребуем, чтобы образ какой-нибудь фиксированной точки $\mathbb{C}_{u}$, например $u=0$, совпал с одной из точек $Q_{j} \in L_{Q}^{0}$. В этом случае $\varkappa_{0}$ должно быть вектором решетки периодов тора и при выборе переменных $(u, \tau)$ не останется ни одного функционального параметра.

Таким образом, предложенный вид (71) формы $\Omega_{\mathrm{PvI}}$, с точностью до дискретных симметрий и тривиальных (не зависящих от переменных $u, p$ и $\tau$ ) масштабных преобразований, определяет как зависимую переменную $u$, так и независимую переменную $\tau$.

ТЕОРема 12. Функиия $\mathscr{F}=\mathscr{F}(u, \tau)$ в (71) тогда и только тогда может быть опущена на $L_{Q}^{0}$, когда один из периодов постоянен, т.е. существует контур интегрирования такой, что $\oint u=\mathrm{const,} \mathrm{a} \tau$ пропориионально второму примитивному периоду - меняется на верхней полуплоскости, на универсальной накрывающей $\overline{\mathbb{C}} \backslash\{0,1, \infty\} \ni t$.

ДокАзАтельство. Ниже мы докажем, что указанный выбор $u$ и $\tau$ действительно дает $\mathscr{F}$, совпадающую с поднятием на $\mathbb{C}_{u}$ некоторой рациональной на $L_{Q}^{0}$ функции. Доказательство же необходимости (такого выбора) следует из приведенного выше рассуждения.

Пусть $T_{t}-$ тор (погруженный в $\overline{\mathbb{C}} \times \overline{\mathbb{C}}$ ), униформизованный двумя переменными $\sqrt{\mathscr{P}_{3}}$ и $q_{1}$, связанными соотношением

$$
\left(\sqrt{\mathscr{P}_{3}}\right)^{2}=q_{1}\left(q_{1}+1\right)\left(q_{1}+t\right)
$$

Пусть $u$ - голоморфная переменная на его универсальной накрывающей $\mathbb{C}_{u}$. Прообразы точек $Q_{j}, j \in\{0,1,2,3\}$ :

$$
q_{1}\left(Q_{1}\right)=0, \quad q_{1}\left(Q_{2}\right)=-1, \quad q_{1}\left(Q_{3}\right)=\infty, \quad q_{1}\left(Q_{0}\right)=-t
$$

при накрывающем отображении $\pi_{u T}: \mathbb{C} \rightarrow T_{t}$ образуют решетку полупериодов на $\mathbb{C}_{u}$.

Выберем в качестве точки $u=0$ (и обозначим $\omega_{0}:=0$ ) один из узлов решетки, соответствующих $Q_{0}$; остальные полупериоды занумеруем в соответствии с обозначениями Вейерштрасса:

$$
\omega_{j}:=\operatorname{const}(t) \int_{q_{1}\left(Q_{0}\right)}^{q_{1}\left(Q_{j}\right)} \frac{d q}{\sqrt{\mathscr{P}_{3}}},
$$

так что $\omega_{1}+\omega_{2}+\omega_{3}=0, \operatorname{Im}\left(\omega_{3} / \omega_{1}\right)>0$. 
ТеОрема 13. Пусть $\tau:=\omega_{3} / \omega_{1}$ - отношение примитивных периодов, соответствующих $Q_{3}$ и $Q_{1}$, тогда

$$
\left(\int_{q_{1}\left(Q_{0}\right)}^{q_{1}\left(Q_{1}\right)} \frac{d q_{1}}{\sqrt{\mathscr{P}_{3}}}\right)^{2}=4 \pi i \frac{d \tau_{1-2}}{d \tau}=4 \pi i \frac{d \log \mathscr{D}\left(\lambda_{1}, \lambda_{2}, \lambda_{0}, \lambda_{3}\right)}{d \tau} .
$$

ДокАзАтЕльство. Это - некоторое тождество, содержащее полные эллиптические интегралы. Оно следует из формул (81), (82) и (80), которые нам будет удобнее доказать позже, когда выразим $q_{1}, \sqrt{\mathscr{P}_{3}}$ и $d \tau_{1-2} / d \tau$ через стандартные функции на торе.

Пусть переменная $u$ на $\mathbb{C}_{u}$ выбрана так, что $2 \omega_{1}=1$, т. е.

$$
\left.d\right|_{\tau=\text { const }} u=\frac{d q_{1}}{\sqrt{\mathscr{P}_{3}}} / \sqrt{4 \pi i \frac{d \tau_{1-2}}{d \tau}}, \quad \text { или } \quad u=\int \frac{d q}{\sqrt{\mathscr{P}_{3}}} / \sqrt{4 \pi i \frac{d \tau_{1-2}}{d \tau}} .
$$

Интеграл в правой части явно зависит от $\tau$. Эту зависимость охарактеризуем функцией $\phi=\phi(u, \tau)$ :

$$
\begin{gathered}
\phi:=-\left.2 \pi i \frac{\partial}{\partial \tau}\right|_{q_{1}=\mathrm{const}} u, \quad d u=\left.\frac{\partial u}{\partial q_{1}}\right|_{\tau} d q_{1}+\left.\frac{\partial u}{\partial \tau}\right|_{q_{1}} d \tau \\
d q_{1}=\left(\left.\frac{\partial q_{1}}{\partial u}\right|_{\tau}\right)\left(d u+\frac{\phi}{2 \pi i} d \tau\right) .
\end{gathered}
$$

Вследствие нормировки (75)

$$
\left.\frac{\partial q_{1}}{\partial u}\right|_{\tau}=\sqrt{\mathscr{P}_{3}} \sqrt{4 \pi i \frac{d \tau_{1-2}}{d \tau}}
$$

значит,

$$
d q_{1}=\sqrt{\mathscr{P}_{3}} \sqrt{4 \pi i \frac{d \tau_{1-2}}{d \tau}}\left(d u+\frac{\phi}{2 \pi i} d \tau\right)
$$

Подставим это выражение в $(69)$ и обозначим $\hat{p}:=P \sqrt{\mathscr{P}_{3}} \sqrt{4 \pi i \frac{d \tau_{1-2}}{d \tau}}$ :

$$
\begin{aligned}
\Omega_{\mathrm{PVI}} & =d\left(P d q_{1}\right)-d\left(\mathscr{P}_{3} P^{2}-F\right) \wedge d \tau_{1-2} \\
& =d\left(\widehat{P}\left(d u+\frac{\phi}{2 \pi i} d \tau\right)\right)-d\left(\frac{\hat{p}^{2}}{4 \pi i d \tau_{1-2} / d \tau}-F\right) \wedge d \tau_{1-2} \\
& =d(\hat{p} d u)+d(2 \hat{p} \phi) \wedge \frac{d \tau}{4 \pi i}-d\left(\hat{p}^{2}-4 \pi i \frac{d \tau_{1-2}}{d \tau} F\right) \wedge \frac{d \tau}{4 \pi i} \\
& =d(\hat{p} d u)-d\left((\hat{p}-\phi)^{2}-\phi^{2}-4 \pi i \frac{d \tau_{1-2}}{d \tau} F\right) \wedge \frac{d \tau}{4 \pi i} \\
& =d p \wedge d u-d\left(\frac{p^{2}}{2}-2 \pi i \frac{d \tau_{1-2}}{d \tau} F\right) \wedge \frac{d \tau}{2 \pi i}+d \phi \wedge d u+d \phi^{2} \wedge \frac{d \tau}{4 \pi i} .
\end{aligned}
$$

Здесь мы обозначили

$$
p:=\hat{p}-\phi=P \sqrt{\mathscr{P}_{3}} \sqrt{4 \pi i \frac{d \tau_{1-2}}{d \tau}}+\left.2 \pi i \frac{\partial}{\partial \tau}\right|_{q_{1}} u
$$


Далее будем обозначать производную по $u$ при постоянном $\tau$ штрихом; дифференцирование $\partial / \partial \tau$ всегда будет при постоянном $u$ :

$$
\ldots ':=\left.\frac{\partial}{\partial u}\right|_{\tau=\text { const }} \ldots, \quad \frac{\partial}{\partial \tau} \ldots=\left.\frac{\partial}{\partial \tau}\right|_{u=\text { const }} \ldots .
$$

Отображение комплексной плоскости $\mathbb{C}_{u} \ni u$ в сферу Римана $L_{Q}^{0}$, находящуюся во взаимно однозначном соответствии со значениями функции $q_{1}$, обозначим $\pi_{u Q}$.

При обозначении тэта-функций модулярный аргумент $\tau$ будем опускать; если $u$ - первый аргумент тэта-функции не указан, то он равен нулю:

$$
\theta_{k}(u):=\theta_{k}(u \mid \tau), \quad \theta_{k}:=\theta_{k}(0 \mid \tau), \quad \theta_{k}^{\prime}:=\left.\frac{\partial}{\partial u} \theta_{k}(u \mid \tau)\right|_{u=0} .
$$

Связь нумерации полупериодов Вейерштрасса и индексов в тэта-функциях:

$$
0=\theta_{k+1}\left(\frac{\omega_{k}}{2 \omega_{1}}\right) \quad \forall k \in \mathbb{Z} \quad(\bmod 4), \quad \omega_{0} \stackrel{\text { def }}{=} 0 .
$$

Все тэта-функции удовлетворяют уравнению теплопроводности

$$
4 \pi i \frac{\partial}{\partial \tau} \theta_{k}(u \mid \tau)=\frac{\partial^{2}}{\partial u^{2}} \theta_{k}(u \mid \tau) .
$$

Разделив обе части на $\theta_{k}(u \mid \tau)$, получим версию этого уравнения для логарифма тэта-функции:

$$
4 \pi i \frac{\partial}{\partial \tau} \log \theta_{k}(u)=\log ^{\prime \prime} \theta_{k}(u)+\left(\log ^{\prime} \theta_{k}(u)\right)^{2} .
$$

Вычислим $d \tau_{1-2} / d \tau$. По определению, $d \tau_{1-2}=d \log \mathscr{D}\left(\lambda_{1}, \lambda_{2}, \lambda_{0}, \lambda_{3}\right)$. Двойное отношение не зависит от того, какая именно голоморфная переменная на $\mathbb{C P}^{1}$ используется для его вычисления - любые две координаты связаны дробно-линейным преобразованием, от которого двойное отношение не зависит. Из соображений удобства выберем $\hat{q}=\theta_{1}^{2}(u) / \theta_{0}^{2}(u)$, тогда

$$
d \tau_{1-2}=d \log \mathscr{D}\left(\lambda_{1}, \lambda_{2}, \lambda_{0}, \lambda_{3}\right)=d \log \mathscr{D}\left(\hat{q}\left(Q_{1}\right), \hat{q}\left(Q_{2}\right), \hat{q}\left(Q_{0}\right), \hat{q}\left(Q_{3}\right)\right),
$$

где $\hat{q}\left(Q_{j}\right):=\theta_{1}^{2}\left(\omega_{j}\right) / \theta_{0}^{2}\left(\omega_{j}\right)$. Поскольку $\theta_{0}^{2}\left(\omega_{3}\right)=\theta_{1}^{2}\left(\omega_{0}\right)=0$, то

$$
\begin{aligned}
d \tau_{1-2} & =d \log \frac{\theta_{1}^{2}(1 / 2) \theta_{0}^{2}((1+\tau) / 2)}{\theta_{1}^{2}((1+\tau) / 2) \theta_{0}^{2}(1 / 2)} \\
& =d \log \frac{\theta_{2}^{4}}{\theta_{3}^{4}}=\frac{1}{\pi i}\left(4 \pi i \frac{\partial}{\partial \tau} \log \frac{\theta_{2}}{\theta_{3}}\right) d \tau .
\end{aligned}
$$

Воспользуемся формулой (79). Учитывая, что $\log ^{\prime} \theta_{2}=\log ^{\prime} \theta_{3}=0$, получим

$$
\begin{aligned}
\frac{d \tau_{1-2}}{d \tau} & =\left.\frac{1}{\pi i} \frac{\log ^{\prime \prime} \theta_{2}(u)}{\theta_{3}(u)}\right|_{u=0}=\left.\frac{(2 K)^{2}}{\pi i} \frac{d^{2}}{d v^{2}} \log \operatorname{sn}(v+K)\right|_{v=0} \\
& =\left.\frac{4 K^{2}}{\pi i} \frac{d}{d v}\right|_{v=0} \frac{\operatorname{cn}(v+K) \operatorname{dn}(v+K)}{\operatorname{sn}(v+K)}=\left.\frac{4 K^{2}}{\pi i} \frac{d}{d v}\right|_{v=0}\left(-k^{2}\right) \frac{\operatorname{sn} v \operatorname{dn} v}{\operatorname{dn}^{2} v \operatorname{cn} v} \\
& =\left.\frac{\pi^{2} \theta_{3}^{4}}{\pi i} \frac{\theta_{0}^{4}}{\theta_{3}^{4}}(-1) \frac{\operatorname{cn} v \operatorname{dn} v}{\operatorname{dn} v \operatorname{cn} v}\right|_{v=0}=i \pi \theta_{0}^{4}
\end{aligned}
$$


Итак, значение $d \tau_{1-2} / d \tau$ вычислено, положим

$$
\sqrt{4 \pi i \frac{d \tau_{1-2}}{d \tau}}:=2 \pi i \theta_{0}^{2} .
$$

Рассмотрим автоморфизм $\sigma_{\omega_{k}}: \mathbb{C}_{u} \rightarrow \mathbb{C}_{u}$, состоящий в сдвиге на полупериод $\omega_{k}: \sigma_{\omega_{k}}(u)=u+\omega_{k}, k \in\{1,2,3\}$.

При этом преобразовании полупериоды переходят друг в друга и ни один не остается на месте; кроме того, точки, симметричные относительно инволюции $u \rightarrow-u$, переходят опять в симметричные же точки, следовательно, эта инволюция опускается на $L_{Q}^{0}$.

Легко понять, что

$$
\left(\pi_{u Q}^{*}\right)^{-1} \sigma_{\left(\omega_{k}-\omega_{3}\right)}=\chi_{k}
$$

где обозначено $\sigma_{\left(\omega_{k}-\omega_{3}\right)}(u):=u+\omega_{k}-\omega_{3}$.

Из этого следует, что в формуле $(70)$ для $F$ при различных $a_{i i} / 2$ стоит одна и та же функция переменной $u$, например $\pi_{u Q}^{*} q_{1}$, сдвинутая на различные полупериоды $\omega_{j}$. Вычислим $\pi_{u Q}^{*} q_{1}-$ выразим ее через стандартные эллиптические функции.

Top $T_{t}$, заданный уравнением $(73)$, двулистно накрывает $L_{Q}^{0}$; точки $L_{Q}^{0}$ находятся в биективном соответствии со значениями функции $q_{1}: L_{Q}^{0} \rightarrow \overline{\mathbb{C}}$ :

$$
\pi_{T Q}: T_{t} \rightarrow L_{Q}^{0}
$$

Это накрытие разветвлено над точками $Q_{k}, k \in\{0,1,2,3\}$. На $T_{t}$ определены: функции $q_{i}$ (поднятия соответствующих функций на $L_{Q}^{0}$ ), функция $\sqrt{\mathscr{P}_{3}}$, проекция $\left(q_{1}, \sqrt{\mathscr{P}_{3}}\right) \rightarrow q_{1}$ и инволюция $u \rightarrow-u$, переставляющая листы накрытия $\left(q_{1}, \sqrt{\mathscr{P}_{3}}\right) \leftrightarrows\left(q_{1},-\sqrt{\mathscr{P}_{3}}\right)$. Плоскость $\mathbb{C}_{u}$ является универсальной накрывающей тора $T_{t}$ :

$$
\pi_{u T}: \mathbb{C}_{u} \rightarrow T_{t}
$$

Это отображение является бесконечнолистным неразветвленным накрытием.

Композиция этих двух накрывающих отображений -

$$
\pi_{u T}: \mathbb{C}_{u} \rightarrow T_{t} \quad \text { и } \quad \pi_{T Q}: T_{t} \rightarrow L_{Q}^{0}
$$

обозначена была нами через $\pi_{u Q}$ :

$$
\pi_{u Q}: \mathbb{C}_{u} \rightarrow L_{Q}^{0}, \quad \pi_{u Q}=\pi_{T Q} \circ \pi_{u T}
$$

Отображение $\pi_{u Q}$ - бесконечнолистное накрытие сферы Римана $L_{Q}^{0}$, разветвленное в узлах решетки $\bigcup_{n, m \in \mathbb{Z}}(n+m \tau) / 2$. Точки $Q_{k} \in L_{Q}^{0}$ являются образами узлов этой решетки. Мы занумеровали полупериоды $\omega_{k}$ так, что

$$
\pi_{u Q} \omega_{k}=Q_{k}
$$

Поднятие функции $q_{k}: L_{Q}^{0} \rightarrow \overline{\mathbb{C}}$ на $\mathbb{C}_{u}-$ это двояко-периодическая функция с единственной особенностью - полюсом в $\omega_{3}$ (это прообраз точки $Q_{3}$ ) и нулем в $\omega_{k}, k \in\{0,1,2\}$. Полюс в $\omega_{3}$ и нуль в $\omega_{k}$ - двойные, потому что $\pi_{T Q}$, а следовательно, и $\pi_{u Q}$ имеет ветвление второго порядка над $Q_{k} \in L_{Q}^{0}$. Эллиптическая 
инволюция $u \rightarrow-u$, переставляющая листы накрытия $\pi_{T Q}$, тривиально действует на $L_{Q}^{0}$, следовательно, для любого $k \in\{0,1,2\} \pi_{u Q}^{*} q_{k}: \mathbb{C}_{u} \rightarrow \overline{\mathbb{C}}-$ четная функция переменной $u$.

Поскольку значения функции $q_{1}$ во всех точках $Q_{j}$ известны, то имеющейся информации достаточно для построения $\pi_{u Q}^{*} q_{1}$ с помощью стандартных функций на $\mathbb{C}_{u}$ (эллиптических и тэта-функций).

УтВЕРЖДЕНИЕ 18. Имеет место представление

$$
\begin{aligned}
\pi_{u Q}^{*} q_{1} & =-\left(\frac{\theta_{0}((1+\tau) / 2) \theta_{2}(u)}{\theta_{2}((1+\tau) / 2) \theta_{0}(u)}\right)^{2} \\
& =\frac{\theta_{2}^{2} \theta_{2}^{2}(u)}{\theta_{0}^{2} \theta_{0}^{2}(u)}=\frac{k^{2}}{k^{\prime 2}} \operatorname{cn}^{2}(v, k)=-\frac{1}{e_{1}-e_{2}}\left(\wp\left(u-\omega_{3}\right)-e_{2}\right),
\end{aligned}
$$

где

$$
\begin{gathered}
v=2 K u, \quad 2 K:=\pi \theta_{3}^{2}, \quad k=\frac{\theta_{2}^{2}}{\theta_{3}^{2}}, \quad k^{\prime}=\frac{\theta_{0}^{2}}{\theta_{3}^{2}}, \\
e_{k}:=\wp\left(\omega_{k}\right), \quad \theta_{k}:=\theta_{k}(0 \mid \tau), \quad \tau=\frac{\omega_{3}}{\omega_{1}}, \quad \omega_{1}=\frac{1}{2} .
\end{gathered}
$$

ДокАЗАтЕЛьСтво. Эта и только эта функция с периодами $2 \omega_{1}=1,2 \omega_{3}=\tau$, четная по $u$, имеет нуль в $\omega_{1}$, полюс в $\omega_{3}$ и значение -1 в $\omega_{2}=-(1+\tau) / 2$.

Далее не будем писать символ $\pi_{u Q}^{*}$ перед $q_{1}$, подразумевая его: $q_{1}=q_{1}(u, \tau)$.

Сравнивая значения в полупериодах функции $q_{1}$, выраженной через тэтафункции и в обозначениях Якоби и Вейерштрасса, получим

$$
t=-q_{1}\left(Q_{0}\right)=-\frac{k^{2}}{k^{\prime 2}} \mathrm{cn}^{2}(0)=-\frac{\theta_{2}^{4}}{\theta_{0}^{4}}=-\frac{e_{3}-e_{2}}{e_{1}-e_{2}}=\frac{k^{2}}{k^{2}-1} .
$$

В точке $Q_{3}$ функция $q_{1}$ имеет полюс. Сравним асимптотики. Положим $v=$ $v_{1}+i K^{\prime}, v_{1} \rightarrow 0:$

$$
\begin{gathered}
q_{1}=\frac{k^{2}}{k^{\prime 2}} \operatorname{cn}^{2}(v, k)=\frac{k^{2}}{k^{\prime 2}} \operatorname{cn}^{2}\left(v_{1}+i K^{\prime}\right)=-\frac{1}{k^{\prime 2}} \frac{\mathrm{dn}^{2}\left(v_{1}\right)}{\operatorname{sn}^{2}\left(v_{1}\right)}=-\frac{1}{k^{\prime 2} v_{1}^{2}}\left(1+\mathbf{O}\left(v_{1}^{2}\right)\right), \\
\wp\left(u_{1}\right)-e_{2}=\frac{1}{u_{1}^{2}}\left(1+\mathbf{O}\left(u_{1}\right)\right), \quad u_{1} \rightarrow 0, \quad u_{1}:=u-\omega_{3}=\frac{v_{1}}{2 K} .
\end{gathered}
$$

Из этого следует, что

$$
e_{1}-e_{2}=4 K^{2} k^{\prime 2}=\pi^{2} \frac{\theta_{3}^{4} \theta_{0}^{4}}{\theta_{3}^{4}}=\pi^{2} \theta_{0}^{4} .
$$

Выразим оставшиеся функции $q_{2}$ и $q_{0}$ :

$$
\begin{aligned}
& q_{2}=-\left(1+q_{1}\right)=-\left(1+\frac{k^{2}}{k^{\prime 2}} \mathrm{cn}^{2} v\right)=\frac{-1}{k^{\prime 2}} \operatorname{dn}^{2} v \\
& q_{0}=-\left(t+q_{1}\right)=-\left(-\frac{k^{2}}{k^{\prime 2}}+\frac{k^{2}}{k^{\prime 2}} \mathrm{cn}^{2} v\right)=\frac{k^{2}}{k^{\prime 2}} \operatorname{sn}^{2} v .
\end{aligned}
$$


Отсюда получаем

$$
\mathscr{P}_{3}=q_{1} q_{2} q_{0}=-\frac{k^{4}}{k^{\prime 6}} \operatorname{sn}^{2} v \mathrm{cn}^{2} v \mathrm{dn}^{2} v
$$

Выберем знак корня:

$$
\sqrt{\mathscr{P}_{3}}=+i \frac{k^{2}}{k^{\prime 3}} \operatorname{sn} v \operatorname{cn} v \operatorname{dn} v .
$$

Поскольку (при постоянном $\tau$ )

$$
d q_{1}=-2 \frac{k^{2}}{k^{\prime 2}} \operatorname{cn} v \operatorname{sn} v \operatorname{dn} v d v, \quad v\left(Q_{0}\right)=0, \quad v\left(Q_{1}\right)=2 K=\pi \theta_{3}^{2},
$$

то

$$
\int_{q_{1}\left(Q_{0}\right)}^{q_{1}\left(Q_{1}\right)} \frac{d q_{1}}{\sqrt{\mathscr{P}_{3}}}=-\frac{2 k^{2} k^{\prime 3}}{i k^{\prime 2} k^{2}} \int_{0}^{2 K} d v=2 i k^{\prime} \cdot 2 K=2 \pi i \theta_{0}^{2} .
$$

Подставив значение $d \tau_{1-2} / d \tau$ в формулу (70), где вместо $\chi_{k}^{*} q_{1}$ поставлена сдвинутая на полупериод $\omega_{k}$ функция $q_{1}$ (формула $(81)$ ), получим

$$
2 \pi i F \frac{d \tau_{1-2}}{d \tau}=\sum_{j=1}^{3} a_{j j} \wp\left(u-\omega_{j}\right)+\left(a_{00}-\sqrt{2 a_{00}}\right) \wp(u) .
$$

Итак, уравнение Пенлеве VI принимает вид

$$
(2 \pi i)^{2} \frac{d^{2} u}{d \tau^{2}}=\sum_{j=1}^{3} a_{j j \wp}\left(u-\omega_{j}\right)+\left(a_{00}-\sqrt{2 a_{00}}\right) \wp(u)+\tilde{\phi},
$$

где

$$
\tilde{\phi}:=d \phi \wedge d u+d \phi^{2} \wedge d \tau /(4 \pi i)
$$

(см. формулу (78)). Заметим, что этот член возник из-за явной зависимости от $\tau$ замены $q_{1} \rightarrow u$ (формула (76)). Последующую выкладку можно рассматривать как иллюстрацию сложности "мира модулярных функций” по сравнению с "миром рациональных функций" - аналогичное вычисление, связанное с явной зависимостью замены от $t$, но в рациональных функциях, умещается в одну строчку:

$$
0=d \log q_{t} \wedge d q_{t} \quad \Longrightarrow \quad \frac{d q_{t}}{q_{t}} \wedge d q_{1}+\frac{d q_{t}}{q_{t}} \wedge d t=0 .
$$

ТЕорема 14. Нормированный на единицу (т.е. $\oint d u=1)$ абелев интеграл $(1-$ го рода) $u \in \mathbb{C}$ зависит от модуля тора $\tau$ следующим образом:

$$
\phi:=-\left.2 \pi i \frac{\partial}{\partial \tau}\right|_{q_{1}} u=\log ^{\prime} \theta_{1}(u \mid \tau) .
$$

ДокАЗАТЕЛЬСТВо. При постоянном значении $q_{1}$

$$
0=d \log q_{1}=\left.\left.\frac{\partial}{\partial u}\right|_{\tau} \log q_{1} d u\right|_{q_{1}}+\left.\left.\frac{\partial}{\partial \tau}\right|_{u} \log q_{1} d \tau\right|_{q_{1}},
$$


следовательно,

$$
\phi=\left.2 \pi i \frac{\partial}{\partial \tau}\right|_{u} \log q_{1}\left(\left.\frac{\partial}{\partial u}\right|_{\tau} \log q_{1}\right)^{-1} .
$$

Функция $q_{1}$ имеет представление (81):

$$
q_{1}=\frac{\theta_{2}^{2}}{\theta_{0}^{2}} \frac{\theta_{2}^{2}(u)}{\theta_{0}^{2}(u)}=\frac{k^{2}}{k^{\prime 2}} \mathrm{cn}^{2}(v, k)
$$

так что доказательство теоремы сводится к проверке тождества, содержащего тэта-функции. Применим (79). Учитывая, что $\theta_{0}^{\prime}=\theta_{2}^{\prime}=0$ и

$$
\left.\frac{d}{d u}\right|_{\tau} \log q_{1}=2 \log ^{\prime} \frac{\theta_{2}(u)}{\theta_{0}(u)}
$$

получим

$$
2 \phi=4 \pi i \frac{\frac{\partial}{\partial \tau} \log \frac{\theta_{2} \theta_{2}(u)}{\theta_{0} \theta_{0}(u)}}{\frac{\partial}{\partial u} \log \frac{\theta_{2} \theta_{2}(u)}{\theta_{0} \theta_{0}(u)}}=\frac{\log ^{\prime \prime} \frac{\theta_{2}}{\theta_{0}}}{\log ^{\prime} \frac{\theta_{2}(u)}{\theta_{0}(u)}}+\log ^{\prime} \log ^{\prime} \frac{\theta_{2}(u)}{\theta_{0}(u)}+\log ^{\prime} \theta_{2}(u) \theta_{0}(u)
$$

и, поскольку $\log ^{\prime} \log ^{\prime} \frac{\theta_{2}(u)}{\theta_{0}(u)}=\log ^{\prime} \frac{\theta_{1}(u) \theta_{3}(u)}{\theta_{0}(u) \theta_{2}(u)}$,

$$
2 \phi=2 \log ^{\prime} \theta_{1}(u)+\frac{\log ^{\prime \prime} \frac{\theta_{2}}{\theta_{0}}+\left(\log ^{\prime} \frac{\theta_{2}(u)}{\theta_{0}(u)}\right)\left(\log ^{\prime} \frac{\theta_{3}(u)}{\theta_{1}(u)}\right)}{\log ^{\prime} \frac{\theta_{2}(u)}{\theta_{0}(u)}} .
$$

Покажем, что второе слагаемое равно нулю. Функции

$$
\log ^{\prime} \frac{\theta_{2}(u)}{\theta_{0}(u)} \quad \text { и } \quad \log ^{\prime} \frac{\theta_{3}(u)}{\theta_{1}(u)}
$$

имеют полюсы и нули в полупериодах тора, причем там, где у одной из них нуль, у другой - полюс, так что их произведение от $u$ не зависит - функция только $\tau$. Вычислим эту функцию

$$
\begin{aligned}
\log ^{\prime} \frac{\theta_{2}(u)}{\theta_{0}(u)} \log ^{\prime} \frac{\theta_{3}(u)}{\theta_{1}(u)} & =-(2 K)^{2}\left(\frac{d}{d v} \log \operatorname{cn} v\right) \frac{d}{d v} \log \operatorname{cn}(v+K) \\
& =-4 K^{2} \frac{\operatorname{sn} v \operatorname{dn} v}{\operatorname{cn} v} \frac{\operatorname{sn}(v+K) \operatorname{dn}(v+K)}{\operatorname{cn}(v+K)}=4 K^{2}
\end{aligned}
$$

в то же время

$$
\log ^{\prime \prime} \frac{\theta_{2}}{\theta_{0}}=\left.(2 K)^{2} \frac{d^{2}}{d v^{2}} \log \operatorname{cn} v\right|_{v=0}=-\left.4 K^{2} \frac{d}{d v} \frac{\operatorname{sn} v \operatorname{dn} v}{\operatorname{cn} v}\right|_{v=0}=-4 K^{2}
$$

теорема доказана. 
Продифференцируем (79) по $u$. Поскольку $\phi=\log ^{\prime} \theta_{1}(u)$, получим

$$
d \phi \wedge d u+d \phi^{2} \wedge \frac{d \tau}{4 \pi i}=-d \log ^{\prime \prime} \theta_{1}(u) \wedge \frac{d \tau}{4 \pi i} .
$$

Именно при таком выборе переменных $u$ и $\tau$ разность

$$
4 \pi i \frac{\partial \phi}{\partial \tau}-\frac{\partial \phi^{2}}{\partial u}
$$

оказалась эллиптической функцией $-4 \pi i \log ^{\prime \prime} \theta_{1}(u)$. Так как $\log ^{\prime \prime} \theta_{1}(u)$ и $-\wp(u)$ имеют одинаковые периоды и особенности, то они отличаются на константу,

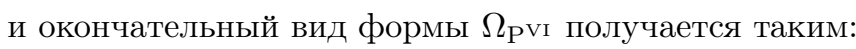

$$
\begin{aligned}
\Omega_{\mathrm{PVI}} & =d p \wedge d u-d\left(\frac{p^{2}}{2}-\mathscr{F}(u, \tau)\right) \wedge \frac{d \tau}{2 \pi i}, \\
\mathscr{F}(u, \tau)= & \sum_{j=1}^{3} a_{j j} \wp\left(u-\omega_{j}\right)+\left(\sqrt{a_{00}}-\frac{1}{\sqrt{2}}\right)^{2} \wp(u), \\
p= & i P \frac{\theta_{3}^{2} \theta_{2}^{2}}{\theta_{0}^{4}} \frac{\theta_{1}(u) \theta_{2}(u) \theta_{3}(u)}{\theta_{0}^{3}(u)} 2 \pi i \theta_{0}^{2}-\log ^{\prime} \theta_{1}(u) \\
= & -2 \pi P \frac{\theta_{3}^{2} \theta_{2}^{2}}{\theta_{0}^{2}} \frac{\theta_{1}(u) \theta_{2}(u) \theta_{3}(u)}{\theta_{0}^{3}(u)}-\log ^{\prime} \theta_{1}(u) \\
= & -\left(p_{1}+\frac{\sqrt{a_{11} / 2}}{q_{1}}-\frac{\sqrt{a_{22} / 2}}{q_{2}}-\frac{\sqrt{a_{00} / 2}}{q_{t}}\right) \\
u= & \frac{1}{2 \pi i \theta_{0}^{2}} \int_{-t}^{q_{1}} \frac{\theta_{3}^{2} \theta_{2}^{2}}{\theta_{1}^{2}} \frac{\theta_{1}(u) \theta_{2}(u) \theta_{3}(u)}{\theta_{0}^{3}(u)}-\log ^{\prime} \theta_{1}(u), \\
& \times 2 q+1)(q+1)
\end{aligned}
$$

Уравнение Пенлеве VI принимает вид

$$
(2 \pi i)^{2} \frac{d^{2}}{d \tau^{2}} u=\sum_{j=1}^{3} a_{j j} \wp^{\prime}\left(u-\omega_{j}\right)+\left(\sqrt{a_{00}}-\frac{1}{\sqrt{2}}\right)^{2} \wp^{\prime}(u),
$$

где функция $\wp^{\prime}$ строится по решетке периодов $2 \omega_{1}=1,2 \omega_{3}=\tau$.

\section{7. Поверхность начальных данных $\mathrm{P}^{\mathrm{VI}}$ (поверхность Окамото) и определяющее многообразие}

7.1. Симплектическая склейка квадрик - связь между координатами, построенными с помощью разных сопутствующих базисов. В п. 5.3 многообразие тетраэдров $M_{a_{\overline{i i}}}$ было покрыто тремя (см. формулу (59)) областями-окрестностями $U(.$.$) , каждая из которых симплектически изоморфна$ аффинной квадрике $\mathscr{C}_{a_{i i}}, i \in\{1,2,3\}$. Эквивалентной, правда менее симметричной, является следующая переформулировка процедуры построения $M_{a_{\bar{i}}}$. 
Рассмотрим одну из квадрик, например $\mathscr{C}_{a_{11}}$, и "приклеим" к ней остальные $\mathscr{C}_{a_{22}}$ и $\mathscr{C}_{a_{33}}$, т. е. отождествим некоторые точки $\mathscr{C}_{a_{11}}$ и $\mathscr{C}_{a_{i i}}, i=2,3$. Выделим в $\mathscr{C}_{a_{11}}$ и $\mathscr{C}_{a_{i i}}$ открытые множества (окрестности) $V_{1, i} \subset \mathscr{C}_{a_{11}}$ и $V_{i, 1} \subset \mathscr{C}_{a_{i i}}$ и зададим симплектические изоморфизмы

$$
\psi_{1 i}: V_{1, i} \stackrel{\sim}{\rightarrow} V_{i, 1}, \quad i=2,3 .
$$

Это позволит определить $M_{a_{\bar{i}}}$ как абстрактное многообразие - симплектические карты $\phi_{i}$ и $\phi_{i}^{*}$ квадрики $\mathscr{C}_{a_{i i}}$ (см. определение 2 на с. 80) в композиции с $\psi_{1 i}$ дадут функции переклейки многообразия $M_{a_{i i}}$ :

$$
\mathbb{C}_{p_{1} q_{1}}^{2} \supset \phi_{1}^{-1}\left(V_{1, i} \cap U\right) \stackrel{\phi_{1}}{\longrightarrow} V_{1, i} \cap U \stackrel{\psi_{1 i}}{\longrightarrow} V_{i 1} \cap U \stackrel{\phi_{i}^{-1}}{\longrightarrow} \mathbb{C}_{p_{i} q_{i}}^{2} .
$$

Эта схема показывает, как действует функция переклейки $\phi_{i}^{-1} \circ \psi_{1 i} \circ \phi_{1}$, отображающая друг в друга некоторые области в $\mathbb{C}^{2}$. Аналогичным образом строятся и остальные:

$$
\left(\phi_{i}^{*}\right)^{-1} \circ \psi_{1 i} \circ \phi_{1}, \quad \phi_{i}^{-1} \circ \psi_{1 i} \circ \phi_{1}^{*}, \quad\left(\phi_{i}^{*}\right)^{-1} \circ \psi_{1 i} \circ \phi_{1}^{*} .
$$

Заметим, что мы работаем с алгебраическими окрестностями, т. е. "очень большими" - дополнение в $M_{a_{\bar{i}}}$ к любой из областей $U\left(-\sqrt{2 a_{00}} ; \sqrt{2 a_{i i}}, j\right), i, j \in$ $\{1,2,3\}$, менъшей размерности, чем размерность самого $M_{a_{\bar{i}}}$. Любая такая область покрывает всю поверхность $M_{a_{\bar{i}}}$ за исключением нескольких линий на ней.

Приведенные в предыдущем разделе формулы явным образом устанавливают изоморфизм между $U\left(-\sqrt{2 a_{00}} ; \sqrt{2 a_{22}}, 3\right)$ и $\mathscr{C}_{a_{11}}$, т. е. всем $M_{a_{\bar{i}}}$ за вычетом трех линий: на точках (наборах $A^{(\bar{i})}$ ) одной из них $\sigma_{-}=\sigma^{\left(-\sqrt{2 a_{00}}\right.}$ ) является для $\operatorname{ad}_{A^{(2)}}$ собственным вектором с собственным числом $\sqrt{2 a_{22}}$, а две остальные - это компоненты $Q_{-\sqrt{2 a_{00}}}^{-1}\left(\left\langle A^{(3)}, \sigma^{\left(-\sqrt{2 a_{00}}\right)}\right\rangle=0\right)$.

Развиваемая сейчас точка зрения (на построение $M_{a_{\overline{i i}}}$ ) состоит в том, что мы с помощью отображений $\psi_{1,2}$ и $\psi_{1,3}$, приклеиваем к $\mathscr{C}_{a_{11}}$ эти линии (дивизоры).

Нам это нужно для того, чтобы аналогичным образом приклеить (теперь уже ко всему $M_{a_{\bar{i}}}$ ) еще один дивизор, назовем его $\mathscr{D}^{t}$, точкам которого уже не будут соответствовать никакие тетраэдры (наборы $A^{(\bar{i})}$, или уравнения вида (1)). Забегая вперед, скажем, что точкам этого дивизора будут соответствовать специальным образом уходящие на бесконечность наборы $A^{(\bar{i})}: A_{j k}^{(i)} \rightarrow \infty$. Словосочетание "специальным образом" тут означает, что на матричные коэффициенты $A_{j k}^{(i)}$ будут наложены некоторые зависящие от $t$ ограничения.

Эти ограничения (связи) будут такими, что через соответствующие координатные функции $(p(t), q(t)), p=p\left(A^{(\bar{i})}\right), q=q\left(A^{(\bar{i})}\right)$, сингулярные при $t=t_{0}$, решения $\left(p_{t_{0}}^{\text {sing }}(t), q_{t_{0}}^{\text {sing }}(t)\right)$ системы Пенлеве VI будут выражаться гладким образом. Другими словами, новая, "руками” построенная, область будет нужна для описания сингулярных при $t=t_{0}$ решений системы Пенлеве VI.

7.1.1. Склейка $\mathscr{C}_{R_{x}^{2}} \uplus_{C}^{Q} \mathscr{C}_{R_{y}^{2}}$. Выясним, как к $\mathscr{C}_{a_{11}}$ приклеивается $\mathscr{C}_{a_{33}}$, параметризующая слой $\left\langle A^{(3)}, \sigma^{-\sqrt{2 a_{00}}}\right\rangle=0$ отображения $Q_{-\sqrt{2 a_{00}}}$, где $q_{1}$ и $q_{2}$ имеют полюсы. 
Пусть $X_{i}$ - координаты на $\mathscr{C}_{R_{x}^{2}}, Y_{i}-$ координаты на $\mathscr{C}_{R_{y}^{2}}$ :

$$
\mathscr{C}_{R_{x}^{2}}: \quad X_{1} X_{2}+X_{3}^{2}=\frac{R_{x}^{2}}{2}, \quad \mathscr{C}_{R_{y}^{2}}: \quad Y_{1} Y_{2}+Y_{3}^{2}=\frac{R_{y}^{2}}{2}
$$

Если, скажем, $R_{x}^{2} \neq 0$, то задание $\left(X_{1}, X_{2}, X_{3}\right)$ эквивалентно заданию точки $\mathscr{C}_{R_{x}^{2}}$; если же $R_{x}^{2}=0$, то при $X_{1}=X_{2}=X_{3}=0$ нужно еще задать $x_{1}$ : $x_{2}: x_{3} \in \mathbb{C P}^{2}$ с коники $x_{1} x_{2}+x_{3}^{2}=0$. Отождествим точку $\left(X_{1}, X_{2}, X_{3}\right) \in \mathscr{C}_{R_{x}^{2}}$ с точкой $\left(Y_{1}, Y_{2}, Y_{3}\right) \in \mathscr{C}_{R_{y}^{2}}$, если

$$
\begin{aligned}
& X_{1}=\frac{1}{Y_{1}}, \\
& X_{3}=-Y_{3}-C, \quad C \in \mathbb{C}, \\
& X_{2}=\frac{R_{x}^{2} / 2-X_{3}^{2}}{X_{1}}=Y_{1}\left(\frac{R_{x}^{2}}{2}-\left(Y_{3}+C\right)^{2}\right),
\end{aligned}
$$

т. е. одной точке будущего многообразия $\mathscr{C}_{R_{x}^{2}} \uplus_{C}^{Q} \mathscr{C}_{R_{y}^{2}}$ будут соответствовать точки $\mathscr{C}_{R_{x}^{2}}$ и $\mathscr{C}_{R_{y}^{2}}$, связанные (87).

ЗАмЕчАНИЕ 23. Преобразование (87) бирационально и инволютивно - обратное получится заменой $X \rightleftarrows Y$.

В канонических переменных $(p, q)$ и $\left(p^{*}, q^{*}\right)$ склейка (87) записывается так:

$$
q_{x}=\frac{1}{q_{y}}, \quad p_{x}=-q_{y}\left(p_{y} q_{y}+\sqrt{\frac{R_{x}^{2}}{2}}+\sqrt{\frac{R_{y}^{2}}{2}}+C\right) .
$$

Мы снабдили $p$ и $q$ соответствующим квадрике индексом. Последнее равенство - следствие того, что

$$
\begin{aligned}
X_{3} & =p_{x} q_{x}+\sqrt{\frac{R_{x}^{2}}{2}}=-\left(p_{x}^{*} q_{x}^{*}+\sqrt{\frac{R_{x}^{2}}{2}}\right) \\
& =-\left(Y_{3}+C\right)=-\left(p_{y} q_{y}+\sqrt{\frac{R_{y}^{2}}{2}}+C\right)=p_{y}^{*} q_{y}^{*}+\sqrt{\frac{R_{y}^{2}}{2}}-C .
\end{aligned}
$$

Очевидно, что $d p_{x} \wedge d q_{x}=d p_{y} \wedge d q_{y}$ - склейка симплектическая. Многообразие $\mathscr{C}_{R_{x}^{2}} \uplus_{C}^{Q} \mathscr{C}_{R_{y}^{2}}$ можно трактовать как одну из квадрик, например $\mathscr{C}_{R_{x}^{2}}$, с двумя приклеенными на бесконечности по $X_{1}$ линиями (простыми дивизорами):

1) если $R_{y} \neq 0$, то это $Y_{1}=0, Y_{3}= \pm \sqrt{R_{y}^{2} / 2}, Y_{2} \in \mathbb{C}$;

2) если $R_{y}=0$, то это $Y_{1}=Y_{3}=0, Y_{2} \in \mathbb{C}$ и

$$
Y_{1}=Y_{2}=Y_{3}=0, \quad y_{1}: y_{2}: y_{3}=1:-p^{2}: p=\left(p^{*}\right)^{2}:-1: p^{*}
$$


$\mathrm{K}$ точке $Y_{1}=0, Y_{3}= \pm \sqrt{R_{y}^{2} / 2}, Y_{2}=$ const $\neq 0$ добавленного дивизора стремится точка $\left(X_{1}(S), X_{2}(S), X_{3}(S)\right), S \rightarrow 0$, квадрики $\mathscr{C}_{R_{x}^{2}}$ такая, что

$$
\begin{aligned}
& X_{1}(S)=\frac{1}{S} \frac{Y_{2}}{2 \sqrt{R_{y}^{2} / 2}-S} \rightarrow \infty, \\
& X_{2}(S)=\frac{S}{Y_{2}}\left(2 \sqrt{\frac{R_{y}^{2}}{2}}-S\right)\left(\frac{R_{x}^{2}}{2}-\left(\sqrt{\frac{R_{y}^{2}}{2}}-S \pm C\right)^{2}\right) \rightarrow 0, \\
& X_{3}(S)=\mp\left(\sqrt{\frac{R_{y}^{2}}{2}}-S \pm C\right) \rightarrow \mp \sqrt{\frac{R_{y}^{2}}{2}} .
\end{aligned}
$$

Именно такой получится зависимость $X_{i}(S)$, если положить

$$
Y_{3}= \pm\left(\sqrt{\frac{R_{x}^{2}}{2}}-S\right)
$$

В этом случае

и, значит,

$$
0=Y_{1} Y_{2}+Y_{3}^{2}-\frac{R_{y}^{2}}{2}=Y_{1} Y_{2}-2 \sqrt{\frac{R_{y}^{2}}{2}} S+S^{2}
$$

$$
Y_{1}=\frac{S}{Y_{2}}\left(2 \sqrt{\frac{R_{y}^{2}}{2}}-S\right) \rightarrow 0 .
$$

Если же $R_{y}=0$ и линии, соответствующие $+\sqrt{R_{y}^{2} / 2}$ и $-\sqrt{R_{y}^{2} / 2}$, совпадают, то к точке $Y_{1}=Y_{2}=Y_{3}=0, y_{1}: y_{2}: y_{3}=1:-p^{2}: p$, второго добавленного дивизора (раздутой вершины конуса) стремится $\left(X_{1}(S), X_{2}(S), X_{3}(S)\right)$ :

$$
\begin{aligned}
& X_{1}(S)=\frac{1}{S} \rightarrow \infty, \\
& X_{2}(S)=S\left(\frac{R_{x}^{2}}{2}-(p S+C)^{2}\right) \rightarrow 0, \\
& X_{3}(S)=-p S-C \rightarrow-C .
\end{aligned}
$$

ЗАмЕЧАНИЕ 24. Имеется естественное отображение

$$
\mathscr{C}_{R_{x}^{2}} \uplus_{C}^{Q} \mathscr{C}_{R_{y}^{2}} \rightarrow \mathbb{C} \mathbf{P}^{1}:\left(X_{1}, X_{2}, X_{3}\right) \rightarrow\left(X_{1}: 1\right)=\left(1: Y_{1}\right) \longleftarrow\left(Y_{1}, Y_{2}, Y_{3}\right)
$$

Общим слоем его являются параболы, лежащие на $\mathscr{C}_{R_{x}^{2}}$ и $\mathscr{C}_{R_{y}^{2}}$ :

$$
\begin{array}{cl}
\bigcup_{X_{2} \in \mathbb{C}} X_{1} X_{2}+X_{3}^{2} & =\frac{R_{x}^{2}}{2}, \quad X_{1}=\text { const } \in \mathbb{C}, \\
\bigcup_{Y_{2} \in \mathbb{C}} Y_{1} Y_{2}+Y_{3}^{2} & =\frac{R_{y}^{2}}{2}, \quad Y_{1}=\text { const } \in \mathbb{C} .
\end{array}
$$

На особенных слоях $X_{3}^{2}=R_{x}^{2} / 2$ и $Y_{3}^{2}=R_{y}^{2} / 2$ параболы вырождаются в пары прямых:

$$
\begin{array}{lll}
X_{1}=0, & X_{3}= \pm \sqrt{\frac{R_{x}^{2}}{2}}, & X_{2} \in \mathbb{C}, \\
Y_{1}=0, & Y_{3}= \pm \sqrt{\frac{R_{y}^{2}}{2}}, & Y_{2} \in \mathbb{C} .
\end{array}
$$


7.1.2. Склейка $\mathscr{C}_{R_{x}^{2}} \uplus_{t}^{T} \mathscr{C}_{R_{y}^{2}}$. Пусть $t \in \mathbb{C}-$ некоторый параметр. Очевидно, что если склеить точки квадрик (86), сдвинув их $q$-координаты на постоянную величину " $t$ ", а $p$-координаты оставить неизменными: $q_{x}=q_{y}+t, p_{x}=p_{y}$, то такая склейка будет симплектической: $d p_{x} \wedge d q_{x}=d p_{y} \wedge d q_{y}$. Из соображений симметрии, однако, нам будет удобнее перенести все переменные в одну часть равенства (поменяв знаки), что, конечно же, не изменит сути происходящего. Отождествим точки $\mathscr{C}_{R_{x}^{2}}$ и $\mathscr{C}_{R_{y}^{2}}$, чьи координаты удовлетворяют равенствам

$$
X_{1}+Y_{1}+t=0, \quad \frac{X_{3}-\sqrt{R_{x}^{2} / 2}}{X_{1}}+\frac{Y_{3}-\sqrt{R_{y}^{2} / 2}}{Y_{1}}=0,
$$

что эквивалентно

$$
\begin{aligned}
& X_{3}=p_{x} X_{1}+\sqrt{\frac{R_{x}^{2}}{2}}=-p_{y} X_{1}+\sqrt{\frac{R_{x}^{2}}{2}}=p_{y}\left(Y_{1}+t\right)+\sqrt{\frac{R_{x}^{2}}{2}}, \\
& X_{2}=-p_{x}\left(X_{3}+\sqrt{\frac{R_{x}^{2}}{2}}\right)=p_{y}\left(p_{y}\left(Y_{1}+t\right)+2 \sqrt{\frac{R_{x}^{2}}{2}}\right) .
\end{aligned}
$$

Получаем закон склейки

$$
p_{x}+p_{y}=0, \quad q_{x}+q_{y}+t=0
$$

или

$$
\begin{aligned}
& X_{1}=-\left(Y_{1}+t\right), \\
& X_{2}=\frac{Y_{3}-\sqrt{R_{y}^{2} / 2}}{Y_{1}}\left(\frac{Y_{3}-\sqrt{R_{y}^{2} / 2}}{Y_{1}}\left(Y_{1}+t\right)+2 \sqrt{\frac{R_{x}^{2}}{2}}\right), \\
& X_{3}=\frac{Y_{3}-\sqrt{R_{y}^{2} / 2}}{Y_{1}}\left(Y_{1}+t\right)+\sqrt{\frac{R_{x}^{2}}{2}} .
\end{aligned}
$$

Это преобразование инволютивно - обратное получится заменой букв $X \rightleftarrows Y$.

Отметим, что линии, получившиеся из раздутых вершин, если таковые существуют, склеиваются регулярным образом (биективно), потому что в окрестностях таких линий координатами являются $(p, q)$-координаты, а они преобразуются регулярно (биективно):

$$
q_{x}=-q_{y}-t, \quad p_{x}=-p_{y}
$$

Геометрически склейка (90) означает, что сечения обеих квадрик, т. е. параболы

$$
\begin{array}{ll}
X_{2} \operatorname{const}_{x}+X_{3}^{2}=\frac{R_{x}^{2}}{2}, & X_{1}=\operatorname{const}_{x} \\
Y_{2} \operatorname{const}_{y}+Y_{3}^{2}=\frac{R_{y}^{2}}{2}, & Y_{1}=\operatorname{const}_{y}=-\operatorname{const}_{x}-t
\end{array}
$$

отождествляются между собой. Там, где $X_{1} Y_{1} \neq 0$, многообразие $\mathscr{C}_{R_{x}^{2}} \uplus_{t}^{T} \mathscr{C}_{R_{y}^{2}}$ покрыто обеими квадриками. Над значением $X_{1}=0$ "висят" особенные слои это $X_{3}= \pm \sqrt{R_{x}^{2} / 2}, X_{2} \in \mathbb{C}^{2}$, либо $X_{3}=0, X_{2} \in \mathbb{C}$ и $X_{1}=X_{2}=X_{3}=0$, 
$x_{1}: x_{2}: x_{3}=1:-p^{2}: p, x_{1}: x_{3} \in \mathbb{C P}^{1}$. Одна из этих линий "приклеена" к $\mathscr{C}_{R_{y}^{2}}-$ это $X_{3}=\sqrt{R_{x}^{2} / 2} \neq 0$ :

$$
\begin{aligned}
Y_{1} & =-t, \\
Y_{2} & =\frac{-X_{2}}{X_{3}+\sqrt{R_{x}^{2} / 2}}\left(\frac{-X_{2}}{X_{3}+\sqrt{R_{x}^{2} / 2}}\left(X_{1}+t\right)+2 \sqrt{\frac{R_{y}^{2}}{2}}\right) \\
& =\frac{-X_{2}}{2 \sqrt{R_{x}^{2} / 2}}\left(\frac{-X_{2}}{2 \sqrt{R_{x}^{2} / 2}} t+2 \sqrt{\frac{R_{y}^{2}}{2}}\right), \\
Y_{3} & =\frac{-X_{2}}{X_{3}+\sqrt{R_{x}^{2} / 2}}\left(X_{1}+t\right)+\sqrt{\frac{R_{x}^{2}}{2}}=\frac{-X_{2}}{2 \sqrt{R_{x}^{2} / 2}} t+\sqrt{\frac{R_{x}^{2}}{2}},
\end{aligned}
$$

либо (если $\left.R_{x}=0\right)$ - раздутая вершина конуса:

$$
\begin{aligned}
& Y_{1}=-t-X_{1}, \\
& Y_{2}=p\left(p\left(X_{1}+t\right)+2 \sqrt{\frac{R_{y}^{2}}{2}}\right)=p\left(p t+2 \sqrt{\frac{R_{y}^{2}}{2}}\right), \\
& Y_{3}=p\left(X_{1}+t\right)=p t,
\end{aligned}
$$

а другая $\left(X_{3}=-\sqrt{R_{x}^{2} / 2}, X_{1}=0, X_{2} \in \mathbb{C}\right)$ "не приклеена" - покрыта только одной квадрикой $\mathscr{C}_{R_{x}^{2}}$.

Подытожим.

$\mathrm{y} \mathscr{C}_{R_{x}^{2}} \uplus_{t}^{T} \mathscr{C}_{R_{y}^{2}}$ над точкой $X_{1}=0$ всегда находится один раздутый дивизор прямая, параметризованная значениями $X_{2} \in \mathbb{C}$, или $\mathbb{C P}^{1}$, параметризованная $x_{1}: x_{3}$.

Ее точки не принадлежат $\mathscr{C}_{R_{y}^{2}}$, потому что на ней

$$
\begin{aligned}
Y_{2} & =\frac{-X_{2}}{X_{3}+\sqrt{R_{x}^{2} / 2}}\left(\frac{-X_{2}}{X_{3}+\sqrt{R_{x}^{2} / 2}}\left(X_{1}+t\right)+2 \sqrt{\frac{R_{x}^{2}}{2}}\right) \\
& \sim t\left(\frac{X_{2}}{X_{3}+\sqrt{R_{x}^{2} / 2}}\right)^{2} \rightarrow \infty
\end{aligned}
$$

для всех $X_{2} \neq 0, X_{3} \mapsto-\sqrt{R_{x}^{2} / 2}$ независимо от значений $R_{x}, R_{y}$.

Аналогично и над $Y_{1}=0$ всегда существует прямая (иногда - проективная), добавленная к $\mathscr{C}_{R_{x}^{2}}$.

7.2. Поверхность Окамото. Определяющее многообразие. Система Пенлеве VI задана как поле направлений в $M_{a_{\bar{i}}} \times \Lambda$. Искомое направление направление вырождения формы

$$
\begin{aligned}
\Omega_{\mathrm{PVI}}= & d p_{1} \wedge d q_{1}-d\left(q_{1} q_{2} q_{t} p_{1}^{2}+2 p_{1}\left(\sqrt{\frac{a_{11}}{2}} q_{2} q_{t}-\sqrt{\frac{a_{22}}{2}} q_{1} q_{t}-\sqrt{\frac{a_{00}}{2}} q_{1} q_{2}\right)\right. \\
& \left.+q_{t}\left(\frac{a_{33}}{2}-\left(\sqrt{\frac{a_{00}}{2}}+\sqrt{\frac{a_{11}}{2}}+\sqrt{\frac{a_{22}}{2}}\right)^{2}\right)\right) \wedge d \tau_{1-2} \\
:=d p_{1} & \wedge d q_{1}-d H_{1 / 3} \wedge d \tau_{1-2} .
\end{aligned}
$$


Это - определение (обозначение) гамильтониана $H_{1 / 3}$. Индекс " $1 / 3$ " символизирует, что $q$-координата в этом представлении (карте) равна нулю в $Q_{1}$ и бесконечности в $Q_{3}: q=q_{1}\left(Q_{1}\right)=0, q_{1}\left(Q_{3}\right)=\infty$.

Формула (91) задает векторное поле в той части $M_{a_{\bar{i}}}$, в которой $p_{1}$ и $q_{1}$ являются координатами.

В разделе 4 (определения 1 и 2) были введены бо́льшие координатные окрестности - аффинные квадрики $\mathscr{C}_{a_{i i}}, i=1,2,3$. В частности, были построены координаты

$$
X_{1}^{(i)}, X_{2}^{(i)}, X_{3}^{(i)}: \quad X_{1}^{(i)} X_{2}^{(i)}+\left(X_{3}^{(i)}\right)^{2}=\frac{a_{i i}}{2} .
$$

Здесь верхний индекс в скобках показывает, на какой именно квадрике это координаты; занумерованы они так, что $X_{1}^{(i)}=0$ там, где $q_{i}=0$. Положим $i=1$. Эти координаты покрывают прообраз окрестности точки $q_{1}=0$ отображения $Q_{-\sqrt{2 a_{00}}}: M_{a_{\bar{i}}} \rightarrow L_{Q}^{0} \sim \mathbb{C P}^{1}-$ все $M_{a_{\bar{i}}}$ кроме слоя $Q_{-\sqrt{2 a_{00}}}$ над $Q_{3}$ и одной из двух компонент слоя над $Q_{2}$. Запишем гамильтониан $H_{1 / 3}$ в координатах $X_{1}^{(1)}, X_{2}^{(1)}, X_{3}^{(1)}$. Несмотря на то, что $q_{1}, p_{1}$ выражаются через $X_{i}^{(1)}$ лишь рационально (имеется $X_{1}^{(1)}$ в знаменателе), гамильтониан $H_{1 / 3}\left(p_{1}, q_{1}\right)$ обязан быть многочленом от $X_{i}^{(1)}$ - это линейная комбинация функций $a_{i j}$, не имеющих особенностей на всем $M_{a_{\bar{i}}}$, в частности на $U\left(-\sqrt{2 a_{00}} ; \sqrt{2 a_{22}}, 3\right)$, на всей области определения $q_{1}, p_{1}$

$$
\begin{gathered}
q_{1}=X^{(1)}, \quad p_{1}=\left(X_{3}^{(1)}-\frac{1}{X_{1}^{(1)}} \sqrt{\frac{a_{11}}{2}}\right) \\
X_{3}^{(1)}=p_{1} q_{1}+\sqrt{\frac{a_{11}}{2}}, \quad X_{2}^{(1)}=-p_{1}\left(p_{1} q_{1}+2 \sqrt{\frac{a_{11}}{2}}\right), \\
H_{1 / 3}=q_{2} q_{t}\left(p_{1}\left(p_{1} q_{1}+2 \sqrt{\frac{a_{11}}{2}}\right)\right)-2 p_{1} q_{1}\left(\sqrt{\frac{a_{22}}{2}} q_{t}+\sqrt{\frac{a_{00}}{2}} q_{2}\right) \\
-\left(q_{1}+t\right)\left(\frac{a_{33}}{2}-\left(\sqrt{\frac{a_{00}}{2}}+\sqrt{\frac{a_{11}}{2}}+\sqrt{\frac{a_{22}}{2}}\right)^{2}\right) \\
\left.=-\left(X_{1}^{(1)}+1\right)\left(X_{1}^{(1)}+t\right) X_{2}^{(1)}\right)\left(\sqrt{\frac{a_{22}}{2}}\left(X_{1}^{(1)}+t\right)+\sqrt{\frac{a_{00}}{2}}\left(X_{1}^{(1)}+1\right)\right) \\
\left.+2\left(X_{3}^{(1)}-\sqrt{\frac{a_{11}}{2}}+\sqrt{\frac{a_{11}}{2}}+\sqrt{\frac{a_{22}}{2}}\right)^{2}\right) . \\
-\left(X_{1}^{(1)}+t\right)\left(\frac{a_{33}}{2}-\left(\sqrt{\frac{a_{00}}{2}}\right)\right.
\end{gathered}
$$

Обратим внимание на симметрию выражения (91) относительно замены индекса у переменной $q \ldots$. Сделаем замену $q_{1} \rightarrow q_{t}: q_{t}=-\left(q_{1}+t\right)$. Если ввести переменную $p_{t}:=-p_{1}$, канонически сопряженную $q_{t}$, т. е.

$$
d p_{1} \wedge d q_{1}=\left.\left(d p_{t} \wedge d q_{t}\right)\right|_{t=\text { const }},
$$

то форма $\Omega_{\mathrm{PVI}}$ сохранит вид

$$
\Omega_{\mathrm{PVI}}=d p_{t} \wedge d q_{t}-d\left(p_{t}^{2} \mathscr{P}_{3}+2 p_{t} \mathscr{P}_{2}+\mathscr{P}_{1}\right) \wedge d \tau_{1-2},
$$


где $\mathscr{P}_{i}$ - опять многочлен степени " $i$ " по $q_{t}$. Вследствие явной зависимости замены $q_{1} \rightarrow q_{t}$ от $t$ :

$$
q_{1}+q_{t}+t=0, \quad p_{1}+p_{t}=0
$$

к гамильтониану $H_{1 / 3}$ добавится нетривиальное слагаемое.

Зависимость замены от времени имеет специальный аддитивный вид, т. е. "старое $q "$ + "новое $q "$ + "функция времени" =0, поэтому возникающий в гамильтониане член будет линеен по импульсу - войдет в $\mathscr{P}_{2}$, действительно,

$$
d p_{1} \wedge d q_{1}=d p_{t} \wedge d\left(q_{t}+t\right)=d p_{t} \wedge d q_{t}-d\left(\frac{d t}{d \tau_{1-2}}\right) p_{t} \wedge d \tau_{1-2}
$$

Отметим, что именно этот член дает добавку к параметру $\delta$ уравнения Пенлеве VI (остальные три параметра это просто $a_{i i}$, см. с. 103). Вычислим $\mathscr{P}_{2}$. Поскольку $d t / d \tau_{1-2}=-t(t-1), q_{1}=q_{1}\left(Q_{t}\right)-q_{t}, q_{2}=q_{2}\left(Q_{t}\right)+q_{t}$ (так как $\left.q_{1}\left(Q_{t}\right)=-t, q_{2}\left(Q_{t}\right)=t-1\right)$ и

$$
q_{1} q_{2}=q_{1}\left(Q_{t}\right) q_{2}\left(Q_{t}\right)+q_{t}\left(q_{1}\left(Q_{t}\right)-q_{2}\left(Q_{t}\right)-q_{t}\right)=-t(t-1)-q_{t}\left(q_{t}+2 t-1\right),
$$

Tо

$$
\begin{aligned}
\mathscr{P}_{2}= & 2 p_{1}\left(\sqrt{\frac{a_{11}}{2}} q_{2} q_{t}-\sqrt{\frac{a_{22}}{2}} q_{1} q_{t}-\sqrt{\frac{a_{00}}{2}} q_{1} q_{2}\right)+p_{t} \frac{d t}{d \tau_{1-2}} \\
= & 2 q_{1} q_{2} p_{t}\left(\sqrt{\frac{a_{00}}{2}}-\frac{1}{2}\right) \\
& \quad-2 p_{t} q_{t}\left(\sqrt{\frac{a_{11}}{2}} q_{2}-\sqrt{\frac{a_{22}}{2}} q_{1}+\frac{q_{t}}{2}+\frac{1}{2}\left(q_{2}\left(Q_{t}\right)-q_{1}\left(Q_{t}\right)\right)\right) .
\end{aligned}
$$

Обозначим через $H_{t / 3}$ гамильтониан в переменных $p_{t}, q_{t}$ :

$$
\Omega_{\mathrm{PVI}}=d p_{t} \wedge d q_{t}-d H_{t / 3} \wedge d \tau_{1-2}
$$

Аналогично (92) получим, что гамильтониан $H_{t / 3}$ - многочлен от координат $X_{1}^{(t)}, X_{2}^{(t)}, X_{3}^{(t)}$ на квадрике $\mathscr{C}_{a_{00}^{\prime}}$ :

$$
X_{1}^{(t)} X_{2}^{(t)}+\left(X_{3}^{(t)}\right)^{2}=\left(\sqrt{\frac{a_{00}}{2}}-\frac{1}{2}\right)^{2}:=\frac{a_{00}^{\prime}}{2}, \quad a_{00}^{\prime}:=\left(\sqrt{a_{00}}-\sqrt{\frac{1}{2}}\right)^{2},
$$

где $X_{i}^{(t)}$ связаны с координатами $q_{t}, p_{t}$ стандартным образом:

$$
X_{1}^{(t)}=q_{t}, \quad X_{2}^{(t)}=-p_{t}\left(p_{t} q_{t}+2 \sqrt{\frac{a_{00}^{\prime}}{2}}\right), \quad X_{3}^{(t)}=p_{t} q_{t}+\sqrt{\frac{a_{00}^{\prime}}{2}} .
$$

Через $\sqrt{a_{00}^{\prime} / 2}$ обозначено одно из значений квадратного корня из $a_{00}^{\prime} / 2$ :

$$
\sqrt{\frac{a_{00}^{\prime}}{2}}:=\sqrt{\frac{a_{00}}{2}}-\frac{1}{2}
$$


Для гамильтониана $H_{t / 3}$ получаем выражение

$$
\begin{aligned}
H_{t / 3}=q_{1} & q_{2}\left(p_{t}\left(p_{t} q_{t}+2 \sqrt{\frac{a_{00}^{\prime}}{2}}\right)\right) \\
& -2 p_{t} q_{t}\left(\sqrt{\frac{a_{11}}{2}} q_{2}-\sqrt{\frac{a_{22}}{2}} q_{1}+\frac{q_{t}}{2}+\frac{q_{2}\left(Q_{t}\right)-q_{1}\left(Q_{t}\right)}{2}\right) \\
& +q_{t}\left(\frac{a_{33}}{2}-\left(\sqrt{\frac{a_{00}}{2}}+\sqrt{\frac{a_{11}}{2}}+\sqrt{\frac{a_{22}}{2}}\right)^{2}\right) \\
= & \left.X_{1}^{(t)}+t\right)\left(X_{1}^{(t)}+t-1\right) X_{2}^{(t)} \\
& -2\left(X_{3}^{(t)}-\sqrt{\frac{a_{00}^{\prime}}{2}}\right)\left(\left(\sqrt{\frac{a_{11}}{2}}+\sqrt{\frac{a_{22}}{2}}+\frac{1}{2}\right) X_{1}^{(t)}\right. \\
& +X_{1}^{(t)}\left(\frac{a_{33}}{2}-\left(\sqrt{\frac{a_{11}}{2}}(t-1)+\sqrt{\frac{a_{22}}{2}} t+t-\frac{1}{2}\right)\right.
\end{aligned}
$$

Обратим внимание на следующее важное обстоятельство.

УТВЕРЖДЕНИЕ 19. Точки квадрик $\mathscr{C}_{a_{00}^{\prime}} u \mathscr{C}_{a_{11}}$ отождествляются посредством равенств (93), т.е. это - симплектическая склейка $\mathscr{C}_{a_{11}} \uplus_{t}^{T} \mathscr{C}_{a_{00}^{\prime}}$.

Как мы знаем, при этой склейке к квадрике $\mathscr{C}_{a_{11}}\left(\right.$ к поверхности $\left.M_{a_{\bar{i}}}\right)$ над параболой " $q_{1}=-t=$ const" добавляется дивизор - линия $p_{1}=\infty, p_{1}^{*}=$ $1 / p_{1}=0$, попасть в точки которой можно, уходя на бесконечность

$$
p_{1} \rightarrow \infty, \quad q_{1} \rightarrow-t \quad \text { по } \mathscr{C}_{a_{11}} \sim U\left(-\sqrt{2 a_{00}} ; \sqrt{2 a_{22}}, 3\right) \subset M_{a_{\bar{i}}}
$$

специальным образом, а именно, так, чтобы величина $q_{t}^{*}:=-p_{1}\left(p_{1}\left(q_{1}+t\right)+\right.$ $\left.2 \sqrt{a_{00}^{\prime} / 2}\right)$ оставалась конечной. Обозначим этот дивизор $\mathscr{D}^{t}$.

ЗАмечАниЕ 25. Добавленный дивизор $\mathscr{D}^{t}$ соответствует вырождающимся наборам $A^{(\bar{i})}$ - обращающимся в бесконечность в момент $t$ решениям Пенлеве VI.

Определим многообразие $\mathscr{M}_{a_{\bar{i}}}^{T}$ - естественную область определения системы Пенлеве VI (расширенное фазовое пространство), добавив к $M_{a_{\bar{i}}}^{T}=M_{a_{\overline{i i}}} \times T$ линии $\mathscr{D}^{t}-$ над каждой $t \in T$ приклеим к $M_{a_{\bar{i}}}^{T}$ свою линию $\mathscr{D}^{t}$.

Построенное таким образом комплексно-трехмерное алгебраическое многообразие $\mathscr{M}_{a_{\bar{i}}}^{T}$ называется определяющим многообразием уравнения Пенлеве VI.

Объяснением к этому названию является теорема 15, которую мы докажем ниже.

На $\mathscr{M}_{a_{\overline{i i}}}^{T}$ естественным образом определено отображение

$$
Q_{-\sqrt{2 a_{00}}}^{\mathscr{M}}: \mathscr{M}_{a_{\bar{i} i}}^{T} \rightarrow L_{Q}^{(0)} \sim \mathbb{C P}^{1}
$$

Пересечение его слоя $\left(Q_{-\sqrt{2 a_{00}}}^{\mathscr{M}}\right)^{-1}\left(0: q_{1}: q_{2}: q_{3}\right)$ и симплектического листа $t=t_{0}=$ const в $\mathscr{M}_{a_{\bar{i}}}^{T}-$ это по построению комплексно-двумерное многообразие $M_{a_{\bar{i}}} \uplus_{t_{0}}^{T} \mathscr{C}_{a_{00}^{\prime}}$; оно называется поверхностью начальных данных уравнения Пенлеве VI, или поверхностью Окамото (см. [16], [8]). Обозначим ее $M_{a_{\bar{i}}, t}$. 
Рассмотрим квадрику $\mathscr{C}_{R_{0}^{2}}$ и набор квадрик $\mathscr{C}_{R_{i}^{2}}, i \in\{1, \ldots, k\}$, которые мы приклеим к $\mathscr{C}_{R_{0}^{2}}$ симплектическими склейками $\uplus_{t_{i}}^{T}$ или $\uplus_{C_{i}}^{Q}$ с какими-нибудь $t_{i}$, $C_{i} \in \mathbb{C}$. Обозначим получившееся, очевидно симплектическое, многообразие

$$
\mathscr{C}_{R_{0}^{2}}\left(\uplus^{(1)} \mathscr{C}_{R_{1}^{2}}\right)\left(\uplus^{(2)} \mathscr{C}_{R_{2}^{2}}\right) \cdots\left(\uplus^{(3)} \mathscr{C}_{R_{3}^{2}}\right),
$$

где $\uplus^{(i)}$ - это или $\uplus_{t_{i}}^{T}$, или $\uplus_{C_{i}}^{Q}$. Скобки $\left(\uplus^{(i)} \mathscr{C}_{R_{i}^{2}}\right)$ показывают, как $\mathscr{C}_{R_{i}^{2}}$ приклеиваются именно к $\mathscr{C}_{R_{0}^{2}}$. Конечно же, все остальные квадрики $\mathscr{C}_{R_{i}^{2}}$ и $\mathscr{C}_{R_{j}^{2}}$ тоже окажутся симплектически склеены друг с другом, но как именно - нам сейчас не важно.

УТВЕРЖДЕНИЕ 20. Поверхность Окамото $M_{a_{\bar{i}}, t}-$ симплектический лист системы Пенлеве VI может быть определен и так:

$$
M_{a_{\bar{i} i}, t}:=\mathscr{C}_{a_{11}}\left(\uplus_{1}^{T} \mathscr{C}_{a_{22}}\right)\left(\uplus_{\sqrt{a_{00} / 2}+\sqrt{a_{22} / 2}}^{Q} \mathscr{C}_{a_{33}}\right)\left(\uplus_{t}^{T} \mathscr{C}_{a_{00}^{\prime}}\right)
$$

ЗАмЕчАниЕ 26. Операции склейки $\uplus_{t}^{T}$ и $\uplus_{C}^{Q}$ квадрик определены нами в некоторых конкретных координатах $X_{1}^{(i)}, X_{2}^{(i)}, X_{3}^{(i)}$; мы указали способ построения таких координат (через построение стандартного сопутствующего базиса) на квадриках $\mathscr{C}_{a_{i i}}$, соответствующих вершинам $A^{(i)}, i=1,2,3$, а также на $\mathscr{C}_{a_{00}^{\prime}}$, соответствующей растущим решениям.

ЗАмечание 27 . Каждая из квадрик $\mathscr{C}_{a_{11}}, \mathscr{C}_{a_{22}}, \mathscr{C}_{a_{33}}, \mathscr{C}_{a_{00}^{\prime}}$ покрывается парой стандартных симплектических карт $U_{i}$ и $U_{i}^{*}$ с координатами $(p, q)$ и $\left(p^{*}, q^{*}\right)$, связанных между собой преобразованиями

$$
p^{*}=\frac{1}{p}, \quad q^{*}=-p\left(p q+2 \sqrt{\frac{a_{i i}^{\prime}}{2}}\right) ; \quad a_{i i}^{\prime}:=a_{i i}, \quad i=1,2,3,
$$

а между разными квадриками - преобразованиями склеек (88), (89). Таким образом получается традиционное определение (см. [16], [8]) многообразия начальных данных как абстрактного многообразия, определенного через функции переклейки.

Многообразие $\mathscr{M}_{a_{\bar{i}}}^{T}$ определяется как несвязное объединение всех $M_{a_{\bar{i} i}, t}$

$$
\mathscr{M}_{a_{\bar{i} i}}^{T}=\bigsqcup_{t \in T} M_{a_{\overline{i i}}, t} .
$$

На $\mathscr{M}_{a_{\bar{i}}}^{T}$ определена функция

$$
t: \mathscr{M}_{a_{\bar{i}}}^{T} \rightarrow \overline{\mathbb{C}} \backslash\{0,1, \infty\} .
$$

Прообразом точки $t$ является симплектический лист системы Пенлеве VI - поверхность Окамото $M_{a_{\bar{i}}, t}$. Гамильтоново поле направлений системы Пенлеве VI задает симплектические изоморфизмы (это мы доказывать не будем) между $M_{a_{\overline{i i}}, t}$ при разных $t$; при таком изоморфизме $q$-координата точки $(p(t), q(t))$, соответствующей какой-нибудь фиксированной точке $\left(q_{0}, p_{0}\right)$ на листе $t=t_{0}$, удовлетворяет уравнению Пенлеве VI. 
ОПРЕДЕЛЕНиЕ 4. Назовем поле направлений на (алгебраическом) многообразии $\mathscr{M}$ алгебраическим, если координаты прямых в касательном к $\mathscr{M}$ расслоении $T \mathscr{M}$, задающих это поле, алгебраически связаны с алгебраическими координатами на $T \mathscr{M}$.

На нечетномерном многообразии $M$ алгебраическое поле направлений может быть задано алгебраической внешней 2-формой $\Omega \subset \bigwedge^{2} T^{*} M-$ замкнутой и максимально не вырожденной. Заметим, что форма $\Omega$ обязана быть вырожденной (это - следствие нечетномерности $M$ ), но мы требуем вырожденности по линейному подпространству в $T M$ минимально возможной размерности (см. [32]) - по одномерному подпространству, т. е. по направлению. Чрезвычайно интересной представляется следующая теорема (см. [16], [8], [6]), которая позволяет свести некоторые (формально - все) вопросы исследования уравнения Пенлеве VI к исследованию алгебраического многообразия $\mathscr{M}_{a_{\bar{i}}}^{T}$.

Теорема 15. Поле направлений Пенлеве VI является единственным гладким алгебрачческим полем направлений на $\mathscr{M}_{a_{\bar{i} \mathbf{i}}}^{T}$.

Прежде чем переходить к доказательству теоремы, сформулируем (и докажем) несколько вспомогательных утверждений.

Прежде всего докажем, что $\mathscr{M}_{a_{\bar{i}}}^{T}$ содержит проективную кривую, т. е. существует гладкое непостоянное отображение некоторой компактной римановой поверхности в $\mathscr{M}_{a_{\bar{i}}}^{T}$. Для этого рассмотрим функцию $a_{0 t}$ (она же - гамильтониан $\left.-H_{1 / 3}\right)$ :

$$
a_{0 t}=-\left(a_{01}+t a_{03}\right) .
$$

УТВЕРЖДЕНИЕ 21. В той части $M_{a_{\bar{i}}, t}$, где координатами являются $p_{t}^{*}$ $u q_{t}^{*}$ :

$$
\begin{aligned}
p_{t}^{*} & :=\frac{1}{p_{t}}=-\frac{1}{p_{1}}, \\
q_{t}^{*} & :=-p_{t}\left(p_{t} q_{t}+2 \sqrt{\frac{a_{00}^{\prime}}{2}}\right)=\frac{1}{p_{1}}\left(\frac{q_{1}+t}{p_{1}}+2 \sqrt{\frac{a_{00}^{\prime}}{2}}\right), \\
q_{t} & =-p_{t}^{*}\left(p_{t}^{*} q_{t}^{*}+2 \sqrt{\frac{a_{00}^{\prime}}{2}}\right),
\end{aligned}
$$

функция $a_{0 t}$ как функция переменной $p_{t}^{*}$ (считая $q_{t}^{*}=$ const) имеет вдоль линии $\left(p_{t}^{*}=0, q_{t}^{*} \in \mathbb{C}\right)$ полюс (только) первого порядка с ненулевым вычетом.

ДокАзАТЕЛЬство. Выразим $a_{0 t}$ через $p_{t}^{*}$ и $q_{t}^{*}$, заменив $p_{1}$ и $q_{i}$ (формула (63)) на $p_{t}^{*}$ и $q_{t}^{*}$ :

$$
\begin{aligned}
-a_{0 t}= & \sqrt{2 a_{00}}(1-t) \beta_{1}+q_{t}\left(\frac{1}{p_{t}^{*}}\left(\beta_{1}+\sqrt{\frac{a_{11}}{2}}\right)\right. \\
& \left.-\left(\beta_{1}+\sqrt{\frac{a_{00}}{2}}+\sqrt{\frac{a_{22}}{2}}\right)^{2}+\frac{a_{33}}{2}\right)-\sqrt{2 a_{00}} t\left(\sqrt{\frac{a_{00}}{2}}+\sqrt{\frac{a_{22}}{2}}\right)
\end{aligned}
$$

где

$$
\beta_{1}=p_{1} q_{1}+\sqrt{\frac{a_{11}}{2}}=\frac{t}{p_{t}^{*}}+\sqrt{\frac{a_{11}}{2}}-\sqrt{2 a_{00}^{\prime}}-p_{t}^{*} q_{t}^{*} .
$$


Так как $q_{t}=-p_{t}^{*}\left(p_{t}^{*} q_{t}^{*}+\sqrt{2 a_{00}^{\prime}}\right)$ имеет корень первого порядка (по $\left.p_{t}^{*}\right)$, а $\beta_{1}-$ полюс первого порядка, то видно, что у $a_{0 t}$ действительно полюс только первого порядка, сосчитаем вычет:

$$
\sqrt{2 a_{00}}(1-t) t-\sqrt{2 a_{00}^{\prime}}\left(t-t^{2}\right)=t(t-1)\left(\sqrt{2 a_{00}^{\prime}}-\sqrt{2 a_{00}}\right)=-t(t-1) .
$$

Утверждение доказано, итак

$$
a_{0 t}=\frac{t(t-1)}{p_{t}^{*}}+\mathscr{P}\left(q_{t}^{*}, p_{t}^{*}\right)
$$

где $\mathscr{P}$ - многочлен.

УТВЕРЖДЕНИЕ 22. Функиия $f=f_{123}=\left\langle\left[A^{(1)}, A^{(2)}\right], A^{(3)}\right\rangle$ тоже имеет полюс на линии $p^{*}=0$. Это полюс третъего порядка.

ДокАзАтЕльство. Через $\beta_{i}, q_{i}, q_{i}^{\prime}$ функция $f$ записывается как определитель (49):

$$
f=q_{0}^{\prime}\left(q_{2} \beta_{1}-\sqrt{\frac{a_{22}}{2}} q_{1}\right)-\sqrt{\frac{a_{00}}{2}} q_{2} q_{1}^{\prime}=q_{2} \beta_{1} q_{0}^{\prime}+\mathscr{P},
$$

где $\mathscr{P}$ - многочлен от $p_{1}, q_{1}$, квадратичный по $p_{1}$;

$$
\begin{aligned}
& q_{1}^{\prime}=-p_{1}\left(p_{1} q_{1}+2 \sqrt{\frac{a_{11}}{2}}\right), \\
& q_{3}^{\prime}=\frac{a_{33}}{2}-\left(p_{1} q_{1}+\sqrt{\frac{a_{00}}{2}}+\sqrt{\frac{a_{11}}{2}}+\sqrt{\frac{a_{22}}{2}}\right)^{2}, \\
& q_{0}^{\prime}=-\left(q_{1}^{\prime}+q_{3}^{\prime}\right),
\end{aligned}
$$

где $q_{i}^{\prime}, i=0,1,3$, квадратичны по $p_{1}$. Вычислим главный член:

$$
\begin{aligned}
q_{2} \beta_{1} q_{0}^{\prime} & =\left(1+q_{1}\right) \beta_{1}\left(\frac{1}{p_{t}^{*}}\left(\beta_{1}+\sqrt{\frac{a_{11}}{2}}\right)-\left(\beta_{1}+\sqrt{\frac{a_{00}}{2}}+\sqrt{\frac{a_{22}}{2}}\right)^{2}+\frac{a_{33}}{2}\right) \\
& =\left(1+q_{1}\right) \frac{t}{p_{t}^{*}}\left(\frac{1}{p_{t}^{*}} \frac{t}{p_{t}^{*}}-\left(\frac{t}{p_{t}^{*}}\right)^{2}\right)\left(1+p_{t}^{*} \widetilde{\mathscr{P}}\right)=\frac{q_{2}}{\left(p_{t}^{*}\right)^{3}} t^{2}(t-1)\left(1+p_{t}^{*} \widetilde{\mathscr{P}}\right),
\end{aligned}
$$

где $\widetilde{\mathscr{P}}$ - многочлен от $q_{1}$ (или, что то же, - от $\left.q_{t}\right)$ и $p_{t}^{*}$.

Так как

$$
q_{2}=-\left(q_{1}+1\right)=t-1+q_{t}=t-1-p_{t}^{*}\left(p_{t}^{*} q_{t}^{*}+\sqrt{2 a_{00}^{\prime}}\right),
$$

то $f$ действительно имеет вид

$$
f=\frac{t^{2}(t-1)^{2}}{\left(p_{t}^{*}\right)^{3}}+\frac{1}{\left(p_{t}^{*}\right)^{2}} \widetilde{\widetilde{P}}\left(p_{t}^{*}, q_{t}^{*}\right), \quad \widetilde{\widetilde{P}}(\cdot, \cdot)-\text { многочлен. }
$$

Таким образом, у $f$ - полюс третьего порядка. 
СлЕДСТВИЕ 5. Для любого значения $q_{t}^{*}=c \in \mathbb{C}$ существуют такие $f_{1}=$ $f_{1}(c)$ и $f_{2}=f_{2}(c)$, что функиия

$$
\phi_{c}\left(p_{t}^{*}, q_{t}^{*}\right):=f-\frac{1}{t(t-1)} a_{0 t}^{3}+f_{2} a_{0 t}^{2}+f_{1} a_{0 t}
$$

не имеет особенности в точке $p_{t}^{*}=0, q_{t}^{*}=c$.

ДокАЗАТЕЛЬСтво. Действительно, вычет у $a_{0 t}$ ненулевой и можно подобрать такие $f_{2}$ и $f_{1}$, что $\phi_{c}$ не будет иметь особенности в этой точке.

Докажем, что на $M_{a_{\bar{i}}, t}$ не существует функций, отличных от константы. Для этого рассмотрим какое-нибудь $c \in \mathbb{C}$ и риманову поверхность $\mathscr{R}_{c}$ :

$$
f-\frac{1}{t(t-1)} a_{0 t}^{3}+f_{2}(c) a_{0 t}^{2}+f_{1}(c) a_{0 t}=\left.\phi_{c}\right|_{\substack{p_{t}^{*}=0 \\ q_{t}^{*}=c}}\left(p_{t}^{*}, q_{t}^{*}\right)=\mathrm{const},
$$

где $a_{0 t}=-\left(a_{01}+t a_{03}\right)$. Компактифицируем ее: $\overline{\mathscr{R}}_{c}:=\mathscr{R}_{c} \cup\{\infty\}$.

УтВЕРЖДЕНИЕ 23 (лЕММА). Множество $\{\infty\}$, добавленное $\kappa \mathscr{R}_{c}$ при компактификации, состоит из единственной точки, которая лежит на дивиsope $\mathscr{D}^{t}$.

ДокАЗАТЕЛЬСтво ЛЕммы. Рассмотрим $\overline{M^{f}}{ }_{a_{\bar{i}}}-$ компактификацию $M_{a_{\overline{i i}}}^{f}$, состоящую из $M_{a_{\bar{i}}}^{f}$ и добавленного на бесконечности дивизора $\{\infty\}_{M^{f}}$. Сечение поверхности $M_{a_{\bar{i}}}^{f}$ (формула (39))

$$
f^{2}=-2 \operatorname{det}\left|\begin{array}{ccc}
a_{11} & x & y \\
x & a_{22} & z \\
y & z & a_{33}
\end{array}\right|, \quad x+y+z=\frac{a_{00}-a_{11}-a_{22}-a_{33}}{2},
$$

плоскостью

$$
c_{1} x+c_{2} y+c_{3} z=0, \quad c_{1}: c_{2}: c_{3} \subset \mathbb{C P}^{2},
$$

$c_{1}+c_{2}+c_{3}=0,-$ это эллиптическая поверхность $f^{2}=\mathscr{P}_{3}(x)$ с единственной точкой (точкой ветвления) на бесконечности. Таким образом, каждому направлению $a_{01}: a_{02}: a_{03}, a_{0 i} \rightarrow \infty$ будет соответствовать единственная точка дивизора $\{\infty\}_{M^{f}}$. Поскольку $f^{2}=-2 a_{01} a_{02} a_{03}+\mathbf{O}\left(a_{0 i}\right)$, то вне направления $a_{01}: a_{02}: a_{03}=t_{1}: t_{2}: t_{3}$ разность

$$
f-\frac{1}{t(t-1)} a_{0 t}^{3}+f_{2} a_{0 t}^{2}+f_{1} a_{0 t}
$$

стремится к бесконечности и, следовательно, $\overline{\mathscr{R}}_{c}$ пересекает $\{\infty\}_{M^{f}}$ в единственной точке - там, где

$$
f \sim \frac{t^{2}(t-1)^{2}}{\left(p_{t}^{*}\right)^{3}}, \quad a_{01} \sim-\frac{t^{2}(t-1)}{\left(p_{t}^{*}\right)^{2}}, \quad a_{02} \sim \frac{t(t-1)^{2}}{\left(p_{t}^{*}\right)^{2}}, \quad a_{03} \sim \frac{t(t-1)^{2}}{\left(p_{t}^{*}\right)^{2}} .
$$

По построению $\phi_{c}\left(p_{t}, q_{t}^{*}\right)$ имеет там конечное значение, следовательно, риманова поверхность $\overline{\mathscr{R}}_{c}-$ линия уровня функции $\phi_{c}\left(p_{t}, q_{t}^{*}\right)$ при каждом $c \in \mathbb{C}$ проходит через точку дивизора $\mathscr{D}^{t}$, лемма доказана. 
Таким образом, компактная риманова поверхность $\bar{R}_{C}$ целиком содержится в $M_{a_{\bar{i}}, t}$ - единственная ее точка, не содержащаяся в $M_{a_{\bar{i}}}$, попала на $\mathscr{D}^{t}$. На любой компактной римановой поверхности нет непостоянных функций, следовательно, $\overline{\mathscr{R}}_{c}$ является линией уровня любой функции, определенной на $M_{a_{\bar{i}}, t}$ при любом значении $c$.

Все функции на $M_{a_{\bar{i}}}-$ многочлены от $a_{0 i}$ и $f$, следовательно, $H$ - функция на $M_{a_{\bar{i} \bar{i}}, t}$ имеет представление

$$
H=\mathscr{P}\left(a_{i 0}\right)+f \mathscr{P}^{\prime}\left(a_{i 0}\right),
$$

где $\mathscr{P}$ и $\mathscr{P}^{\prime}$ - какие-то многочлены от $a_{0 i}$. Функция $H$ должна быть постоянной на линии

$$
f-\frac{1}{t(t-1)} a_{0 t}^{3}+f_{2}(c) a_{0 t}^{2}+f_{1}(c) a_{0 t}=\phi_{c}(0, c)
$$

т. е. $H\left(a_{0 i}, f\right)=H_{c}=$ const, если $\left(f, a_{0 i}\right)$ с $\mathscr{R}_{c}$, откуда следует, что многочлен $H-H_{c}$ делится на многочлен

$$
\mathscr{P}_{\phi_{c}}:=f-\frac{a_{0 t}^{3}}{t(t-1)}+f_{2} a_{0 t}^{2}+f_{1} a_{0 t}-\phi_{c}(0, c),
$$

т. e.

$$
H=\left(f-\frac{a_{0 t}^{3}}{t(t-1)}+f_{2} a_{0 t}^{2}+f_{1} a_{0 t}-\phi_{c}(0, c)\right) H^{\prime}+H_{c},
$$

где $H^{\prime}$ - многочлен от $f, a_{0 i}$, уже меньшей чем $H$ степени, к которому можно применить те же рассуждения, так что

$$
H=\mathscr{P}\left(\phi_{c}\left(p_{t}^{*}, q_{t}^{*}\right)\right)
$$

- многочлен (одной переменной) от $f-a_{0 t}^{2} /(t(t-1))+f_{2} a_{0 t}^{2}+f_{1} a_{0 t}$. Подставив (95) в (96), убедимся, что $f_{1,2}=f_{1,2}(c)$ - нетривиальные функции $c \in \mathbb{C}$, так что $\phi_{c}\left(p_{t}^{*}, q_{t}^{*}\right)$ на дивизоре $p_{t}^{*}=0, q_{t}^{*} \in \mathbb{C}$ имеет конечное значение только в точке $q_{t}^{*}=c$; в остальных же точках дивизора (где $\left.q_{t}^{*} \neq c\right)$ функция $\phi_{c}\left(p_{t}^{*}, q_{t}^{*}\right)$ имеет полюс, значит, $\mathscr{P}=H=$ const.

Докажем теперь сформулированную выше теорему 15.

Предположим противное, т.е. что имеется еще одно поле направлений на $\mathscr{M}_{a_{\bar{i} i}}^{T} ;$ обозначим задающую его 2 -форму $\widetilde{\Omega}$ :

$$
\widetilde{\Omega} \in \bigwedge^{2} T^{*}\left(\mathscr{M}_{a_{\bar{i} \bar{i}}}^{T}\right),\left.\quad \widetilde{\Omega}\right|_{M_{a_{\bar{i}}}}=w .
$$

Составим разность $\Omega_{\mathrm{PVI}}-\widetilde{\Omega}-$ это 2-форма, обращающаяся в нуль на любой паре касательных к $M_{a_{\bar{i}}}$ векторов; значит, в каждой из карт $U_{i}, U_{i}^{*}$ (карты $U_{i}$ и $U_{i}^{*}$ определены в замечании 10, с. 80$)$ :

$$
\Omega_{\mathrm{PVI}}-\widetilde{\Omega}=d H_{i} \wedge d t
$$

причем в перекрытиях этих

$$
0=d H_{i} \wedge d t-d H_{j} \wedge d t=d\left(H_{i}-H_{j}\right) \wedge d t,
$$

следовательно, $H_{i}-H_{j}=C_{i j}(t)$, зафиксируем какое-нибудь $t$. 
Многообразие $M_{a_{\bar{i}},}$, покрыто окрестностями, где заданы функции, отличающиеся на константы; все окрестности пересекаются. Выберем одну из них там задана, например, $H_{1}$. Приравняем ей другие, добавив к $H_{j}$ константу $c_{1 j}=c_{1 j}(t)$.

Мы получили глобально на $M_{a_{\bar{i} i}, t}$ заданную функцию. Она обязана быть константой.

\section{8. Заключение}

В заключение хочу выразить благодарность всем, кто содействовал появлению данного текста.

Прежде всего это Л.А. Бордаг - за сочувствие и поддержку, без нее автор просто не имел бы возможности сделать эту работу. Очень благодарен С. Ю. Славянову. Беседы с ним помогли осознать проблему; отдельное ему спасибо за регулярную материальную (не финансовую!) поддержку и помощь советы, редактирование рукописи.

В вопросах алгебраической геометрии помощниками и наставниками были Г. Ю. Панина (Шахбазян), И. А. Панин и А. Л. Смирнов. В понимании теории гамильтоновых систем на алгебрах Ли большую помощью оказал М. А. Семенов-Тянь-Шанский. Благодарен И. М. Кричеверу за рецензирование рукописи и ряд важных замечаний.

К сожалению, работа продолжалась столь долго, что некоторым уже не суждено прочитать эти строки - я имею в виду А. А. Болибруха и А. Н. Тюрина, чье участие и помощь были неоценимы.

И наконец последнее - за годы работы над этой темой были сотни бесед с десятками людей, так что автор надеется на прощение со стороны невольно обиженных - всех упомянуть невозможно, где-то нужно было поставить точку.

\section{Список литературы}

[1] K. Iwasaki, H. Kimura, S. Shimomura, M. Yoshida, "From Gauss to Painlevé. A modern theory of special functions", Aspects Math., E16, Friedr. Vieweg \& Sohn, Braunschweig, 1991.

[2] K. Okamoto, "Studies in the Painlevé equations. I. Sixth Painlevé equation $\mathrm{P}_{\mathrm{VI}}$ ", Ann. Mat. Pura Appl. (4), 146:1 (1986), 337-381.

[3] А. Р. Итс, А. А. Капаев, В. Ю. Новокшенов, А. С. Фокас, Трансценденты Пенлеве. Метод задачи Римана, Ин-т компьютер. исслед., М.-Ижевск, 2005; англ. пер.: A. S. Fokas, A. R. Its, A. A. Kapaev, V. Yu. Novokshenov, Painlevé transcendents. The Riemann-Hilbert approach, Math. Surveys Monogr., 128, Amer. Math. Soc., Providence, RI, 2006.

[4] S. Yu. Slavyanov, W. Lay, "Special functions. A unified theory based on singularities", Oxford Math. Monogr., Oxford Univ. Press, Oxford, 2000; пер. с англ.: С. Ю. Славянов, В. Лэй, Специальные функиии: Единая теория, основанная на анализе особенностей, Невский Диалект, СПб., 2002.

[5] Yu. I. Manin, "Sixth Painlevé equation, universal elliptic curve, and mirror of $\mathbf{P}^{2}$ ", Geometry of differential equations, Amer. Math. Soc. Transl. Ser. 2, 186, Amer. Math. Soc., Providence, RI, 1998, 131-151. 
[6] D. Arinkin, S. Lysenko, "Isomorphisms between moduli spaces of SL(2)-bundles with connections on $\mathbf{P}^{1} \backslash\left\{x_{1}, \ldots, x_{4}\right\}$ ", Math. Res. Lett., 4:2-3 (1997), 181-190.

[7] N. Hitchin, "Geometrical aspects of Schlesinger's equation", J. Geom. Phys., 23:3-4 (1997), 287-300.

[8] T. Shioda, K. Takano, "On some Hamiltonian structures of Painlevé systems. I", Funkcial. Ekvac., 40:2 (1997), 271-291.

[9] M. Jimbo, T. Miwa, K. Ueno, "Monodromy preserving deformation of linear ordinary differential equations with rational coefficients. I. General theory and $\tau$-function", Phys. D, 2:2 (1981), 306-352.

[10] M. Jimbo, T. Miwa, "Monodromy preserving deformation of linear ordinary differential equations with rational coefficients. II", Phys. D, 2:3, 407-448; "Monodromy preserving deformation of linear ordinary differential equations with rational coefficients. III", Phys. D, 4:1 (1981), 26-46.

[11] A. V. Kitaev, D. A. Korotkin, "On solutions of the Schlesinger equations in terms of

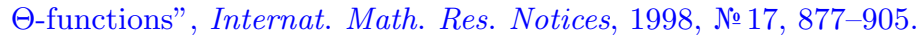

[12] B. Dubrovin, M. Mazzocco, "Canonical structure and symmetries of the Schlesinger equations", Comm. Math. Phys., 271:2 (2007), 287-373; arXiv:math/0311261v4, 2003.

[13] А.А. Болибрух, 21-я проблема Гильберта для фуксовых линейных систем, Тр. МИАН, 206, Наука, М., 1994; англ. пер.: A. A. Bolibrukh, Hilbert's twenty-first problem for linear Fuchsian systems, Proc. Steklov Inst. Math., 5, 1995.

[14] А.А. Болибрух, Фуксовы дифференииальные уравнения и голоморфные расслоения, МЦНМО, М., 2000.

[15] Б. А. Дубровин, С. П. Новиков, А. Т. Фоменко, Современная геометрия: Методъ и приложения, Наука, М., 1979; англ. пер.: В. A. Dubrovin, A. T. Fomenko, S. P. Novikov, Modern geometry - methods and applications. Part I: The geometry of surfaces, transformation groups, and fields, Grad. Texts in Math., 93, Springer-Verlag, New York, 1984; Modern geometry - methods and applications. Part II: The geometry and topology of manifolds, Grad. Texts in Math., 104, Springer-Verlag, New York, 1985.

[16] K. Okamoto, "Sur les feuilletages associés aux équations du second ordre à points critiques fixes de P. Painlevé", Japan. J. Math. (N. S.), 5 (1979), 1-79.

[17] H. Flashka, D. W. McLaughlin, "Canonically conjugate variables for the Kortewegde Vries equation and the Toda lattice with periodic boundary conditions", Progr. Theoret. Phys., 55:2 (1976), 438-456.

[18] А.П. Веселов, С.П. Новиков, "О скобках Пуассона, согласованных с алгебраической геометрией и динамикой Кортевега-де Фриза на множестве конечнозонных потенциалов", Докл. АН CCCP, 266:3 (1982), 533-537; англ. пер.: S. P. Novikov, A. P. Veselov, "On Poisson brackets compatible with algebraic geometry and Korteweg-de Vries dynamics on the set of finite-zone potentials", Soviet Math. Dokl., 26 (1982), 357-362.

[19] I. M. Krichever, D. H. Phong, "Symplectic forms in the theory of solitons", Surveys in differential geometry: integral systems, Surv. Differ. Geom., 4, Int. Press, Boston, MA, 1998, 239-313.

[20] I. Krichever, "Vector bundles and Lax equations on algebraic curves", Comm. Math. Phys., 229:2 (2002), 229-269.

[21] И. М. Кричевер, “Аналог формулы Даламбера для уравнений главного кирального поля и уравнения sine-Gordon", Докл. АН CСCP, 253:2 (1980), 288-292; англ. пер.: I. M. Krichever, "An analogue of the d'Alembert formula for the equations of a principal chiral field and the sine-Gordon equation", Soviet Math. Dokl., 22:1 (1980), 79-84. 
[22] D. V. Anosov, A. A. Bolibruch, "The Riemann-Hilbert problem", Aspects Math., E22, Vieweg, Braunschweig, 1994.

[23] A. A. Bolibruch, "On isomonodromic deformations of Fuchsian systems", J. Dynam. Control Systems, 3:4 (1997), 589-604.

[24] М.В. Бабич, "О координатах на фазовых пространствах системы уравнений Шлезингера и системы уравнений Гарнье-Пенлеве 6", Докл. РАН, 412:4 (2007), 439-443; англ. пер.: M. V. Babich, "About coordinates on the phase spaces of the Schlesinger system and the Garnier-Painlevé 6 system", Doklady Math., 75:1 (2007), $71-75$.

[25] М.В. Бабич, Уравнения Шлезингера и уравнения Гарнъе-Пенлеве 6, геометрия перехода, Препринт № 06.2006, ПОМИ, Спб., 2006.

[26] M. V. Babich, About coordinates on the phase-spaces of Schlesinger system $(n+1$ matrices, sl(2,C)-case) and Garnier-Painlevé 6 system, arXiv: math/0605544, 2006.

[27] И.Р. Шафаревич, Основы алгебраической геометрии. Т. 1. Алгебраические многообразия в проективном пространстве, Наука, М., 1988; англ. пер.: I. R. Shafarevich, Basic algebraic geometry. I: Varieties in projective space, Springer-Verlag, Berlin, 1994.

[28] L. Fuchs, "Uber Differenzialgleichungen, deren Integrale feste Verzweigunspunkte ezitzen", Sitzungsber. Konigl. Preuss. Akad. Wiss. Berlin, 32 (1884), 699-710.

[29] A. H. M. Levelt, "Hypergeometric functions. I", Nederl. Akad. Wetensch. Proc. Ser. A 64, 23 (1961), 361-372; "Hypergeometric functions. II", 373-385; "Hypergeometric functions. III", 386-396; "Hypergeometric functions. IV", 397-403.

[30] B. Dubrovin, "Geometry of 2D topological field theories", Integrable systems and quantum groups (Montecatini Terme, 1993), Lecture Notes in Math., 1620, Springer, Berlin, 1996, 120-348.

[31] L. Schlesinger, "Über eine Klasse von Differentialsystemen beliebiger Ordnung mit festen kritischen Punkten", J. Reine Math., 141 (1912), 96-145.

[32] В. И. Арнольд, Математические методы классической механики, 3-е изд., Наука, М., 1989; англ. пер.: V. I. Arnol'd, Mathematical methods of classical mechanics, Second edition, Grad. Texts in Math., 60, Springer-Verlag, New York, 1989.

[33] А.Г. Рейман, М.А. Семенов-Тянь-Шанский, Интегрируемые системъ. Tеоретико-групповой подход, Современная математика, Ин-т компьютер. исслед., М.-Ижевск, 2003.

[34] J. Marsden, A. Weinstein, "Reduction of symplectic manifold with symmetry", Rep. Math. Phys., 5:1 (1974), 121-130.

[35] В.В. Трофимов, Введение в геометрию многообразий с симметриями, Изд-во МГУ, М., 1989; англ. пер.: V. V. Trofimov, Introduction to geometry of manifolds with symmetry, Math. Appl., 270, Kluwer Acad. Publ., Dordrecht, 1994.

[36] М. М. Постников, Группы Ли и алгебры Ли, Лекции по геометрии, семестр V, Наука, М., 1982; англ. пер.: М. Postnikov, Lie groups and algebras, Lectures in geometry. Semester V, Mir, Moscow, 1986.

[37] M. Audin, "Lectures on gauge theory and integrable systems", Gauge theory and symplectic geometry (Montreal, PQ, 1995), NATO Adv. Sci. Inst. Ser. C Math. Phys. Sci., 488, Kluwer Acad. Publ., Dordrecht, 1997, 1-48.

[38] M. Kapovich, M.J. Millson, "The symplectic geometry of polygons in Euclidean space", J. Differential Geom., 44:3 (1996), 479-513.

[39] С. Ю. Славянов, "Изомонодромные деформации уравнений класса Гойна и уравнения Пенлеве", ТМФ, 123:3 (2000), 395-406; англ. пер.: S. Yu. Slavyanov, "Isomonodromic deformations of Heun and Painlevé equations", Theoret. and Math. Phys., 123:3 (2000), 744-753. 
[40] С.Ю. Славянов, Ф.Р. Вукайлович, "Изомонодромные деформации и 'антиквантование' для простейших ОДУ”, ТМФ, 150:1 (2007), 143-151; англ. пер.: S. Yu. Slavyanov, F.R. Vukajlović, "Isomonodromic deformations and 'antiquantization' for the simplest ordinary differential equations", Theoret. and Math. Phys., 150:1 (2007), 123-131.

[41] Н. И. Ахиезер, Элементы теории эллиптических функиий, 2-е изд., Физико-математическая библиотека инженера, 2, Наука, М., 1970; англ. пер.: N. I. Akhiezer, Elements of the theory of elliptic functions, Transl. Math. Monogr., 79, Amer. Math. Soc., Providence, RI, 1990.

[42] M. V. Babich, L. A. Bordag, "Projective differential geometrical structure of the Painlevé equations", J. Differential Equations, 157:2 (1999), 452-485.

M. В. Бабич (M. V. Babich)

Поступила в редакцию

Санкт-Петербургское отделение

20.10 .2008

Математического института им. В. А. Стеклова РАН

E-mail: mbabich@pdmi.ras.ru 